\title{
INTERACTION OF THE LONG-PERIOD ULF WAVES AND CHARGED PARTICLE IN THE MAGNETOSPHERE: THEORY AND OBSERVATIONS (OVERVIEW)
}

\section{D.Yu. Klimushkin}

Institute of Solar-Terrestrial Physics SB RAS,

Irkutsk,Russia,klimush@iszf.irk.ru

\author{
P.N. Mager \\ Institute of Solar-Terrestrial Physics SB RAS, \\ Irkutsk,Russia,p.mager@iszf.irk.ru
}

\author{
M.A. Chelpanov \\ Institute of Solar-Terrestrial Physics SB RAS, \\ Irkutsk, Russia,max_chel@isz.irk.ru \\ D.V. Kostarev \\ Institute of Solar-Terrestrial Physics SB RAS, \\ Irkutsk,Russia,kostarev@iszf.irk.ru
}

\begin{abstract}
The paper reviews the current state of the problem of interaction between long-period ultra-lowfrequency (ULF) waves and high-energy particles. We consider elements of the theory of energy exchange between waves and particles, particle transport across magnetic shells under the influence of the electromagnetic field of a wave, the acceleration of radiation belt particles by both resonant and non-resonant mechanisms. We examine the mechanisms of generation of azimuthallysmall-scale ULF waves due to instabilities arising from the wave-particle resonance. The cases of Alfvén, driftcompressional, and drift-mirror waves are analyzed. It is noted that due to the lack of a detailed theory of driftmirror modes, the possibility of their existence in the
\end{abstract}

magnetosphere cannot be taken as a proven fact. We summarize experimental data on the poloidal and compression ULF waves generated by unstable populations of high-energy particles. We investigate the mechanisms of modulation of energetic particle fluxes by ULF waves and possible observational manifestations of such modulation. Methods of studying the structure of waves across magnetic shells by recording fluxes of resonant particles with a finite Larmor radius are discussed.

Keywords: ULF waves, wave-particle interaction, radiation belts, plasma instabilities.

\section{Contents}

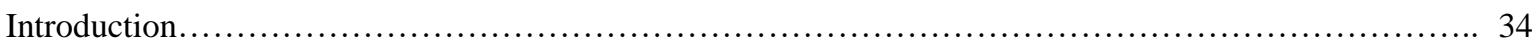

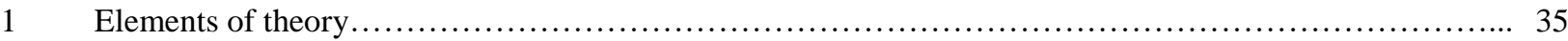

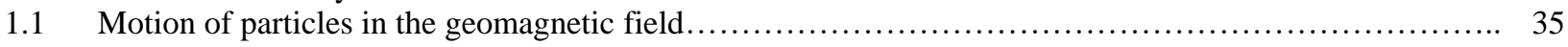

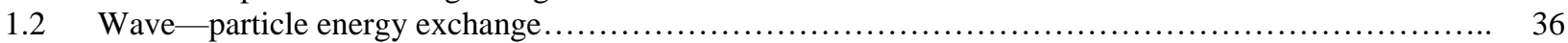

1.3 Particle transport across magnetic shells...................................................... 38

1.4 Dynamics of a near-equatorial particle in the field of an Alfvén wave............................... 39

2 The role of ULF waves in the acceleration of radiation belt particles................................ 39

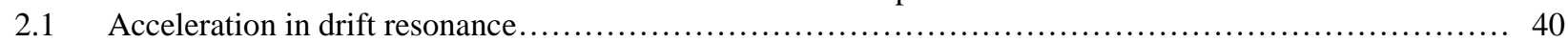

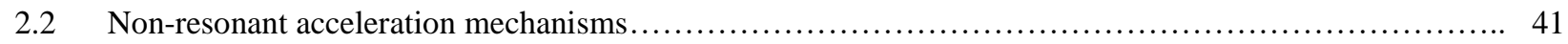

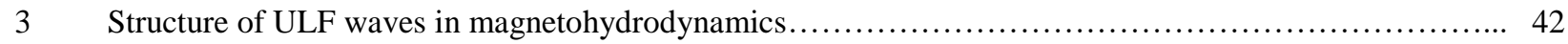

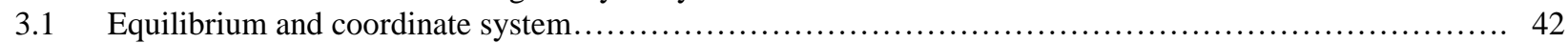

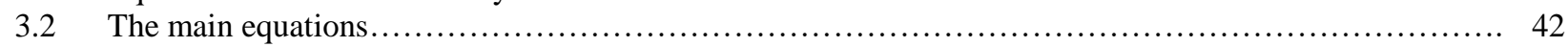

3.3 Structure of Alfvén waves in the region of monotonic variation of toroidal and poloidal frequencies...... 44

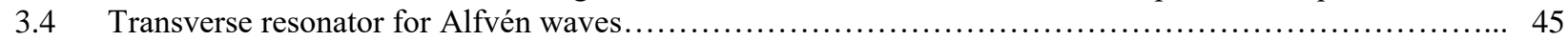

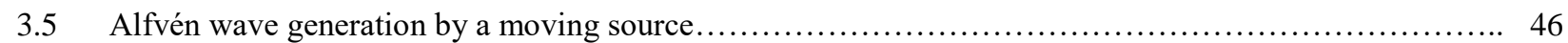

4 ULF waves in kinetics. ULF wave increase due to resonant interaction with energetic particles.......... 46

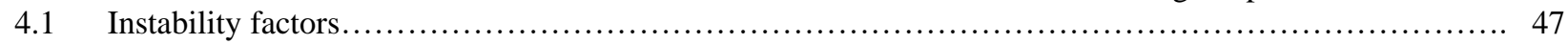

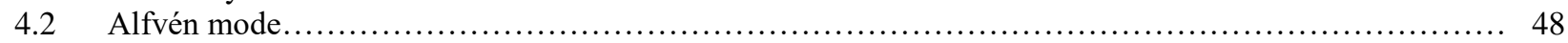

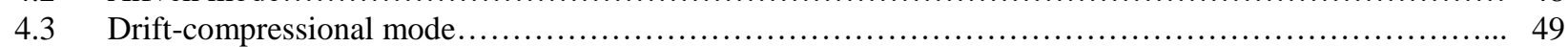

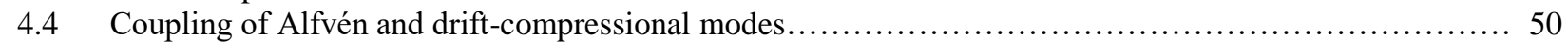

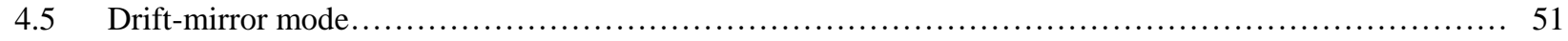

5 Experimental data on small-scale azimuthal wave generation by instabilities....................... 52

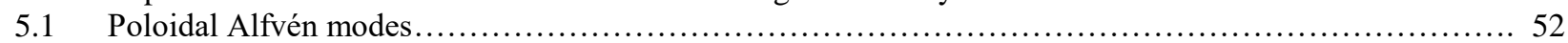

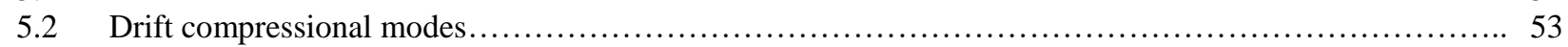

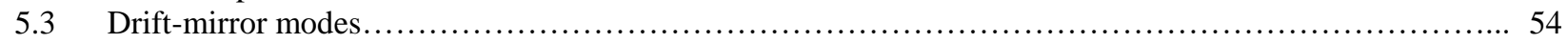

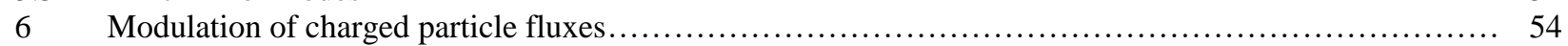

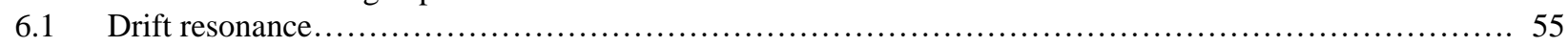

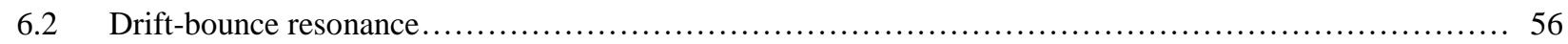

6.3 Finite gyroradius effect and determination of the wave vector transverse component $\ldots \ldots \ldots \ldots \ldots \ldots \ldots .57$

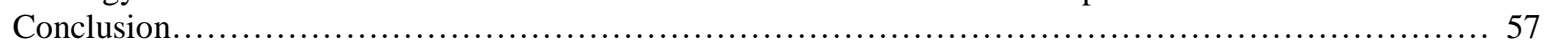




\section{INTRODUCTION}

Ultra-low-frequency (ULF) waves, or geomagnetic pulsations, are oscillations in Earth's magnetosphere with frequencies of order or lower than the proton gyrofrequency (from millihertz to several hertz). The ULF waves are divided into two large branches: short-period (Pcl-2, Pil; frequencies from 5 to $0.1 \mathrm{~Hz}$ ) and long-period (Pc3-5. Pi2-3; from $0.1 \mathrm{~Hz}$ to $1 \mathrm{MHz}$ ). In this review, we deal with the long-period ULF waves whose characteristic feature is a wavelength comparable in order of magnitude to the length of the magnetic field line. Below this frequency range are magnetic bays - aperiodic magnetic disturbances accompanying substorms.

The long-period ULF waves are divided into three types from an observational point of view: toroidal, poloidal, and compressional. The toroidal ULF waves are geomagnetic field line oscillations in the azimuthal direction (Figure 1, left panel). In this case, the electric field oscillates mainly in the radial direction. In the poloidal ULF waves, geomagnetic field lines generally oscillate in the radial (meridional) direction (Figure 1, right panel). In this case, the electric field fluctuations occur along the azimuth. The magnetic field magnitude practically does not change in toroidal and poloidal waves. On the contrary, in compressional waves the magnetic field absolute value oscillates. This means that there is a significant parallel component of the magnetic field disturbance.

ULF waves are also divided into azimuthally largescale and small-scale (azimuthal wave numbers $m \sim 1$ and $m>>1$ respectively). Toroidal waves are mainly azimuthally large-scale; poloidal ones, azimuthally small-scale. ULF waves also have different generation mechanisms: the sources of large-scale waves are associated with the solar wind, the sources of smallscale waves are located inside the magnetosphere. Azimuthally mean-scale oscillations are also often introduced $(m \simeq 10)$ recently [Yeoman et al., 2010; Hao et al., 2014; Mager et al., 2019]. This case has been relatively little studied so far. The azimuthal wave number sign is also of great importance. Waves with $m>0$ propagate in the magnetosphere to the east, in the electron drift direction; waves with $m<0$, to the west, in the proton drift direction.

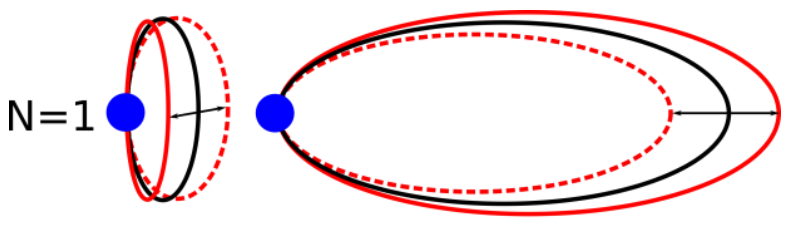

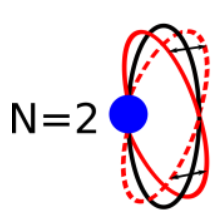

Toroidal

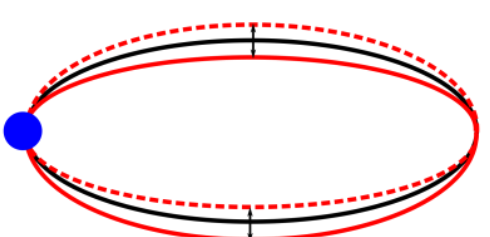

Poloidal
Figure 1. Toroidal and poloidal oscillations of field lines. The main and second harmonics are shown $(N=1$ and $N=2$ respectively)
Approximately $90 \%$ of azimuthally small-scale waves have negative azimuthal wave numbers [Chelpanov et al., 2019].

From the physical point of view, both toroidal and poloidal oscillations are identified with Alfvén waves standing along the magnetic field line between magnetically conjugated ionosphere points [Dungey, 1954; Radoski, 1967]. Among the compressional waves, there can be both fast and slow magnetosonic MHD waves, as well as the modes of the kinetic nature, like drift-compressional or drift-mirror modes.

Already in the early 1960s, an opinion was stated that ULF waves can play a crucial role in the dynamics of high-energy particles in the magnetosphere (radiation belt and ring current particles) [Dungey, 1964]. The waves can accelerate particles, transport them across magnetic shells, transfer them to a loss cone, and thereby contribute to their disappearance from the magnetosphere. In the late $60 \mathrm{~s}$ - early $70 \mathrm{~s}$, the possibility of a reverse influence was discovered: highenergy particle populations can generate waves through various plasma instabilities [Southwood et al., 1969; Hasegawa, 1969; Mikhailovskii and Pokhotelov, 1975]. It is often assumed that plasma instabilities are responsible for the generation of azimuthally smallscale ULF waves.

The interaction between ULF waves and particles was the subject of several large reviews, which dealt with both the general state of the problem [Dungey, 1964; Southwood, 1980; Tamao, 1984a; Zong et al., 2017] and the role of ULF waves in the acceleration of radiation belt particles [Elkington, 2006; Shprits et al., 2008; Mann et al., 2012; Elkington and Sarris, 2016; Potapov, 2017; Lejosne and Kollmann, 2020]. The overview of the role of interaction with particles in the generation of higher frequency waves (whistlers, ioncyclotron waves) is presented in [Tsurutani and Lakhina, 1997; Demekhov, 2007; Trakhtengerts and Rycroft, 2008]. The influence of nonlinear effects, including those associated with the wave-particle interaction, on short-period ULF wave propagation has been examined in [Guglielmi and Potapov, 2021].

In this review, we delve into the current state of the problem of the interaction between long-period ULF waves and particles. We address both the problem of particle acceleration by waves and the problem of wave generation by particles. The material is presented in a form accessible to the early career researcher.

The structure of the review is as follows. Section 1 addresses general problems of the wave-particle interaction theory: growth of drift-bounce and drift resonances, movement of a particle transversely to magnetic shells under the action of the wave's electromagnetic field, the dynamics of near-equatorial particles. Section 2 discusses the main ideas that explain the role of ULF waves in accelerating radiation belt particles, including "killer electrons" with relativistic energies. Section 3 describes the ULF wave theory's main results, especially for waves with large azimuthal wave numbers, which are generated due to interaction with particles. The theory of long-period ULF wave 
generation due to plasma instabilities associated with energy transfer from high-energy particles is described in Section 4. Section 5 deals with azimuthally smallscale waves, generated by particles, from an experimental point of view. Theoretical and experimental problems of particle fluxes modulation by ULF waves are explored in Section 6. In conclusion, the main results of our review are outlined.

\section{ELEMENTS OF THEORY}

\section{field}

\subsection{Motion of particles in the geomagnetic}

At first, it makes sense to review the basics of the theory of charged particle motion in Earth's magnetosphere [Zelenyi et al., 2010; Trakhtengerts and Rycroft, 2008]. Motion of a particle in the inhomogeneous magnetic field can be decomposed into two components: gyration about a magnetic field line and guiding center motion, i.e. the motion of the center of rotation (Figure 2). The guiding center approximation applies if (1) a characteristic spatial scale of plasma or magnetic field inhomogeneity is considerably larger than particle's Larmor radius and (2) a characteristic time scale of changes in plasma and field parameters is longer than the period of rotation of a particle around a field line (gyroperiod). A gyration about a field line is a circular motion with a gyrofrequency (cyclotron frequency)

$$
\omega_{\mathrm{c}}=\frac{q B}{M c} .
$$

Here, $q$ and $M$ are particle charge and mass, $B$ is the magnetic field, $c$ is the speed of light. In the projection onto a plane perpendicular to a field line, a trajectory of a particle is a circle with a Larmor radius:

$$
r_{\mathrm{L}}=\frac{M c v_{\perp}}{q B}
$$

where $v_{\perp}$ is a component of the particle velocity normal to the field line. The directions of electron and ion cyclotron rotation are opposite.

The guiding center motion equation is:

$$
\frac{d \vec{V}}{d t}=\frac{q}{M}\left\{\vec{E}+\frac{1}{c} \vec{V} \times \vec{B}\right\}-\frac{\mu}{M} \nabla B
$$

[Northrop, 1963]. The guiding center velocity $\vec{V}$ consists of two components: oscillations along a field line and a drift in the azimuthal direction, $\vec{V}=\vec{v}_{\|}+\vec{u}_{\mathrm{d}}$.

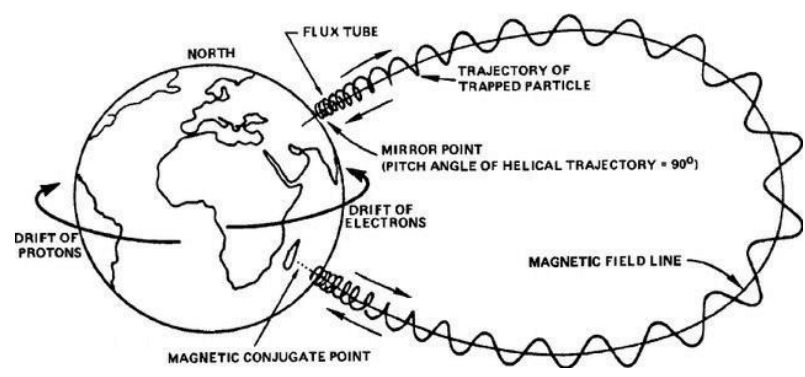

Figure 2. Motion of particles in the geomagnetic field
While a guiding center moves in a stationary-magnetic field, four parameters remain constant: kinetic particle energy $\varepsilon=M v^{2} / 2$ (hereafter, the particle energy denotes its kinetic energy) and three adiabatic invariants. The first adiabatic invariant is the magnetic moment of a particle

$$
\mu=\frac{M v_{\perp}^{2}}{2 B} \text {. }
$$

The expression for the longitudinal velocity of particle follows from the conservation of energy and the magnetic moment:

$$
v_{\|}=\sqrt{\frac{2}{M}(\varepsilon-\mu B)} .
$$

The second (longitudinal) adiabatic invariant is defined by the integral

$$
J=2 M \int_{l_{1}}^{l_{2}} v_{\|} d l .
$$

The total magnetic flux enclosed by a drift trajectory of a particle is the third adiabatic invariant:

$$
\Psi=\int \vec{B} \cdot d \vec{S},
$$

where $d S$ is a differential of an area within a particle's drift trajectory.

The motion along a field line is oscillatory. Its period (bounce period) is

$$
\tau_{\mathrm{b}}=2 \int_{l_{1}}^{l_{2}} \frac{d l}{v_{\|}} .
$$

Here, $l$ is the trajectory length along a field line, $l_{1,2}$ are coordinates of its turning points (mirror points). The frequency of oscillations along a field line (bounce frequency) is defined by the expression

$$
\omega_{\mathrm{b}}=\frac{2 \pi}{\tau_{\mathrm{b}}}
$$

In a dipole magnetic field, the bounce period is calculated using the approximate relation

$$
\tau_{\mathrm{b}} \approx \frac{4 L}{v}\left(1.30-0.56 \sin \alpha_{0}\right),
$$

[Hamlin et al., 1961], where $v$ is the absolute value of the particle velocity, $L$ is the distance from Earth's center to a point where a field line crosses the geomagnetic equator (measured in Earth's radii, $R_{\mathrm{E}}$ this is the McIlwain parameter), $\alpha_{0}$ is the equatorial pitch angle of a particle (the angle between the velocity and the magnetic field at the equator).

The azimuthal drift is caused by two reasons: inhomogeneity of the magnetic field and field line curvature. The total drift velocity is

$$
u_{\mathrm{d}}=\frac{1}{\omega_{\mathrm{c}}}\left(\frac{\nabla_{\perp} B}{2 B} v_{\perp}^{2}-\frac{v_{\|}^{2}}{R}\right),
$$

where $R$ is the local radius of field line curvature, $\nabla_{\perp}$ denotes the differential operator $\nabla$ projection onto a line perpendicular to the magnetic field $\left(\nabla_{\perp} B\right.$ is scalar; in the magnetosphere typically $\nabla_{\perp} B<0$ ). Positive ions drift 
westward, and electrons drift eastward (clockwise and counterclockwise, respectively, viewed from the geomagnetic north pole). The electric field in the magnetosphere also induces drift motion:

$$
\vec{u}_{E}=c \frac{\vec{E} \times \vec{B}}{B^{2}},
$$

However, it is insignificant for high-energy particles, and we will not take it into account (although it will play an essential role in the study of the particle dynamics in the electromagnetic wave field).

The angular frequency of the magnetic drift is defined by the relation

$$
\omega_{\mathrm{d}}=\frac{u_{\mathrm{d}}}{r \cos \lambda}
$$

where $r$ is the distance from Earth's center, $\lambda$ is the geomagnetic latitude measured from the geomagnetic equator. In the theory, the drift frequency averaged over the bounce period is a highly significant parameter:

$$
\bar{\omega}_{\mathrm{d}}=\frac{1}{\tau_{\mathrm{b}}} \int_{l_{1}}^{l_{2}} \omega_{\mathrm{d}} \frac{d l}{v_{\|}} .
$$

In a dipole magnetic field, this parameter is calculated using the approximate relation

$$
\bar{\omega}_{\mathrm{d}} \approx-\frac{3 v^{2}}{\omega_{\mathrm{c}, \mathrm{eq}} L^{2}}\left(0.35+0.15 \sin \alpha_{0}\right),
$$

where $\omega_{\mathrm{c}, \mathrm{eq}}$ is the cyclotron frequency at the equator at a given magnetic shell [Hamlin et al., 1961]. The electron drift direction is chosen as the positive drift direction (to the east); for electrons $\omega_{\mathrm{c}}<0$.

\subsection{Wave-particle energy exchange}

Due to the interaction with the electromagnetic field, the instantaneous particle energy changes over time as

$$
\frac{d \varepsilon}{d t}=q \vec{v} \cdot \vec{E}
$$

Frequencies of ULF waves are much lower than the gyrofrequency of protons. Therefore, relation (16) must be averaged over the gyroperiod. The resulting relation is [Northrop, 1963]:

$$
\frac{d\langle\varepsilon\rangle}{d t}=q v_{\|} E_{\|}+q \vec{u}_{\mathrm{d}} \cdot \vec{E}_{\perp}+\mu \frac{\partial B}{\partial t} .
$$

Here, the angle brackets denote averaging over the gyroperiod. Hereafter, we will omit the angle brackets for this parameter, i.e. $\varepsilon$ denotes particle energy averaged over the gyroperiod.

The absolute value of the magnetic field is $B=\sqrt{\left(\vec{B}_{0}+\vec{B}_{1}\right)^{2}}$, (where $B_{0}$ is the ambient magnetic field, and $B_{1}$ is the magnetic field of a wave (hereafter, the " 1 " index is omitted for wave parameters). Linearizing this relation with $B_{1} \ll<B_{0}$ reveals $B \simeq \sqrt{B_{0}^{2}+2 \vec{B}_{0} \cdot \vec{B}_{1}} \simeq B_{0}+B_{\|}$, where the second term means the longitudinal (compressional) component of the wave magnetic field. Assuming the external field to be stationary, (17) can be transformed in:

$$
\frac{d \varepsilon}{d t}=q v_{\|} E_{\|}+q u_{\mathrm{d}} E_{\mathrm{a}}+\mu \frac{\partial B_{\|}}{\partial t} .
$$

The second term implies that the drift occurs in the azimuthal direction such that of the transverse electric field components, only the azimuthal one $E_{\mathrm{a}}$ contributes to the energy change.

Since a particle moves in the alternating electromagnetic field of a wave, it gains energy on a part of its trajectory and loses it on another part. We will consider monochromatic waves for which the following relations apply:

$$
E, B \propto \exp i(m \varphi-\omega t),
$$

where $\omega$ is the wave frequency, $m$ is the azimuthal wave number, $\varphi$ is the azimuthal angle. A continuous increase or decrease in energy is only possible when a moving particle constantly "sees" field of the same sign. In the case of long-period ULF waves, with $\omega \ll \omega_{c}$, energy exchange is defined by relation (18), and the driftbounce resonance condition is required [Dungey, 1964]:

$$
\omega-m \bar{\omega}_{\mathrm{d}}-K \omega_{\mathrm{b}}=0
$$

where $K$ is an integer. The $K=0$ condition results in the drift resonance:

$$
\omega-m \bar{\omega}_{\mathrm{d}}=0 .
$$

Note that at higher frequencies the wave-particle resonance condition is $\omega-k_{\|} v_{\|}-n \omega_{c}=0$, where $k_{\|}$is the longitudinal component of the wave vector and $n$ is an integer (e.g. [Trakhtengerts and Rycroft, 2008]).

To study wave - particle interaction, it is important to know the wave structure along a field line. The main parameter of this structure is the parallel wave number $N$ representing a number of nodes in the wave magnetic field. Nodes of the magnetic field correspond to antinodes of the electric field. Thus, the fundamental harmonic $(N=1)$ has only one magnetic field node located at the equator. Accordingly, the electric field of a wave with $(N=2)$ has an antinode there (Figure 3$)$. The second harmonic $(N=3)$ features a magnetic field antinode and an electric field node at the equator. ULF waves exchange energy with particles mainly through a transverse electric field $E_{\perp}$. Therefore, particles drifting near the equator (equatorial pitch angle $\alpha_{0} \approx 90^{\circ}$ ) can be in resonance with the fundamental harmonic, but not with the second one. Such particles can neither gain energy from a second harmonic wave nor generate it through any instability $\mathrm{A}$ resonance with the fundamental harmonic is the drift resonance (bounce harmonic $K=0$ ).

Following [Southwood and Kivelson, 1982], the drift-bounce resonance condition for various harmonics of the standing wave can be demonstrated in a graphic way. In a reference frame moving with the wave at an angular velocity $\omega / m$ relative to Earth, the wave electric field is stationary. If resonance condition (20) is satisfied, a particle moves from the region of the strong accelerating field of one half-wave to the region of the strong accelerating field of the other half-wave. Concurrently, it either moves through regions of a decelerating field where its amplitude is weak or completely bypass them. For example, in the case of the 


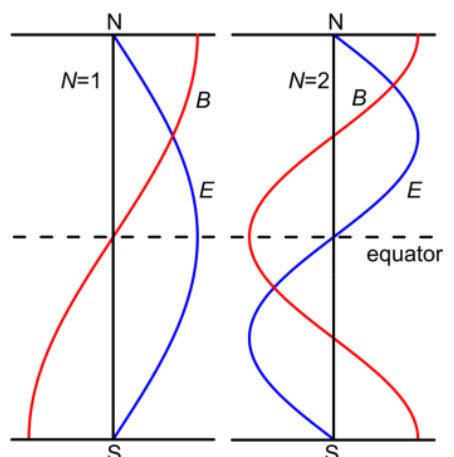

Figure 3. Magnetic and electric fields of the two main harmonics of the Alfvén wave

fundamental harmonic of a standing wave $N=1$ and the second bounce harmonic $K=2$ (Figure 4,a), a particle first crosses the equator in the area of the first half-

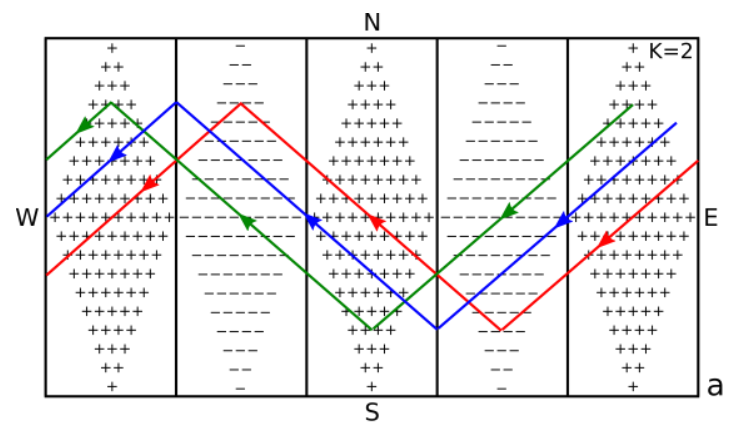

N

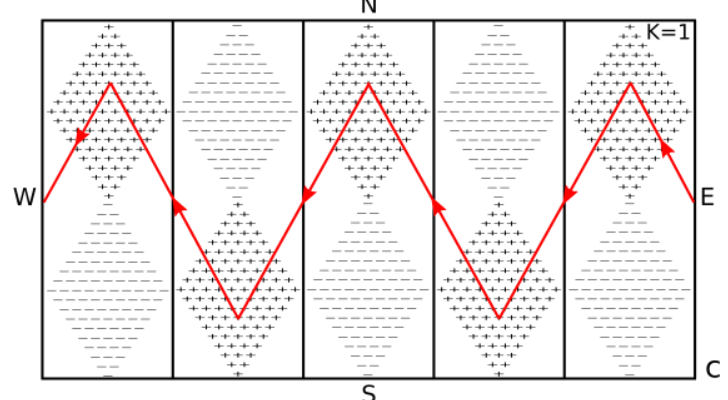

wave. It is accelerated efficiently there. Then it moves through the near-ionospheric region of the next halfwave, where the electric field is decelerating, but weak. Thus, as a particle passes these two half-waves, its resulting acceleration is positive. Obviously, in passing the next pair of half-waves, a particle also accelerates, and so on. The case of $N=1, K=2$ differs from the previous one only in the proton's direction of motion in the wave rest frame (Figure $4, b$ ). In the case of the second harmonic and the first bounce harmonic, a particle moves through half-waves with a strong accelerating field and practically avoids the decelerating field regions (Figure 4,c). If the parameters $N=2$ and $K=2$, a particle experiences acceleration while passing two half-waves successively before being decelerated in the electric field of an opposite sign while passing another two successive half-waves. As a result, its averaged energy is constant (Figure 4, d). Thus, although the resonance
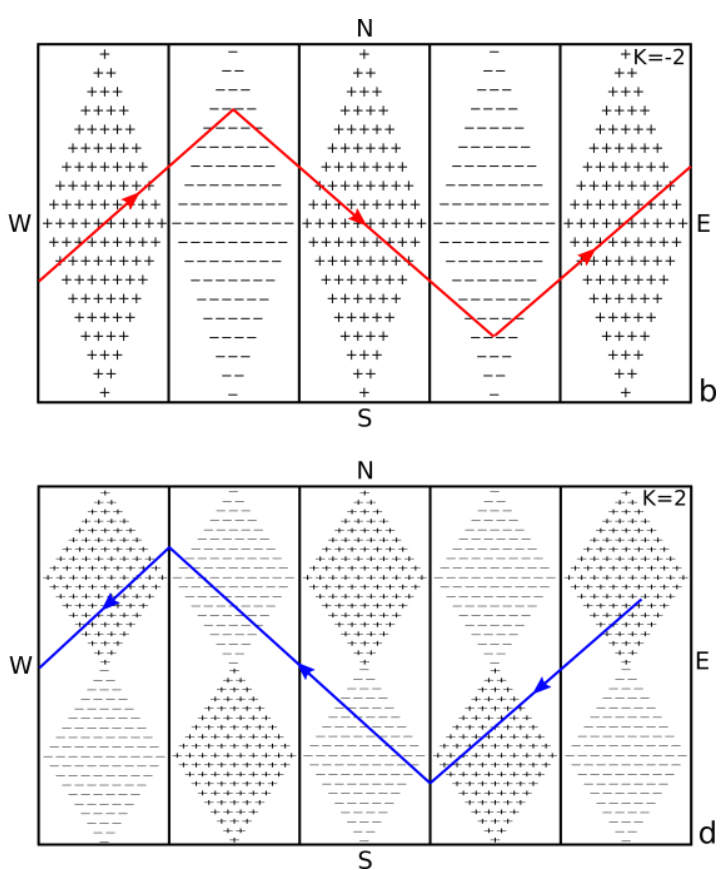

Figure 4. Drift bounce resonance for symmetric and antisymmetric modes with $\mathrm{K}=1,2$ (in the wave rest frame). Pluses represent a westward wave electric field, and minuses indicate an eastward field. The number of pluses or minuses in a horizontal row denotes a field magnitude. The red and green lines show accelerating and decelerating protons' trajectories respectively. The blue line is a trajectory of a proton with a constant averaged energy

condition is satisfied, there is generally no interaction between a particle and a wave. The drift resonance occurs when a particle does not leave a region of strong accelerating field of the same half-wave (Figure 5).

Since the drift and bounce frequencies are proportional to $\varepsilon$ and $\sqrt{\varepsilon}$ respectively, relation (20) can be interpreted as a quadratic equation with respect to the square root of the particle energy. Its solution is the value of the resonant energy of a particle $\varepsilon_{\text {res }}$, i.e. the energy at which a particle can be in the drift-bounce resonance with a wave at given $\omega, m, K$, and $\alpha_{0}$. The dependences of the resonance energy on the wave frequency $\omega$ and the azimuthal wave number $m$ are shown in Figures 6 and 7. Equation (20) has two solutions

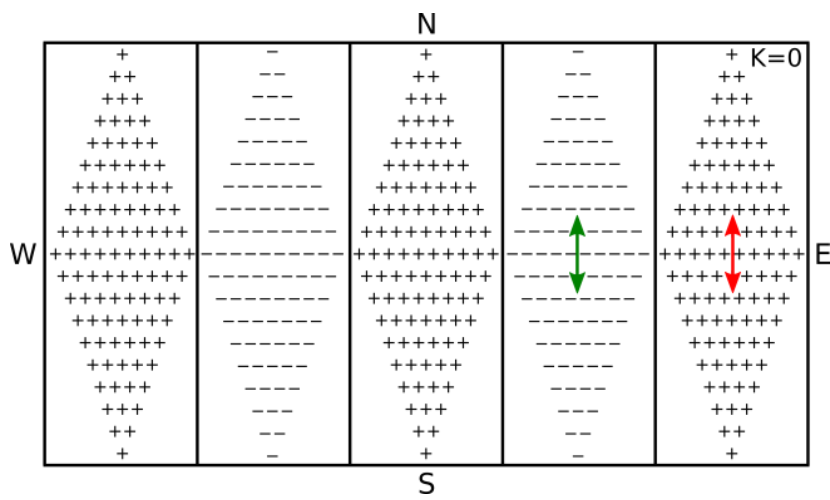

Figure 5. Drift resonance (in the wave rest frame). Designations are the same as in Figure 4 

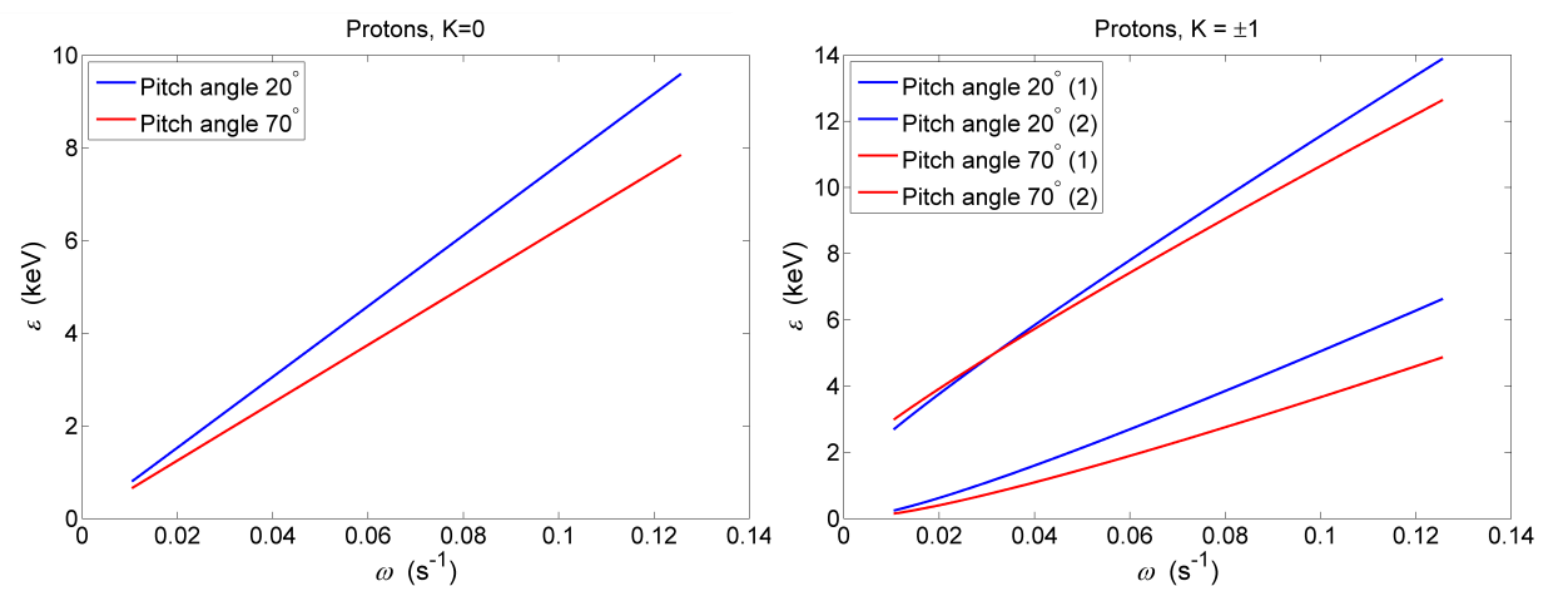

Figure 6. Dependence of the proton resonance energy on the wave frequency for two pitch angle values. Drift resonance (left); drift-bounce-resonance ( $K= \pm 1$ ) (right). Magnetic shell $L=6.6 R_{\mathrm{E}}$, azimuthal wave number $m=-60$
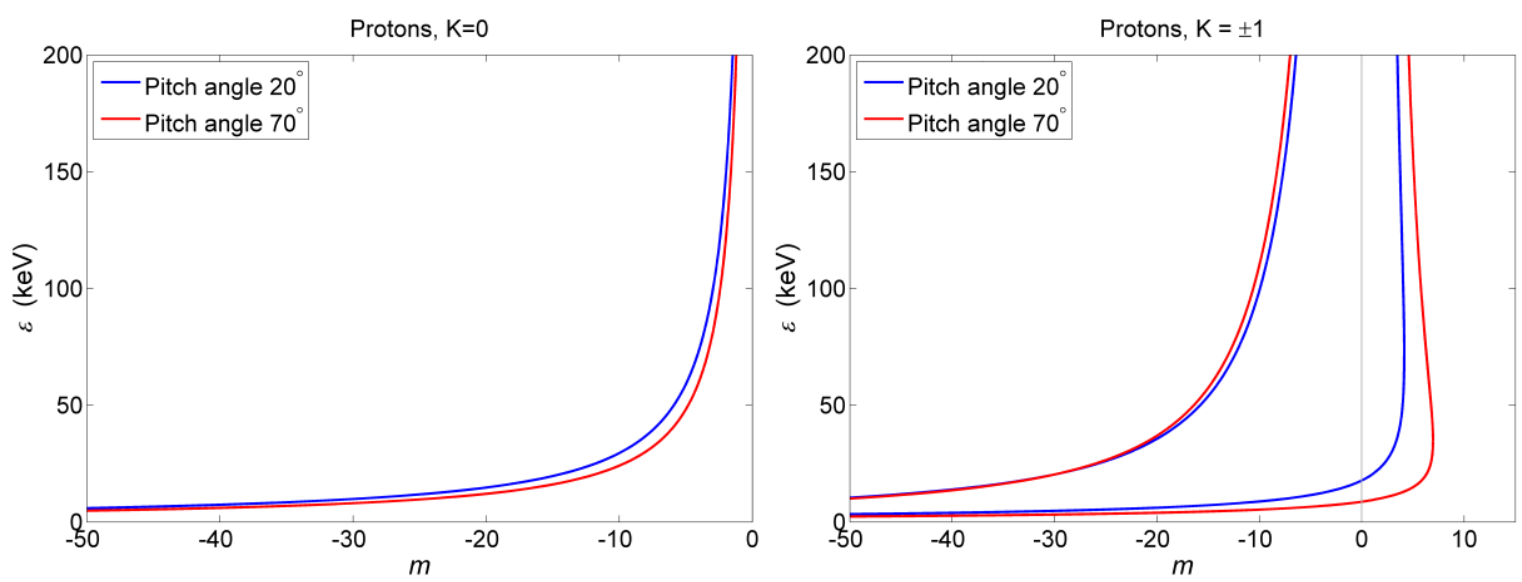

Figure 7. Dependence of the proton resonance energy on the azimuthal wave number for two pitch angle values. Drift resonance (left); drift-bounce resonance $(K= \pm 1)$ (right). Magnetic shell $L=6.6 R_{\mathrm{E}}$, wave period $100 \mathrm{~s}$ (Pc4 range)

in the case of drift-bounce resonance. Note that for electrons only drift resonance is possible: their mass is small, and for Pc4-5 range frequencies (20) has a solution only for $K=0$.

\subsection{Particle transport across magnetic shells}

The interaction with a wave results not only in an increase or decrease in the particle energy, but also in the motion of a particle across magnetic shells. Linearizing expression for the guiding center velocity (3) reveals a radial component of the particle drift velocity. It is determined by the wave electric $\vec{E}$ and magnetic $\vec{B}$ fields:

$$
\delta u_{\mathrm{dr}}=c \frac{E_{\mathrm{a}}}{B_{0}}+v_{\|} \frac{B_{\mathrm{r}}}{B_{0}}-\frac{c \mu}{q B_{0}} i k_{\mathrm{a}} B_{\|},
$$

where $k_{\mathrm{a}}$ is the azimuthal component of the wave vector $\left(k_{\mathrm{a}}=m / L\right.$ at the equator) [Southwood, 1973,1976]. Here, the first term is the electric drift of a particle $\delta u_{E}$ caused by the azimuthal component $E_{\mathrm{a}}$ of the electric field of a wave. Electric drift can be interpreted as a movement of a field line [Longmire, 1963]. With the radial displacement of the field line denoted as $\xi_{\mathrm{r}}$, the electric drift of a particle is

$$
\delta u_{E}=\frac{\partial \xi_{r}}{\partial t}=-i \omega \xi
$$

The second term in (22) is related to a change in the slant of a field line in the meridional direction caused by the radial component of the wave magnetic field $B_{r}$ [Dungey, 1964] (Figure 8). The third term is a particle gradient drift velocity fluctuation associated with variations in the magnetic field absolute value in a wave.

Straightforward yet tedious calculations transform (22) into a relation for the rate of change of the particle's McIlwain parameter:

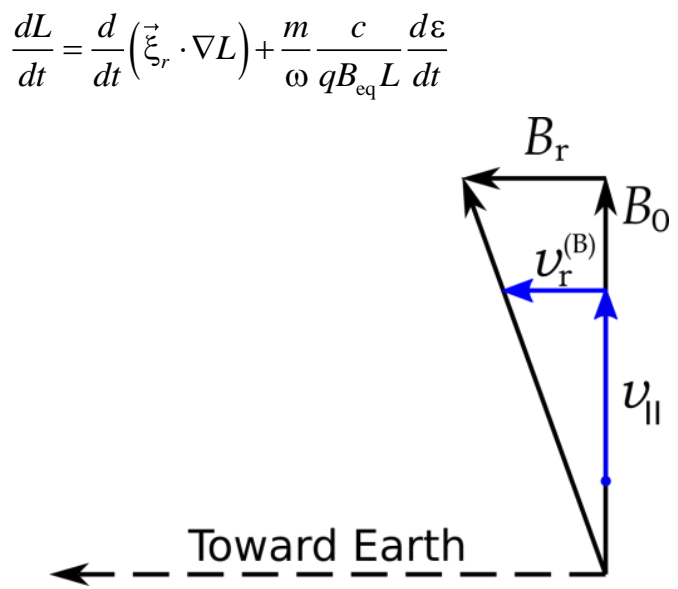

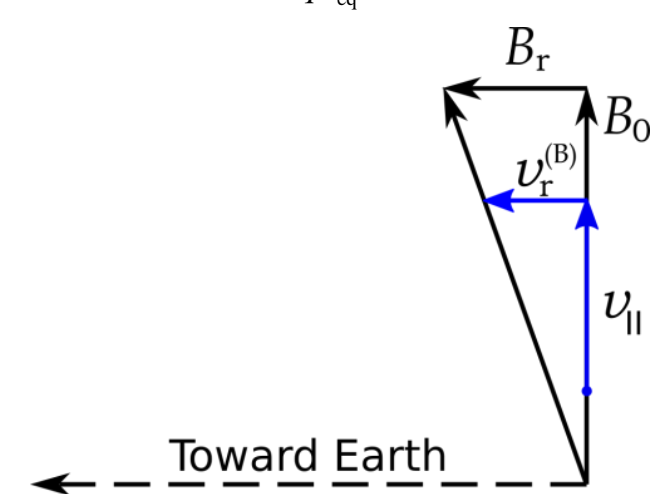

Figure 8. Radial motion of a particle due to a change in the slant of magnetic field lines 
[Southwood, 1973, 1976]. Here, $B_{\text {eq }}$ is the value of the magnetic field at the equator at a given magnetic shell.

Due to the conservation of the first and second adiabatic invariants $\mu, J$, the energy of a particle moving toward Earth increases. A shift in the magnetic shell number by $\delta L$ results in an energy change

$$
\delta \varepsilon=\frac{q B_{\mathrm{eq}} L}{c} \bar{\omega}_{\mathrm{d}} \delta L
$$

[Southwood, 1972]. For ions $\bar{\omega}_{\mathrm{d}}<0$, and for electrons $\bar{\omega}_{\mathrm{d}}>0, q<0$. Thus, the earthward movement of a particle $(\delta L<0)$ causes an increase in its energy regardless of its charge's sign.

\subsection{Dynamics of a near-equatorial particle in the field of an Alfvén wave}

An important case of wave-particle interaction is the motion of a near-equatorial particle (pitch angle, the second adiabatic invariant in the electromagnetic field of an Alfvén wave). In this case, the longitudinal velocity of a particle is insignificant $\left(v_{\|} \approx 0\right)$, and all the particle energy is accumulated in a gyration around a field line: $\varepsilon \approx \varepsilon_{\perp}=M v_{\perp}^{2} / 2$.

The system of equations for near-equatorial particle motion in polar coordinates is

$$
\begin{aligned}
& \frac{d r}{d t}=\frac{E_{\mathrm{a}}}{B_{0}}-\frac{c \mu}{q r B} \frac{\partial B}{\partial \varphi}, \\
& \frac{d \varphi}{d t}=-\frac{E_{\mathrm{r}}}{B_{0}}+\frac{c \mu}{q r B} \frac{\partial B}{\partial r} .
\end{aligned}
$$

Since in the Alfvén wave parallel components of the electric and magnetic fields are small ( $E_{\|} \simeq 0, B_{\|} \simeq 0$ ), the main contribution to the change in the particle energy is made by the transverse electric field:

$$
\frac{d \varepsilon}{d t}=q u_{\mathrm{d}} E_{\mathrm{a}} .
$$

This formula can be given an alternative interpretation. Due to the presence of the azimuthal component of the electric field $E_{\mathrm{a}}$, an electric drift in the radial direction occurs with the velocity $v_{r}=c E_{\mathrm{a}} / B_{0}$. During a time period $\delta t$, a particle is transported over a distance $\delta r=c\left(E_{\mathrm{a}} / B_{0}\right) \delta t$ across magnetic shells. At a new particle location, the magnetic field differs by $\delta B=B_{0}^{\prime} \delta r$ (here the prime means the derivative across a magnetic shell). During this drift, the first adiabatic invariant $\mu=\varepsilon_{\perp} / B_{0}$ is preserved. Then from the $\mu=$ const condition it follows that the energy of the transverse motion changed by $\delta \varepsilon_{\perp}=\mu \delta B$. Substituting $\delta B$ and applying relation for the drift in an inhomogeneous magnetic field (11) at $v_{\|} \approx 0$, can yield (28).

Following [Southwood and Kivelson, 1981], we discuss an approximate solution of (28). Let the amplitude of the electric field be defined by (19). The particle is located at a point with the azimuthal angle $\varphi$. The drift frequency $\omega_{\mathrm{d}}$ of a particle oscillating near the equator is constant; hence $\varphi=\omega_{\mathrm{d}} t+\varphi_{0}$, where $\varphi_{0}$ is the initial azimuthal angle of a particle. Then relation (28) becomes

$$
\frac{d \varepsilon}{d t}=q E_{0} r \omega_{\mathrm{d}} e^{-i\left(\omega-m \omega_{\mathrm{d}}\right) t+i m \varphi_{0}},
$$

where $E_{0}$ is the amplitude of a wave. The solution to this equation is

$$
\Delta \varepsilon=q E_{0} r \omega_{\mathrm{d}} \frac{i}{\omega-m \omega_{\mathrm{d}}}\left[e^{-i\left(\omega-m \omega_{\mathrm{d}}\right) t}-1\right] e^{i m \varphi_{0}} .
$$

As we can see, if $\omega$ and $m \omega_{\mathrm{d}}$ do not coincide, the energy fluctuates around $\varepsilon_{0}$. Otherwise, a secular increase or decrease in energy occurs:

$$
\triangle \varepsilon=q E_{0} r \omega_{\mathrm{d}} e^{i m \varphi_{0}} t
$$

Thus, a continuous transfer of energy from a wave to an equatorial particle, or vice versa, can take place only if drift resonance condition (21) is satisfied.

However, as a result of gaining energy, the particle drift velocity increases, therefore a particle moves faster than a wave. It relocates from a region of the accelerating electric field to a region of the decelerating one. The particle loses energy there, which results in a drift velocity decrease, passing back into the region of the accelerating field. Thus, the wave energy fluctuates even in the drift resonance with particles. Since, as it is shown above, any change in energy is associated with particle motion across magnetic shells, a particle also oscillates in the radial direction. Yet, the resulting acceleration can be non-zero since in reality a wave has a limited duration and a finite spatial extent (as a wave packet). This may be due to both dissipation and the finite time of generation by a wave source and its spatial localization. The dynamics of a near-equatorial particle has been considered in [Degeling et al., 2007, 2008; Gubar', 2010].

\section{THE ROLE OF ULF WAVES IN THE ACCELERATION OF RADIATION BELT PARTICLES}

The wave-particles interaction theory plays an especially important role in the study of radiation belt dynamics. As is known, the outer radiation belt consists of relativistic electrons with energy up to several $\mathrm{MeV}$ ( $1 \mathrm{MeV}$ energy electron has a velocity of $0.86 c$ ). These electrons are often called killer electrons due to their ability to damage orbiting spacecraft [Baker et al., 1987; Baker, 2001; Romanova et al., 2005]. The appearance of such electrons is one of the magnetospheric storms manifestations. Nonetheless, the betatron acceleration associated with a sharp storm time magnetic field change is not a fundamental factor for strengthening the killer electron flows [Pilipenko et al., 2017]. At the turn of the XX and XXI centuries, the first experimental data appeared which indicated that relativistic electron huge energies can be due to ULF waves. Rostoker et al. [1998] and Baker et al. [1998] showed that an increase in relativistic electron fluxes during high geomagnetic activity intervals was preceded by the appearance of large amplitude ULF waves. The relations between 
increasing electron fluxes and ULF waves were also demonstrated in statistical studies [Mathie and Mann, 2000, 2001; Degtyarev et al., 2009]. Moreover, a new geomagnetic index for estimating the magnetosphere disturbance by ULF waves was proposed [Kozyreva et al., 2007; Romanova and Pilipenko, 2009].

Even at relatively low energies, electron velocities are very high due to their low mass. For this reason, the electron bounce frequency is much higher than their drift frequency. This means that drift-bounce resonance condition (20) can only be satisfied for a zero bounce harmonic $(K=0)$. Thus, ULF waves can interact with electrons only in the drift resonance. For the energies characteristic of the outer radiation belt electrons (from several hundred kiloelectronvolt to several megaelectronvolt), drift resonance condition (21) can hold only for relatively low azimuthal wave numbers: $m<10$ for the Pc5 frequency range. This means that the waves responsible for the killer electron acceleration should have an external origin associated with the solar wind, like the solar wind dynamic pressure impulses [Potapov, 2013; Foster et al., 2015] and the KelvinHelmholtz instability [Mathie and Mann, 2001]. Such waves can also penetrate directly from the solar wind [Potapov et al., 2012, 2013].

Let us consider the main mechanisms of radiation belt electron acceleration by ULF waves proposed to date.

\subsection{Acceleration in drift resonance}

The simplest method of the electron acceleration by ULF waves can be summarized in the following way: a wave is excited by a source, an electron gets into a drift resonance with the wave due to interaction with its azimuthal electric field; the electron energy linearly increases with time according to (31). A number of articles have experimentally demonstrated this mechanism [Zong et al., 2009, 2012; Mann et al., 2013; Claudepierre et al., 2013; Foster et al., 2015; Hao et al., 2014, 2019]. In this case, the wave source is usually associated with a solar wind pressure impulse. The impulse initially generates a fast magnetosonic wave, which propagates through the magnetosphere and generates an Alfvén wave on the resonant shell. Numerical modelling of the dynamics of electrons was carried out in [Degeling et al., 2007, 2008].

Another mechanism, which also requires fulfillment of the drift resonance condition for its implementation, has also been proposed in a number of works. In this case, the ULF wave background with random amplitudes and phases in the magnetosphere is assumed to be in the magnetosphere. Entering into a drift resonance with this background, a particle experiences a random walk across magnetic shells, either losing or gaining energy at short temporal intervals. In the end, there is particle diffusion from areas of increased concentration to areas of decreased concentration. As follows from Eq. (25), if the diffusion is earthward, the particle energy increases due to the conservation of the adiabatic invariants $\mu, J$ [Falthammar, 1965, 1968;
Lejosne and Kollmann, 2020]. The diffusion equation has the form [Schulz and Lanzerotti, 1974]:

$$
\frac{\partial f}{\partial t}=L^{2} \frac{\partial}{\partial L}\left[\frac{D_{L L}}{L^{2}} \frac{\partial f}{\partial L}\right] .
$$

Here $f$ is the density of particles in the phase space, $D_{L L}$ is the diffusion coefficient. Following [Brizard and Chan, 2001; Fei et al., 2006], the diffusion coefficient is expressed as

$$
D_{L L}=D_{L L}^{E}+D_{L L}^{B},
$$

where $D f_{L}$ and $D f_{L}$ are the contributions due to fluctuations in the electric and (modulus) magnetic fields, respectively. Analytical expressions for these values have been derived in [Brizard and Chan, 2001; Fei et al., 2006]:

$$
\begin{aligned}
& D_{L L}^{E}=\frac{1}{8 B_{E}^{2} R_{E}^{2}} L^{6} \sum_{m} P_{m}^{E}\left(m \bar{\omega}_{\mathrm{d}}\right) . \\
& D_{L L}^{B}=\frac{\mu^{2}}{8 q^{2} \gamma^{2} B_{E}^{2} R_{E}^{4}} L^{4} \sum_{m} m^{2} P_{m}^{B}\left(m \bar{\omega}_{\mathrm{d}}\right) .
\end{aligned}
$$

Here $B_{E}$ is the magnetic field magnitude at the Earth equator, $\gamma=\left(1-v^{2} / c^{2}\right)^{-1 / 2}$ is the relativistic factor, $P_{m}^{E}$ and $P_{m}^{B}$ are the spectral densities of ULF waves' electric and magnetic fields. The argument $m \bar{\omega}_{\mathrm{d}}$ indicates that spectral densities should be taken for the frequency corresponding to the drift resonance. The functions $P_{m}^{E}$ and $P_{m}^{B}$ should be determined from experimental data [Ozeke et al., 2012]. Empirical analytical expressions for the diffusion coefficients depending on the $L$-shell and the magnetosphere disturbance level $\left(K_{\mathrm{p}}\right.$ index $)$ are given in [Ozeke et al., 2014]. Note that there are some disagreements about the correct form of the diffusion coefficients in theory [Lejosne, 2019].

The diffusion theory can explain a number of basic features of radiation belt electron distribution [Loto'aniu et al., 2006; Shprits et al., 2008; Mann et al., 2012; Su et al., 2015; Ozeke et al., 2018; Sandhu et al., 2021]. At the same time, some researchers express doubts that fundamental premises of this theory (in particular, the stochastic nature of ULF waves, completely stochastic particle motion) correspond fully to reality [Ukhorskiy et al., 2006; Degeling et al., 2007, 2008; Ukhorskiy and Sitnov, 2008]. Probably, the diffusion theory as a whole correctly describes the averaged dynamics of radiation belts. However, in each specific case numerical simulation of the motion of electrons in the field of ULF waves generated by external and internal processes under these specific conditions is required. It may be necessary to take into account the combined effect of ULF waves and higher frequency plasma oscillations, including VLF waves (especially choirs) [O'Brien et al., 2003; Li et al., 2005; Simms et al., 2021]. ULF waves can contribute to the initial acceleration of particles and their transport to the magnetosphere inner regions. They can be locally accelerated by VLF waves to relativistic energies there 
[Pilipenko et al., 2017]. Theoretical and observational arguments in favor of particle acceleration by VLF waves are presented in [Demekhov et al., 2006; Baker et al., 2019; Allison and Shprits, 2020].

In conclusion of this subsection, we note that the drift resonance condition $\omega=m \bar{\omega}_{\mathrm{d}}$ implies the waveparticle interaction is caused by the Alfvén wave electric field azimuthal component $E_{\mathrm{a}}$. This follows directly from energy balance equation (28). However, as noted by Hudson et al. [1999], the acceleration by the radial component $E_{\mathrm{r}}$ is possible as well. It can occur if the geomagnetic field is not axisymmetric. For example, the magnetosphere is compressed from the dayside by the incoming solar wind flow. As is known, nearequatorial particles move along constant magnetic field isolines. The particle's drift trajectory will then be stretched and shifted in the midnight-noon direction, as shown in Figure 9. Imagine, for example, that the Alfvén wave has $m=2$. Let an electron start in the magnetosphere morning sector. Then, drifting in the equatorial plane along the constant geomagnetic field isoline, the electron will move away from Earth, constantly experiencing the accelerating effect of the wave's radial electric field. After passing the midnight-noon line, the electron will begin to approach Earth, but the wave's electric field will also change the sign. This means that the electron will still be accelerated by the radial electric field. Drift resonance condition in such a compressed magnetic field (21) should be replaced by condition

$$
\omega-(m \pm 1) \bar{\omega}_{\mathrm{d}}=0
$$

The electron energy increment calculation for this case was given in [Elkington et al., 2003].

\subsection{Non-resonant acceleration mechanisms}

Several papers have suggested that mechanisms associated with ULF waves, but not requiring waveparticle resonance, may be responsible for the acceleration and radial transfer of radiation belt particles.

To understand the conditions which make it possible, let us find in general the energy acquired by a particle during a gyroperiod $\tau_{\mathrm{c}}$,

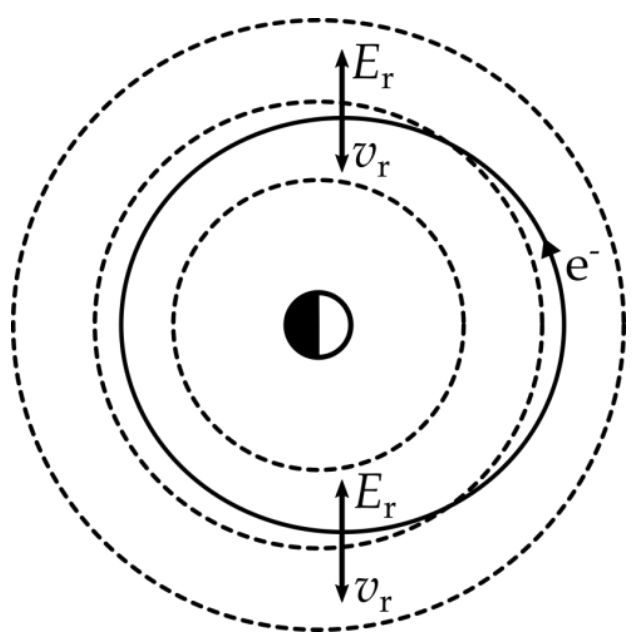

Figure 9. Electron motion in a compressed dipole field. Azimuthal wave number $m=2$ (according to [Hudson et al., 1999; Elkington et al., 2003])

$$
\Delta \varepsilon=q \int_{0}^{\tau_{\mathrm{c}}} E_{\mathrm{a}} u_{\mathrm{d}} d t=q r \int_{0}^{\tau_{\mathrm{c}}} E_{0} e^{-i\left(\omega-m \omega_{\mathrm{d}}\right) t} \omega_{\mathrm{d}} d t
$$

(we limited ourselves to the case of a near-equatorial particle). If the drift resonance condition is not met, at constant values of the wave amplitude $E_{0}$ and the drift frequency $\omega_{\mathrm{d}}$, this integral is zero. The only way to obtain $\Delta \varepsilon \neq 0$ is to assume that the amplitude and/or the drift velocity depend on time.

The particle acceleration by the damping ULF wave electric field is empirically demonstrated in [Zong et al., 2011; Wang et al., 2015]. The damping might have been due to the interaction with the ionosphere or due to the wave energy transfer to particles. The energy increase is maximum when the drift resonance condition holds, but the acceleration occurs even out of the resonance. Indeed, if the initial position of a particle is such that during the first half-period it acquires energy from a wave, in general it accelerates: although the particle loses energy during the next half-period since the field value decreases due to the wave damping, this does not compensate the energy increase during the first halfperiod. The particle energy change for this case was calculated in [Zhou et al., 2016].

An alternative case was examined by Liu et al. [1999]: in integral (37), the particle drift velocitychanges. It is assumed to be associated with the presence of a background of high-frequency plasma waves in the magnetosphere. Interaction with such a background practically does not change the energy of particles, but changes their pitch angles [Tsurutani and Lakhina, 1997]. Since the drift velocity depends on the pitch angle, the interaction with a high-frequency background causes the drift velocity to change and integral (37) to become not equal to zero. Of course, in each specific case, a particle in this process can both gain and lose energy. However, in general, there is a transfer of energy from a wave to particles. It follows from the second principle of thermodynamics: the transfer of energy from a wave to particles corresponds to increased disorder in the wave-particle system. Liu et al. [1999] called this mechanism the magnetic pumping.

Using Equation (37) assumes that the wave is coherent. In this case, the wave is described by a certain frequency and azimuthal wave number. Ukhorskiy et al. [2009] removed this restriction. It was supposed that the wave is divided into small azimuthal sectors with random phase variation from sector to sector. Thus, the wave is azimuthally small-scale, although it does not have a well-defined azimuthal wave number. Similarly, the wave is divided into sectors with random phases by $L$-shells. When an electron enters the wave localization region, it experiences random "shocks" from the wave electric field in different regions. As a result, it makes random walk through the magnetosphere with simultaneous energy change. As shown in that paper, on average, the energy of the particles grows. Thus, Ukhorskiy et al. [2009] justify the possibility of the acceleration of radiation belt particles by azimuthally small-scale waves out of the drift resonance. Numerical simulation of the electron dynamics in the field of poloidal Alfvén waves with $m>>1$ has been carried out in [Degeling et al., 2019; Rankin et al., 2020]. 


\section{STRUCTURE OF ULF WAVES IN MAGNETOHYDRODYNAMICS}

A detailed discussion of the spatial structure of ULF waves in the magnetosphere is beyond the main objectives of this review. However, a brief discussion of this question is crucial for providing an insight into both the wave generation by charged particles and the acceleration of particles by waves or charged particle flux modulation. The structure of waves is primarily studied in the framework of magnetohydrodynamics (MHD) [Alperovich and Fedorov, 2007; Leonovich and Mazur, 2016]. This approach usually involves axially symmetric models of the magnetosphere, which take into account the curvature of field lines, the inhomogeneity of the magnetic field and plasma across magnetic shells and along field lines.

\subsection{Equilibrium and coordinate system}

To study ULF waves in a dipole-like magnetosphere, an axially symmetric coordinate system $\left\{x^{1}, x^{2}, x^{3}\right\}$ is used. It is oriented so that the radial coordinate $x^{1}$ defines a magnetic shell, the azimuthal coordinate $x^{2}$ defines a field line at the shell, and the longitudinal coordinate $x^{3}$ defines a point on a field line (Figure 10).

The distance from Earth's center to the point of intersection of the geomagnetic equator and a field line $L$ (the McIlwain parameter) is used as a radial coordinate; and the azimuthal angle $f$, as an azimuthal coordinate. The physical length of a vector in this coordinate system is $d l_{i}=\sqrt{g_{i}} d x^{i}$. Here, $g_{i}$ is a metric tensor component. The determinant of the metric tensor is $g=g_{1} g_{2} g_{3}$

If the plasma pressure is isotropic, the plasma equilibrium conditions are defined by relations

$$
\begin{aligned}
& \nabla P_{0}=\frac{1}{c} \vec{j}_{0} \times \vec{B}_{0}, \\
& \frac{\beta}{2} \kappa+\kappa_{B}+\frac{1}{R}=0 .
\end{aligned}
$$

Here, $P_{0}, j_{0}$, and $B_{0}$ are the equilibrium values of plasma pressure, current, and magnetic field;

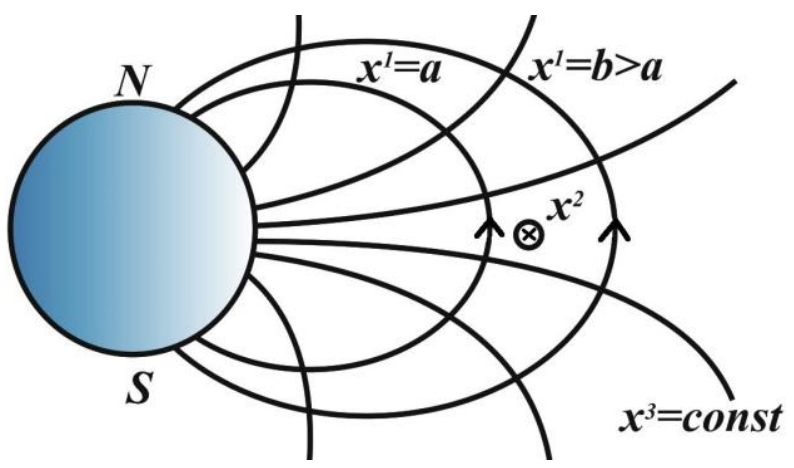

Figure 10. The curvilinear coordinate system oriented by the magnetic field $\beta=4 \pi P_{0} / B_{0}^{2}$ is the plasma pressure to magnetic pressure ratio; $R$ is the local radius of field line curvature; parameters

$$
\begin{aligned}
& \kappa=\frac{1}{\sqrt{g_{1}} P_{0}} \frac{d P_{0}}{d x^{1}}=\frac{1}{\sqrt{g_{1}} P_{0}} \frac{d P_{0}}{d L}, \\
& \kappa_{B}=\frac{1}{\sqrt{g_{1}} B_{0}} \frac{d B_{0}}{d x^{1}}=\frac{1}{\sqrt{g_{1}} B_{0}} \frac{d B_{0}}{d L}
\end{aligned}
$$

characterize the inverse scale of the plasma pressure and magnetic field inhomogeneity.

Relation (38) implies the invariability of the equilibrium plasma pressure $P_{0}$ along a field line. The $B_{0} \sqrt{g_{1} g_{2}}$ parameter defines the magnetic flux per unit area $\left(\delta x^{1}=1, \delta x^{2}=1\right)$ that is perpendicular to a field line.

The relation $\nabla \cdot \vec{B}_{0}=0$ means that this parameter is invariable along a field line. If the McIlwain parameter $L$ and the azimuthal angle $\varphi$ are the coordinates $x^{1}, x^{2}$, it is easy to show that $B_{0} \sqrt{g_{1} g_{2}}=B_{\text {eq }} L$, where $B_{\text {eq }}$ is the magnitude of the magnetic field at the equator of a given magnetic shell.

\subsection{The main equations}

Within the framework of one-fluid magnetohydrodynamics, hot plasma is known to conduct three modes of oscillations: the Alfvén mode, slow and fast magnetic sound. The Alfvén wave dispersion relation is

$$
\omega^{2}=k_{\|}^{2} v_{\mathrm{A}}^{2} \text {. }
$$

Here, $\omega$ is the wave frequency, $k_{\|}$is the component of the wave vector along the magnetic field, $v_{\mathrm{A}}=B_{0} / \sqrt{4 \pi \rho_{0}}$ is the Alfvén velocity, where $B_{0}$ and $\rho_{0}$ are the equilibrium values of the magnetic field and the plasma density. The absence of the transverse dispersion (i.e., the frequency dependence on the transverse component of the wave vector) indicates that the Alfvén mode transfers energy only along the magnetic field. Two types of magnetoacoustic waves are described by dispersion relation

$$
\begin{aligned}
& \omega_{ \pm}^{2}=\frac{1}{2}\left\{k^{2}\left(v_{\mathrm{S}}^{2}+v_{\mathrm{A}}^{2}\right) \pm\right. \\
& \left. \pm \sqrt{k^{4}\left(v_{\mathrm{S}}^{2}+v_{\mathrm{A}}^{2}\right)^{2}-4 k_{\|}^{2} k^{2} v_{\mathrm{S}}^{2} v_{\mathrm{A}}^{2}}\right\},
\end{aligned}
$$

where $v_{\mathrm{s}}=\sqrt{\gamma P_{0} / \rho_{0}}$ is the speed of sound, $\gamma$ is the adiabatic exponent. The upper and lower signs refer to fast and slow magnetoacoustic waves (FMS and SMS) respectively. If $k_{\perp} \gg k_{\|}$the dispersion relations for these modes transform into

$$
\begin{aligned}
& \omega^{2}=k^{2}\left(v_{\mathrm{A}}^{2}+v_{\mathrm{S}}^{2}\right), \\
& \omega^{2}=k_{\|}^{2} v_{\mathrm{C}}^{2}
\end{aligned}
$$

respectively. Here 


$$
v_{\mathrm{C}}=\frac{v_{\mathrm{A}} v_{\mathrm{S}}}{\sqrt{v_{\mathrm{S}}^{2}+v_{\mathrm{A}}^{2}}}
$$

is the speed of SMS. As one can see, SMS, like the Alfvén mode, propagates predominantly along field lines.

In inhomogeneous plasma, all three MHD modes are coupled; therefore, they are described by a system of interdependent wave equations.

In case of the azimuthally small-scale limit of the Pc4-5 range, the FMS propagation region is narrowly localized near the magnetopause. Thus, the role of FMS may be neglected when studying waves inside the magnetosphere [Leonovich and Mazur, 2016]. Besides, the characteristic frequencies of SMS are significantly lower than those of Alfvén waves [Cheremnykh and Parnowski, 2004]. Hence, coupling with SMS has a relatively small effect on propagation of Alfvén waves. The transverse component of the electric field of Alfvén waves is not vortex, that is, it can be represented in the form of a transverse gradient of a scalar function:

$$
\vec{E}=-\nabla_{\perp} \Phi,
$$

[Tamao, 1984b; Klimushkin, 1994], where the sign $\perp$ marks the direction perpendicular to a field line. Based on the MHD equations, the following relation for propagation of Alfvén waves can be derived:

$$
\partial_{1} \hat{L}_{\mathrm{T}}(\omega) \partial_{1} \Phi-m^{2} \hat{L}_{\mathrm{P}}(\omega) \Phi=q
$$

[Klimushkin et al., 2004] Here,

$$
\begin{aligned}
& \hat{L}_{\mathrm{T}}(\omega)=\partial_{3} \frac{g_{2}}{\sqrt{g}} \partial_{3}+\frac{\sqrt{g}}{g_{1}} \frac{\omega^{2}}{v_{\mathrm{A}}^{2}}, \\
& \hat{L}_{\mathrm{P}}(\omega)=\partial_{3} \frac{g_{1}}{\sqrt{g}} \partial_{3}+\frac{\sqrt{g}}{g_{2}}\left(\frac{\omega^{2}}{A^{2}}+\eta\right)
\end{aligned}
$$

are the toroidal and poloidal mode operators respectively, $\eta$ is a correction related to the plasma pressure

$$
\eta=-\frac{2}{R}\left(\frac{J}{B}+\frac{2}{R} \frac{v_{\mathrm{S}}^{2}}{v_{\mathrm{A}}^{2}}\right),
$$

$q$ is a wave source. An example of a source can be external currents in the magnetosphere $j_{\text {ext }}$ [Zolotukhina, 1974; Pilipenko et al., 2001].

In this case, the right-hand side of (48) is

$$
q=\frac{4 \pi i \omega}{c^{2}} \nabla_{\perp} \cdot \vec{j}_{\mathrm{ext}},
$$

[Mager and Klimushkin, 2007, 2008]. Fluctuating fieldaligned currents in the ionosphere and magnetosphere can also act as wave sources [Leonovich and Mazur, 1996; Pilipenko et al., 2016].

Equation (48) should be complemented with the boundary condition for the ionosphere. Due to the high conductivity of ionospheric plasma, in the first approximation the Alfvén wave is reflected from the ionosphere. Therefore, the boundary condition has the form

$$
\Phi\left(x_{ \pm}^{3}\right)=0,
$$

where $x_{ \pm}^{3}$ is the point of intersection of the ionosphere and a field line. A wave that satisfies this boundary condition should be standing along a field line. A more general form of the boundary conditions (in the presence of finite conductivity and the external currents in the ionosphere) is given in [Leonovich and Mazur, 1996].

If the radial scale of the disturbance is much smaller than the azimuthal scale, the structure of the Alfvén wave is determined by the first term of (48). As follows from (47), in this case the radial component dominates in the electric field of the wave. An Alfvén wave with such polarization is called toroidal. The wave structure is approximately expressed as

$$
\Phi \approx R\left(x^{1}\right) T_{N}\left(x^{3}\right),
$$

where the first and second factors describe the structure across magnetic shells and the longitudinal structure respectively. The $N$ index means the number of a harmonic along a field line. The parallel structure of the toroidal mode can be found from equation

$$
L_{\mathrm{T}}(\omega) T_{N}=0
$$

under boundary conditions $T_{N}\left(x_{ \pm}^{3}\right)=0$. The solution to (55) is possible at certain frequencies - the eigenvalues of the equation $\Omega_{\mathrm{TN}}\left(x^{1}\right)$ (toroidal frequency). They depend on the radial coordinate $x^{1}$. For a fixed wave frequency $\omega$ (for a certain $N$ ) the solution is possible only at the magnetic shell $x_{\mathrm{TN}}^{1}$, where the wave frequency is equal to the toroidal frequency $\Omega_{\mathrm{TN}}\left(x^{1}\right)$

$$
\omega=\Omega_{\mathrm{TN}}\left(x^{1}\right) .
$$

The magnetic shell $x_{\mathrm{T} N}^{1}$ is called a toroidal surface. Near this surface, the wave field has a singularity

$$
\Phi \propto \ln \left(x^{1}-x_{\mathrm{T} N}^{1}\right) .
$$

The presence of this singularity was first established within the framework of one-dimensional inhomogeneous magnetosphere models that take into account only plasma inhomogeneity across magnetic shells [Southwood, 1974; Chen and Hasegawa, 1974]. Further work showed that this singularity also existed in two-dimensionally inhomogeneous models with the curvature of field lines and the longitudinal inhomogeneity of plasma and magnetic field taken into account [Lifshits and Fedorov, 1986; Chen and Cowley, 1989]. In a three-dimensionally inhomogeneous magnetosphere model (when the azimuthal inhomogeneity is also taken into account), the form of the singularity can be more complex [Mager and Klimushkin, 2021].

When the azimuthal scale of the disturbance is much smaller than the radial one, the structure of the wave field is determined by the second term of (48). In this case, the azimuthal component dominates in the electric field of the wave. The Alfvén wave of this type is called poloidal. The wave structure is approximately expressed in relation

$$
\Phi \approx R\left(x^{1}\right) P_{N}\left(x^{3}\right),
$$

where the $P_{N}$ function is a solution to 


$$
\hat{L}_{\mathrm{P}}(\omega) P_{N}=0
$$

under the boundary conditions $P_{N}\left(x_{ \pm}^{3}\right)=0$.

Eigenvalues of (59) can be denoted as $\Omega_{\mathrm{PN}}\left(x^{1}\right)$ that is the poloidal frequency. Its dependence on the radial coordinate $x^{1}$ is defined by the parameters that are the functions of this coordinate (Alfvén speed, components of the metric tensor) included in the poloidal operator $\hat{L}_{\mathrm{P}}$. The coordinate of so-called poloidal surface $x^{1}$ can be derived from equation

$$
\omega=\Omega_{\mathrm{PN}}\left(x^{1}\right) \text {. }
$$

In the major part of the magnetosphere, the functions $\Omega_{\mathrm{T} N}\left(x^{1}\right), \quad \Omega_{\mathrm{PN}}\left(x^{1}\right) \quad$ are decreasing. If $\Omega_{\mathrm{T} N}\left(x^{1}\right)>\Omega_{\mathrm{PN}}\left(x^{1}\right)$, the poloidal surface is closer to Earth than the toroidal one: $x_{\mathrm{TN}}^{1}>x_{\mathrm{PN}}^{1}$ (Figure 11), and vice versa. Numerical solution of equations $(55,59)$ shows that in cold plasma (with $\beta=0$ ) the toroidal frequency is always greater than the poloidal one [Cummings et al., 1969; Leonovich and Mazur, 1993]. When the finite pressure is taken into account, both situations are possible $\Omega_{\mathrm{TN}}\left(x^{1}\right)>\Omega_{\mathrm{PN}}\left(x^{1}\right)$, as well as $\Omega_{\mathrm{TN}}\left(x^{1}\right)<\Omega_{\mathrm{PN}}\left(x^{1}\right)$, depending on the pressure value and its gradient.

With not very high pressure $(\beta \ll 1)$, the frequency difference is rather small, the longitudinal structure of the toroidal and poloidal modes is also similar [Klimushkin et al., 2004]. In this case, the radial structure of the wave is approximately described by relation

$$
\begin{aligned}
& \partial_{1}\left[\omega^{2}-\Omega_{\mathrm{TN}}^{2}\left(x^{1}\right)\right] \partial_{1} R_{N}- \\
& -k_{\mathrm{a}}^{2}\left[\omega^{2}-\Omega_{\mathrm{PN}}^{2}\left(x^{1}\right)\right] R_{N}=q_{N} .
\end{aligned}
$$

Here,

$$
\begin{aligned}
& q_{N}=\int_{x_{-}^{3}}^{x_{+}^{3}} q T_{N} d x^{3}, \\
& k_{\mathrm{a}}^{2}=m^{2} \int_{x_{-}^{3}}^{x_{+}^{3}} \frac{\sqrt{g}}{g_{2}} \frac{T_{N}^{2}}{v_{\mathrm{A}}^{2}} d x^{3} .
\end{aligned}
$$

We can see that the parameter $k_{\mathrm{a}}$ has the dimension of a wave vector. The $2 \pi k_{\mathrm{a}}^{-1}$ parameter is approximately equal to the azimuthal wavelength averaged along the field line.

Using the Wentzel-Kramers-Brillouin (WKB) approximation in the radial coordinate, it is easy to obtain the radial component of the wave vector from (61):

$$
k_{1}^{2}=-k_{\mathrm{a}}^{2} \frac{\omega^{2}-\Omega_{\mathrm{PN}}^{2}\left(x^{1}\right)}{\omega^{2}-\Omega_{\mathrm{T} N}^{2}\left(x^{1}\right)} .
$$

The radial component of the wave vector is seen to tend to infinity near the toroidal surface, and it tends to zero near the poloidal one.

As mentioned above, in a homogeneous plasma the magnetic field of the Alfvén wave oscillates in the direction perpendicular to the equilibrium magnetic field $B_{0}$. However, in an inhomogeneous plasma with the

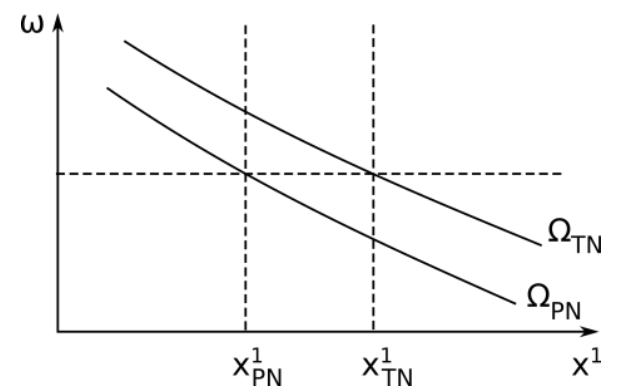

Figure 11. Mutual position of toroidal and poloidal surfaces. A case with $\Omega_{\mathrm{TN}}\left(x^{1}\right)>\Omega_{\mathrm{PN}}\left(x^{1}\right)$ is shown

finite pressure taken into account, the Alfvén wave has a parallel component of the magnetic field $B_{3}$

$$
B_{3}=\frac{c m}{\omega} \frac{g_{3}}{\sqrt{g}} \frac{R_{\eta}}{2} \Phi
$$

[Klimushkin et al., 2004].

\subsection{Structure of Alfvén waves in the region of monotonic variation of toroidal and poloidal frequencies}

The solution of (61) depends on the form of functions $\Omega_{\mathrm{TN}}\left(x^{1}\right), \quad \Omega_{\mathrm{PN}}\left(x^{1}\right)$. They decrease in the major part of the magnetosphere (Figure 11). Leonovich and Mazur [1993] showed that the wave localization region is limited by the region between the poloidal and toroidal surfaces (Figure 12). The wave energy propagates across magnetic shells. A wave is generated in the vicinity of the poloidal surface (for example, by external currents in the magnetosphere or in the ionosphere) and moves toward the toroidal surface. Referring to (63), as a wave approaches the toroidal surface the radial wavelength decreases. In contrast to the homogeneous plasma case, the wave energy propagates across field lines in both the radial and azimuthal directions. Near the poloidal surface, the mode is completely damped due to the finite conductivity of the ionosphere. An oscillation of this type is presumably described in [Leonovich et al., 2015].

When the azimuthal inhomogeneity is taken into account, the range of space enclosed by the poloidal and toroidal surfaces retains the meaning of the region where wave propagation is possible. However, the toroidal surface loses the role of the resonant surface and the wave energy accumulator. Instead, a separatrix surface located between the poloidal and toroidal surfaces appears which takes this role. Wave energy generated by intramagnetospheric processes near the poloidal and toroidal surfaces propagates toward the separatrix surface and dissipates at both its sides [Klimushkin et al., 1995; Mager and Klimushkin, 2021].

So far, we have only considered monochromatic waves, i.e. waves with fixed frequency $\omega$. The opposite case is an impulse-generated wave. If the frequency profiles $\Omega_{\mathrm{TN}}\left(x^{1}\right)$ and $\Omega_{\mathrm{PN}}\left(x^{1}\right)$ are monotonic, a phenomenon called phase mixing occurs [Radoski, 1974]. Let the wave have poloidal polarization at the initial 


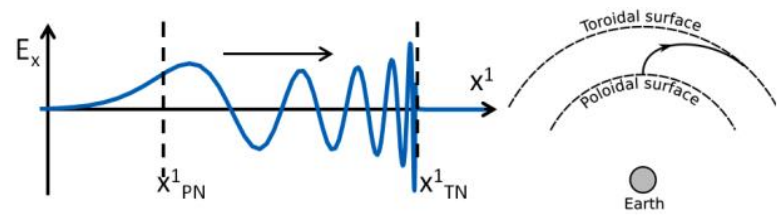

Figure 12. Structure of an azimuthally small-scale Alfvén wave across magnetic shells (left). Path of azimuthally smallscale Alfvén wave propagation from poloidal to toroidal surfaces (right)

moment. Since each magnetic shell oscillates with its own frequency, phases of these oscillations diverge rapidly relative to each other, and the radial wavelength is rapidly decreasing (Figure 13). As a result, a wave becomes toroidally polarized [Mann and Wright, 1995]. The time interval $\tau_{\text {tr }}$ of the wave transformation is estimated by relation

$$
\tau_{\mathrm{tr}} \sim \frac{m}{L}\left(\frac{d \Omega_{\mathrm{TN}}}{d L}\right)^{-1} .
$$

Consideration of the dipole configuration influences this process insignificantly: the transformation of the polarization from poloidal to toroidal is accompanied by an appropriate change in the instantaneous frequency from poloidal $\Omega_{\mathrm{PN}}$ to toroidal $\Omega_{\mathrm{TN}}$ throughout magnetic shells [Leonovich and Mazur, 1998].

Observational features of the Alfvén wave transformation due to phase mixing are presented in [Sarris et al., 2009; Zolotukhina et al., 2008; Wei et al., 2019]. However, such transformations are observed only in exceptional cases: polarization usually remains poloidal. Choi and Lee [2021] suppose that this is due to the fact that poloidal waves occur mainly in regions with a small transverse gradient of the Alfvén speed. On the other hand, it might be explained by the nonmonotonic frequency profile $\Omega_{\mathrm{PN}}(L)$ in the regions with the best conditions for generation of such waves.

The theory of these waves is discussed in the next subsection.

\subsection{Transverse resonator for Alfvén waves}

Until now, we have examined the case with monotonic profiles of $\Omega_{\mathrm{TN}}\left(x^{1}\right), \Omega_{\mathrm{PN}}\left(x^{1}\right)$. In this case, the wave propagation region is limited by the toroidal and poloidal surfaces in the radial coordinate. However, the Alfvén wave can also be localized in the vicinity of extrema of these functions. Expanding the square of the poloidal frequency in a Taylor series near the extremum of $\Omega_{\mathrm{PN}}\left(x^{1}\right)^{2}$ at a point $x_{0}^{1}$ yields the value of the radial component of the wave vector

$$
\begin{aligned}
& k_{1}^{2}=-\frac{k_{\mathrm{a}}^{2}}{\Omega_{\mathrm{PN}_{0}}^{2}-\Omega_{\mathrm{TN}_{0}}^{2}} \times \\
& \times\left\{\left(\omega^{2}-\Omega_{\mathrm{PN}_{0}}^{2}\right)-\frac{1}{2}\left[\Omega_{\mathrm{PN}}\left(x^{1}\right)^{2}\right]^{\prime \prime}\left(x^{1}-x_{0}^{1}\right)^{2}\right\},
\end{aligned}
$$

where the 0 index marks the value of a function near the extremum, and the prime denotes differentiation over $x^{1}$. With the square of the resonator width

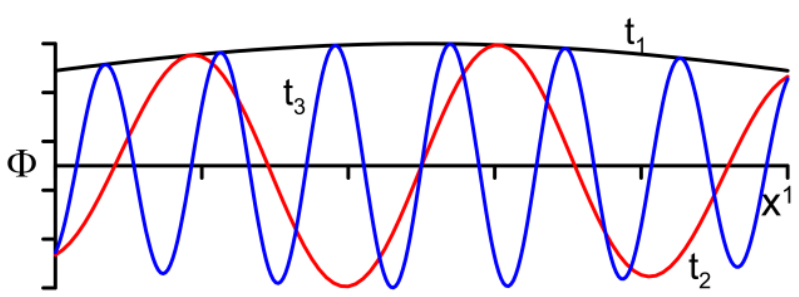

Figure 13. Phase mixing: time variation in the radial structure of a pulse-generated Alfvén wave. A wave structure at three consecutive time points is shown $\left(t_{3}>t_{2}>t_{1}\right)$

$$
a^{2}(\omega)=\frac{2\left(\omega^{2}-\Omega_{P N_{0}}^{2}\right)}{\left[\Omega_{P N}\left(x^{1}\right)^{2}\right]^{\prime \prime}},
$$

and $x=x^{1}-x_{0}^{1}$ relation (66) can be rewritten as:

$$
k_{1}^{2}\left(x^{1}, \omega\right)=-\frac{1}{2} k_{\mathrm{a}}^{2}\left[\Omega_{\mathrm{PN}}\left(x^{1}\right)^{2}\right]^{\prime \prime} \frac{a^{2}(\omega)-x^{2}}{\Omega_{\mathrm{PN}_{0}}^{2}-\Omega_{\mathrm{TN}_{0}}^{2}} .
$$

The wave localization region (where $k_{1}^{2}>0$ ) is seen to be bounded by two poloidal surfaces, i.e. the mode is trapped in a resonator across magnetic shells. The width of the resonator determines the discrete spectrum of the Alfvén wave. It can be found using the Bohr-Sommerfeld quantization rule

$$
\oint k_{1}\left(x^{1}, \omega\right)=2 \pi\left(n+\frac{1}{2}\right) \text {. }
$$

Here, $n$ is the radial harmonic number in the resonator. Figure 14 depicts eigenharmonics of the resonator. The resonator can be located near the maximum of the $\Omega_{\mathrm{PN}}\left(x^{1}\right)^{2}$ function if $\Omega_{\mathrm{PN}_{0}}>\Omega_{\mathrm{TN}_{0}}$, and near the minimum if $\Omega_{\mathrm{PN}_{0}}<\Omega_{\mathrm{TN}_{0}}$ [Vetoulis and Chen, 1994; Leonovich and Mazur, 1995; Klimushkin, 1998; Klimushkin et al., 2004]. The former can take place at the outer edge of the plasmapause with the finite plasma pressure and moderate pressure gradient; the latter can exist at the inner edge of the plasmapause with negligible pressure, or slightly outside the ring current if the pressure is finite and its gradient is significant. The wave energy in the transverse resonator propagates in the azimuthal direction. Giant pulsations (Pgs, poloidal Pc4 waves with moderately high azimuthal wave numbers $m \sim 20$ ) are likely to be eigenharmonics of the resonator at the outer edge of the plasmapause [Mager, Klimushkin, 2013].

If a source of an Alfv'en wave within a transverse resonator is a pulse, all transverse harmonics of the resonator are excited simultaneously, each at its own frequency $\Omega_{n}$. The summation of harmonics with different frequencies and different spatial structures results in harmonic beating. They are accompanied by chaotic changes in the radial structure of the wave [Mager and Klimushkin, 2013]. Such a situation is probably observed in [Yeoman et al., 2012; Mager et al., 2018]. 


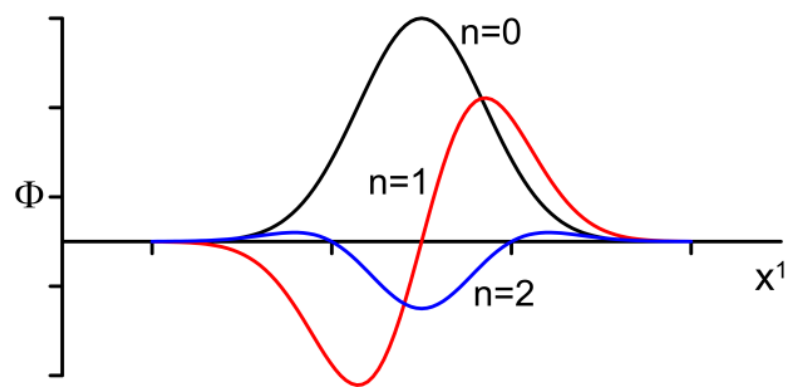

Figure 14. Structure of three main radial harmonics in a transverse Alfvén resonator

\subsection{Alfvén wave generation by a moving source}

We have mentioned above that Alfvén waves can be generated by external currents in plasma. For the magnetosphere, this was first supposed in [Zolotukhina, 1974; Guglielmi and Zolotukhina, 1980]. Mager and Klimushkin [2007] considered a scenario with a current produced by a cloud of charged particles injected during a substorm and drifting in the azimuthal direction at an angular velocity $\omega_{\mathrm{d}}$ (Figure 15). The wave field generated by such a source was calculated in [Mager and Klimushkin, 2008].

It was shown there that at a given point in the magnetosphere, a wave appears at the moment of the particle arrival. At a given magnetic shell, the frequency $\Omega(L)$ of a wave slowly changes from the poloidal to the toroidal eigenfrequency of that $L$-shell. The azimuthal wave number in this case is

$$
m=\frac{\Omega}{\omega_{\mathrm{d}}} .
$$

This relationship implies that the wave phase is constant in the particle frame of reference. Relation (70) coincides with the drift resonance condition, yet it has a completely different physical meaning: the drift resonance condition means that a particle interacts most effectively with the electromagnetic field of a wave with a certain $m$ given that the particle energy satisfies drift resonance condition (21); Equation (70) means that a cloud of particles with a given energy (and, therefore, a given drift frequency) generates a wave whose $m$ satisfies this relation.

Zolotukhina et al. [2008] studied in detail the temporal behavior of the magnetic field of a Pc5 wave that occurred at the geostationary orbit simultaneously with a cloud of substorm-injected particles. They showed that its behavior is consistent with the theory developed by Mager and Klimushkin [2008]. In the statistical study by James et al. [2013], 83 wave events associated with substorms have been examined. It was found that in each case a wave appeared simultaneously with arrival of particles at a measurement point, and relation (70) held for all events. Waves observed to the west (east) of the onset propagated to the west (east), i.e. they moved away from the onset point. This is consistent with wave generation by substorm-injected protons and electrons respectively. Geomagnetic and riometric ground-based observations of azimuthally propagating Pi3

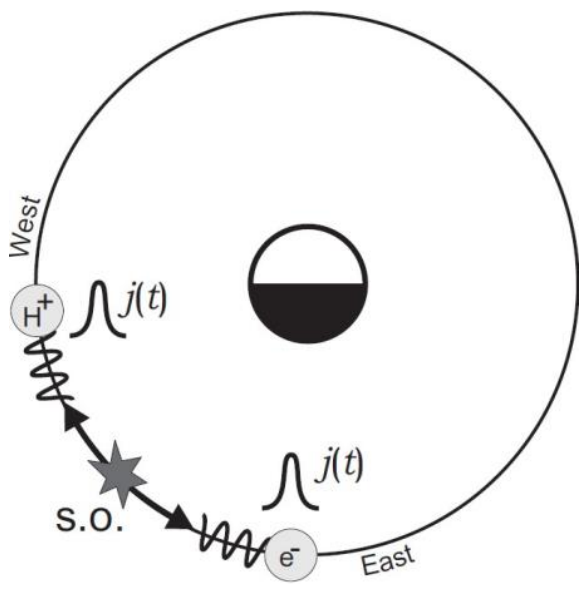

Figure 15. Scheme of wave generation by a moving cloud of injected particles [James et al., 2013]. Substorm onset point is marked by S.O.

pulsations were shown in [Moiseev et al., 2020]. Their velocities corresponded to the propagation velocities of electrons injected during a substorm. Thus, we can conclude that for many events, the theory of a moving source generally corresponds to ULF wave observations.

Additional empirical arguments in favor of Pc5 and $\mathrm{Pi} 2$ wave generation by substorm-injected particles were provided in [Saka et al., 1992, 1996].

\section{ULF WAVES IN KINETICS. ULF WAVE INCREASE DUE TO RESONANT INTERACTION WITH ENERGETIC PARTICLES}

Since magnetospheric plasma is collisionless, the applicability of MHD for its description is questionable. The kinetic approach describing magnetospheric plasma is more adequate, especially at finite plasma pressure. From the point of view of the review, it is of particular importance that the kinetic approach automatically takes into account the resonant wave-particle interaction. Among the important results in kinetics are the existence of oscillation modes that have no MHD analogs [Mikhailovskii and Fridman, 1967; Tajiri, 1967; Xia et al., 2017] and the possibility of wave excitation by instabilities caused by the wave-particle resonance [Korablev and Rudakov, 1968; Hasegawa, 1969; Southwood et al., 1969; Mikhailovskii and Pokhotelov, 1975, 1976].

To study oscillations with frequencies below the ion gyrofrequency, gyrokinetics is usually used. It is a version of kinetics where Vlasov equations are averaged over the gyrophase [Antonsen and Lane, 1980; Catto et al., 1981]. In gyrokinetics, the wave field is described by three variables, one of which $(\Phi)$ describes both the transverse magnetic wave field and the transverse electric wave field; the second, the parallel magnetic field $B_{\|}$; and the third, the parallel electric field $E_{\|}$ [Chen and Hasegawa, 1991]. The variable $\Phi$ is related to the magnetic vector potential $\vec{A}$ by the relation $A_{\|}=-(i c / \omega) \partial \Phi / \partial l_{\|}$. In gyrokinetics, the Coulomb calibration is chosen: $\nabla \cdot \vec{A}=0$. 
The solution of gyrokinetic equations is defined by the equilibrium distribution function $F(\varepsilon, \mu)$. It is usually assumed that the ion plasma component consists of two fractions, cold and hot particles. The cold particles (energy $\varepsilon \sim 100 \mathrm{eV}$ ) determine the plasma density and its inertia. The hot particles (energy $\varepsilon \sim 10-$ $100 \mathrm{keV}$ ) determine the plasma pressure and are responsible for its stability. It is only the hot particles which take part in the resonant interaction with ULF waves. If the plasma pressure is isotropic, the distribution function is independent of the magnetic moment.

It is often assumed that there is an admixture of the cold electrons in plasma (for example, of ionospheric origin). Due to the very small electron mass, the cold electrons are rapidly distributed along a magnetic field line until all points along the field line acquire an equal electrostatic potential. In this case, the wave parallel electric field is negligible, $E_{\|}=0$. We will use this approximation further on. Note that the assumption of the vanishingly small value of the parallel electric field makes slow magnetosonic waves impossible to exist since in kinetics this mode occurs only when the finite $E_{\|}$component is taken into account [Klimushkin and Kostarev, 2012].

\subsection{Instability factors}

Before we proceed to the description of modes of plasma oscillations in kinetics, it is worth focusing on the general plasma instability condition leading to the generation of ULF waves. In this issue, we follow the works [Huba and Drake, 1981, 1982].

It would be sufficient to consider a simplified model in which field lines are straight, but there is a magnetic field gradient $\nabla B_{0}$ directed along the $\mathrm{X}$-axis (Figure 16) [Huba and Drake, 1981]. In this case, the drift velocity of particles is determined by expression

$$
u_{\mathrm{d}}=\frac{v_{\perp}^{2}}{2 \omega_{\mathrm{c}}} \frac{\partial \ln B_{0}}{\partial x}
$$

and directed along the positive direction of the $\mathrm{Y}$-axis. Let us turn to the reference system associated with the wave. Consider two particles located in positions 1 and 2 , differing in the direction of the wave electric field: $E_{y}>0$ and $E_{y}<0$ respectively. Let energies of these particles correspond to the drift resonance: the particles drift with a speed equal to the wave phase velocity along the $\mathrm{Y}$-axis:

$$
u_{\mathrm{d}}=\frac{\omega}{k_{y}} \text {. }
$$

In the wave's reference system, each resonant particle is affected by a stationary electric field, which either slows it down or accelerates it.

Particle 1 experiences electric drift with velocity $u_{E}=c E / B_{0}$ in the positive direction of the X-axis. At the same time, the magnetic field grows at the particlelocation. From the constant magnetic moment $\mu=v_{\perp}^{2} /\left(2 B_{0}\right)$ it

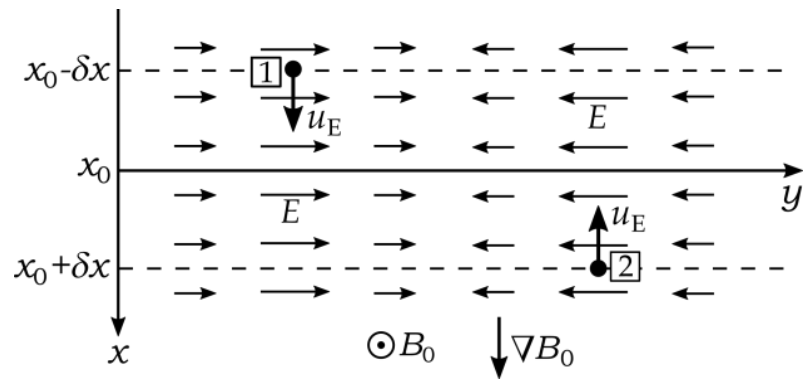

Figure 16. Wave electric field (in the wave frame)

follows that the particle energy (enclosed in rotation around field lines with speed of $v_{\perp}$ ) increases. Consequently, this particle accelerates, gaining energy from the wave. During the time $\delta t$, the particle energy increases by

$$
\delta \varepsilon=q u_{\mathrm{d}} E \delta t=q \frac{\omega}{k_{y}} E \delta t .
$$

Here, it is taken into account that ratio (72) is satisfied in the resonance. In order to be in the position $x_{0}$ at some time, the particle must start from a point with the coordinate $x_{0}-\delta x$, where

$$
\delta x=u_{E} \delta t=c \frac{E}{B_{0}} \delta t .
$$

Particle 2 experiences electric drift with the same velocity $u_{E}=c E / B_{0}$ in the negative direction of the $\mathrm{X}$ axis. Since the magnetic field at the particle location decreases, the particle energy must decrease to save the magnetic moment. Consequently, this particle slows down, transferring its energy to the wave. The decrease in energy over the time period $\delta t$ is also calculated by Equation (73), but the value of $\delta t$ should be taken with the opposite sign. In order to appear in the position $x_{0}$ at some time instant, the particle must start from a point with the coordinate $x_{0}+\delta x$, where $\delta x$ is defined by Equation (74).

Thus, the particles in position 1 take energy from the wave, while the particles in position 2 transfer energy to the wave. The growth of the wave is possible only if there are more latter particles than the former. Let $F(\varepsilon, x)$ be an undisturbed particle distribution function. Then the instability condition is written as

$$
F\left(\varepsilon_{\text {res }}-\delta \varepsilon, x_{0}-\delta x\right)-F\left(\varepsilon_{\text {res }}+\delta \varepsilon, x_{0}+\delta x\right)<0 .
$$

Here, $\varepsilon_{\text {res }}$ is the resonant frequency. Expanding this inequality into a Taylor series at the point yields an instability condition in the form

$$
\left[\frac{\partial F}{\partial \varepsilon} \delta \varepsilon+\frac{\partial F}{\partial x} \delta x\right]_{\varepsilon_{\mathrm{res}}}>0 .
$$

Substituting $\delta \varepsilon$ and $\delta x$ from (73), (74), after some manipulations we obtain the instability condition in the form

$$
\left[\frac{\partial F}{\partial \varepsilon}+\frac{k_{y}}{\omega} \frac{c}{q B} \frac{\partial F}{\partial x}\right]_{\varepsilon_{\mathrm{res}}}>0 .
$$


In a curved magnetic field,

$$
\left[\frac{\partial F}{\partial \varepsilon}+\frac{m}{\omega} \frac{c}{q B_{\mathrm{eq}} L} \frac{\partial F}{\partial L}\right]_{\varepsilon_{\mathrm{res}}}>0
$$

The expression in square brackets can be written as

$$
\frac{d F}{d \varepsilon}=\frac{\partial F}{\partial \varepsilon}+\frac{\partial L}{\partial \varepsilon} \frac{\partial F}{\partial L}
$$

where

$$
\frac{\partial L}{\partial \varepsilon}=\frac{m}{\omega} \frac{c}{q B_{\mathrm{eq}} L} .
$$

The instability leading to the wave increase is seen to be possible in one of two cases. First, the distribution function increases with energy at a certain energy interval, and the resonant energy falls into this interval. Such distribution function can be called the inverted distribution function (the term bump on tail is often used). Secondly, there may be a strong distribution function spatial gradient for particles with resonant energy. The sign of the gradient must coincide with the azimuthal wave number sign. The corresponding instability can be coined as the gradient instability. The small azimuthal scale $(m>>1)$ is beneficial for this instability.

The case of curved field lines, when a particle experiences curvature drift, is treated similarly. The instability criterion is the same, (77) (refer to the original article [Huba and Drake, 1982]).

\subsection{Alfvén mode}

If the plasma pressure is small, in gyrokinetics, instead of Equation (48), the Alfvén mode is described by equation

$$
k_{1}^{2} \hat{L}_{\mathrm{T}}(\omega) \Phi+m^{2} \hat{L}_{\mathrm{P}}(\omega) \Phi+m^{2} \delta \hat{L}_{\mathrm{P}}(\omega) \Phi=0 .
$$

Here

$$
\begin{aligned}
& \delta \hat{L}_{\mathrm{P}}(\omega) \Phi=\frac{4 \pi \omega^{2}}{c^{2} M^{2}} \sqrt{g} \sum_{e, i} q\left\langle\frac{m \omega_{\mathrm{d}}}{\omega} K_{\mathrm{s}}\right\rangle, \\
& \langle\ldots\rangle=4 \pi \int(\ldots) \frac{B}{\left|v_{\|}\right|} d \mu d \varepsilon, \\
& K_{s}=q \hat{Q} F \frac{\omega}{\tau_{\mathrm{b}}} \times \\
& \times \sum_{K} \frac{\cos I_{l_{1}}^{l}}{\omega-m \bar{\omega}_{\mathrm{d}}-K \omega_{\mathrm{b}}} \int_{l_{1}}^{l} \frac{d l^{\prime}}{\left|v_{\|}\right|} \frac{m \omega_{\mathrm{d}}}{\omega} \Phi \cos I_{l_{1}}^{l^{\prime}},
\end{aligned}
$$

$l_{1}$ is the particle reflection point in the bounce motion,

$$
\begin{aligned}
& \hat{Q} F=\frac{\partial F}{\partial \varepsilon}+\frac{m}{\omega} \frac{c}{q B_{\mathrm{eq}} L} \frac{\partial F}{\partial L}, \\
& I_{a}^{b}=\int_{a}^{b} \frac{d l^{\prime}}{\left|v_{\|}\right|}\left(\omega-m \omega_{\mathrm{d}}\right) .
\end{aligned}
$$

Due to the presence of the resonant denominator $\omega-m \bar{\omega}_{\mathrm{d}}-K \omega_{\mathrm{b}}$, the third term of Equation (81) is complex. Hereinafter, the distribution function integral over the velocity space is assumed to be equal to the concentration of particles.

The real part of Equation (81) leads to an insignificant poloidal frequency correction and is not taken into account. The imaginary part occurs when bypassing the singularity according to the Landau rule:

$$
\frac{1}{\omega-m \bar{\omega}_{\mathrm{d}}-K \omega_{\mathrm{b}}} \rightarrow i \pi \delta\left(\omega-m \bar{\omega}_{\mathrm{d}}-K \omega_{\mathrm{b}}\right) .
$$

Since the third term in (81) includes the factor $m^{2}$, the existence of the imaginary part of this term will lead to the appearance of the imaginary part of the poloidal frequency $\operatorname{Im} \Omega_{\mathrm{PN}}$. If $\operatorname{Im} \Omega_{\mathrm{PN}}<0$, there is a collisionless damping of the wave due to the energy transfer from the wave to energetic particles. Otherwise, in the case $\operatorname{Im} \Omega_{\mathrm{PN}}>0$, the instability takes place. The instability growth rate caused by the resonant waveparticle interaction is

$$
\begin{aligned}
& \gamma=\sum_{K=-\infty}^{\infty} \gamma_{K}, \\
& \gamma_{K}=\frac{4 \pi}{c^{2}} \frac{q^{2}}{M^{2}} B_{\mathrm{eq}} L \times \\
& \times \int \hat{Q} F \omega_{\mathrm{b}} \delta\left(\omega-m \bar{\omega}_{\mathrm{d}}-K \omega_{\mathrm{b}}\right) G^{2}(\mu, \varepsilon) d \mu d \varepsilon, \\
& G(\mu, \varepsilon)=\int_{l_{1}}^{l_{2}}\left|v_{\|}\right|^{-1} \omega_{\mathrm{d}} P_{N} \cos I(l) d l,
\end{aligned}
$$

where $P_{N}$ is the poloidal operator eigenfunction normalized as

$$
\int_{l_{-}}^{l_{+}} \sqrt{\frac{g_{1}}{g_{2}}} \frac{P_{N}^{2}}{v_{\mathrm{A}}^{2}} d l=1
$$

[Karpman et al., 1977]. In agreement with the results of the previous section and as evident from Equation (88), the instability can be inverted $(\partial F / \partial \varepsilon>0)$ or gradient $\left(m \cdot \partial F / \partial x^{1}>0\right)$. The Alfvén wave inverted instability was first proposed by Korablev and Rudakov [1968]; the gradient instability, by Southwood et al. [1969]. If the bounce frequency is very high, only the term $K=0$ should be left in (87), which corresponds to the drift resonance. A more general case, when it is necessary to sum all the bounce harmonics, was studied in detail by Pilipenko et al. [1977]. The numerical simulation of Alfvén wave excitation in the drift-bounce resonance due to the gradient instability with the magnetospheric plasma azimuthal inhomogeneity taken into account was carried out by Yamakawa et al. [2020].

Note that the wave frequency $\omega$ in the resonant denominator $\left(\omega-m \bar{\omega}_{\mathrm{d}}-K \omega_{\mathrm{b}}\right)$ is not an arbitrary value. Since the increment is a correction to the poloidal eginefrequency $\Omega_{\mathrm{PN}}(L)$, the wave frequency should be identified with $\Omega_{\mathrm{PN}}(L)$. Thus, the frequency is defined by the $L$-shell, where the wave is localized. Since $\bar{\omega}_{\mathrm{d}}$ and $\omega_{\mathrm{b}}$ are the energy functions, the resonance condition determines the localization of the wave generated by the instability across magnetic shells at given values of the resonance energy $\varepsilon_{\text {res }}$ (for example, the energy corresponding to the inverted part of the 
distribution function), azimuthal wave number $m$, and bounce harmonic $K$ [Mager and Klimushkin, 2005].

The appearance of imaginary addition only to the poloidal frequency is due to Alfvén wave-particle interaction that occurs due to the poloidal mode electric field azimuthal component, in accordance with energy equation (17). Hence, it is often concluded that poloidal waves excite instabilities in the magnetosphere. However, this conclusion needs correction.

Let us turn to the case when the Alfvén wave propagates in the region of monotonic variation of toroidal and poloidal frequencies. Two limiting situations can be considered: a wave is generated by a monochromatic source or by an impulse one. In the former case, the wave propagates across magnetic shells, changing its polarization from poloidal to toroidal (see Subsection 3.3). At the same time, its amplitude increases due to interaction with energetic particles. As it propagates through the magnetosphere, the growth rate of the wave amplitude decreases and becomes zero when the wave reaches the toroidal surface (becomes toroidal). However, the cumulative effect of the amplitude increase due to instability takes place at the end of this process, when the wave has become toroidal (Figure 17) [Klimushkin, 2000]. If the wave is simultaneously damped due to the finite conductivity of the ionosphere, the maximum amplitude moves away from the toroidal surface toward the poloidal one. Nevertheless, the stronger the instability, the closer the wave amplitude maximum to the toroidal surface.

The same situation arises in the impulse-generated wave case. However, transformation occurs not due to propagation across magnetic shells, but due to phase mixing. The stronger the instability, the larger the amplitude part falls on the toroidal polarization (Figure 18) [Klimushkin and Mager, 2004].

Thus, in both the monochromatic and impulsegenerated wave cases, the instability ultimately generates a toroidal wave. The only way to circumvent this difficulty is to assume that in most cases poloidal waves are enclosed in a resonator across magnetic shells (see Subsection 3.4). It is the only case when the instability can generate a wave with predominantly poloidal polarization.

\subsection{Drift-compressional mode}

Compressional modes in the magnetosphere are observed as Pc5 pulsations with the longest periods (so called storm time compressional Pc5 waves). The association of these waves with storm activity periods indicates the importance of hot plasma in their generation. An interesting property of such waves is diamagnetism: the plasma and magnetic pressures oscillate in antiphase [Moiseev et al., 2016]. Among MHD waves, this property is attributed to SMS waves. However, as mentioned above, in kinetics the existence of SMS waves requires a pronounced parallel electric field of the wave. Since the presence of even a small admixture of cold electrons leads to zeroing of the parallel electric field, the possibility of the existence of

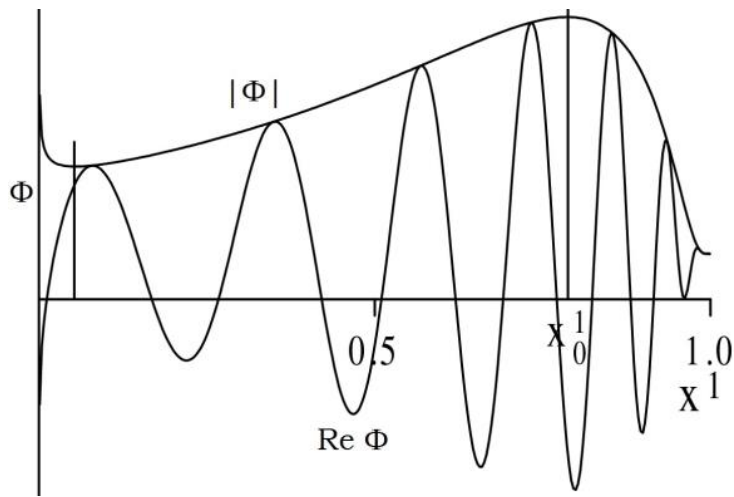

Figure 17. Wave amplitude distribution across field lines and the wave field structure in the case of a wave propagating across field lines and amplified due to instability [Klimushkin, 2000]. The points $x^{1}=0$ and $x^{1}=1$ correspond to the poloidal and toroidal surfaces respectively. The amplitude maximum position is marked with $x_{0}^{1}$

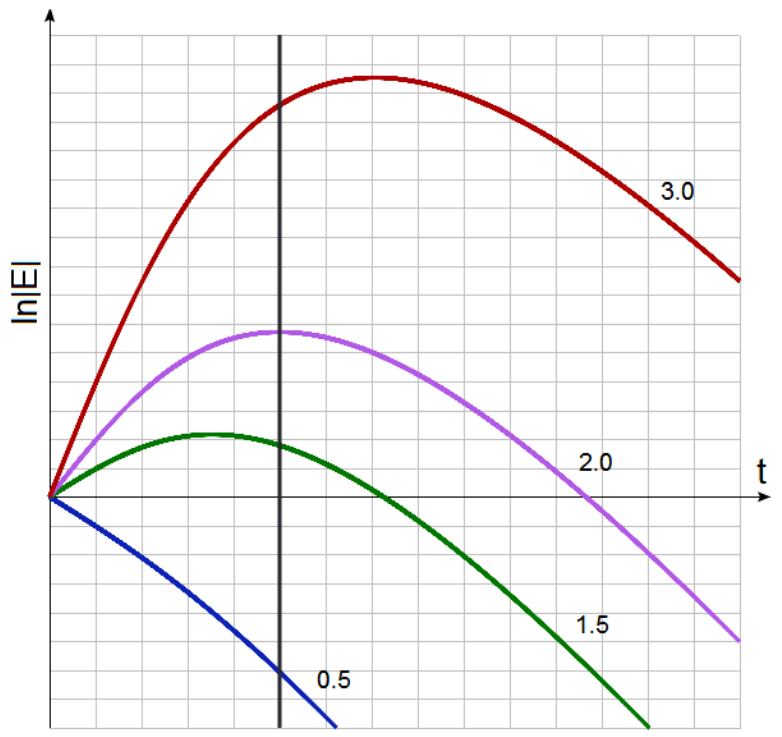

Figure 18. Time dependence of the wave amplitude logarithm $|E|$ for different ratios of the instability increment to the damping decrement (indicated near curves). On the vertical axis are conventional units (logarithmic scale). The plot is for $m=50$, the decrement is 10 times less than the toroidal frequency. The vertical line separates the regions of predominantly poloidal (to the left of it) and toroidal wave polarization

SMS waves in the magnetosphere within the framework of kinetics is questionable.

Nevertheless, the compressional ULF modes with diamagnetic properties can exist. Integrating the Vlasov equation readily yields force balance equation [Pokhotelov et al., 2000]:

$$
P+\frac{B_{0} B_{\|}}{4 \pi}=\frac{k_{\|}^{2}}{k_{\perp}^{2}}\left[\frac{\omega^{2}}{k_{\|}^{2} v_{\mathrm{A}}^{2}}-1\right] \frac{B_{0} B_{\|}}{4 \pi} .
$$

The azimuthally small-scale waves are characterized by the condition $k_{\|} \ll k_{\perp}$. Equality (91) is therefore reduced to diamagnetism condition

$$
P+\frac{B_{0} B_{\|}}{4 \pi} \simeq 0
$$


Thus, diamagnetism is not associated with specific wave types, but is a rather general property of azimuthal small-scale oscillations in finite-pressure plasma.

In some works, the compressional Pc5 waves are associated with one of the kinetic modes in inhomogeneous plasma, so-called drift-compressional modes [Ng and Patel, 1983; Migliuolo, 1983; Ng et al., 1984; Crabtree and Chen, 2004]. This mode is most easily illustrated in the cylindrical model of the magnetosphere. The magnetic field and plasma are considered to be one-dimensionally inhomogeneous. Field lines are concentric circles, and magnetic surfaces are nested coaxial cylinders. All equilibrium parameters depend only on the distance from the cylinder axis. Despite its simplicity, this model allows studying compressional waves in the magnetosphere since it takes into account such basic magnetosphere features as the field line curvature and the inhomogeneous plasma pressure profile. In this model, the dispersion equation for compressional modes has the form

$$
\begin{aligned}
& L_{\mathrm{M}}=-1+\frac{4 \pi \omega^{2}}{c^{2}} \frac{2 \pi}{k_{\perp}^{2}} \sum_{e, i} q^{2} \int d v_{\|} d v_{\perp} v_{\perp}^{3} J_{1}^{2}(\xi) \hat{Q} F \times \\
& \times\left[\omega-k_{y} u_{\mathrm{d}}-k_{\|} v_{\|}\right]^{-1}=0
\end{aligned}
$$

[Klimushkin and Mager, 2011], where $\xi=k_{\perp} v_{\perp} / \omega_{\mathrm{c}}$ is the Bessel function argument $J_{1}$,

$$
\hat{Q} F=\frac{\partial F}{\partial \varepsilon}+\frac{k_{y} c}{\omega} \frac{F^{\prime}}{q B_{0}} .
$$

Here, the prime means the derivative to the radial coordinate (the cylinder radius). Let us now consider the quasi-hydrodynamic limit when $\Omega_{\mathrm{d}} / \omega \ll 1, \quad \xi \ll 1$, $\omega, \Omega_{\mathrm{d}} \gg k_{\|} V_{\|}$. Then the dispersion equation is

$$
-1-\beta \frac{\omega-\omega_{*}}{\omega}+i \beta \Gamma_{\mathrm{M}}=0
$$

[Klimushkin and Mager, 2011]. Here,

$$
\begin{aligned}
& \tilde{\omega}_{*}=\frac{k_{y}}{\omega_{\mathrm{c}}} T^{\prime}, \\
& \omega_{*}=\frac{k_{y}}{\omega_{\mathrm{c}}} \frac{n^{\prime}}{n} T\left(1-\frac{3}{2} \eta\right), \\
& \eta=\frac{T^{\prime}}{n^{\prime}} \frac{n}{T}, \\
& \Gamma_{\mathrm{M}}=\frac{\sqrt{\pi}}{2} \int_{0}^{1} \frac{\lambda^{2} d \lambda}{\sqrt{1-\lambda}}\left(\frac{\omega-\omega_{*}}{\omega}-\frac{\tilde{\omega}_{*}}{\Omega_{\mathrm{d}}}\right)\left(\frac{\omega}{\Omega_{\mathrm{d}}}\right)^{\frac{7}{2}} e^{-\frac{\omega}{\Omega_{\mathrm{d}}}} .
\end{aligned}
$$

The dispersion relation produces a wave frequency real part and an instability growth rate

$$
\begin{aligned}
& \omega=\frac{\omega_{*} \beta}{1+\beta}, \\
& \gamma_{M}=\omega_{*} \frac{\beta^{2}}{(1+\beta)^{2}} \Gamma_{M} .
\end{aligned}
$$

Thus, the wave frequency is of the order of the diamagnetic drift frequency (when $\beta \sim 1$ ). As follows from (101), a necessary instability condition is an increase in the average particle energy with distance from Earth. A large value of $\beta$ is beneficial for the instability.

The situation is more complicated in plasma with a field aligned inhomogeneity when the bounce motion of particles is taken into account. Since the observed compressional wave frequencies are much lower than the bounce frequencies of energetic particles of the ring current and radiation belts, the greatest contribution in the sum over bounce harmonics is made by the term with $K=0$ corresponding to the drift resonance. In this case, the wave parallel structure is described by the integral (rather than differential, as in the Alfvén wave case) equation [ $\mathrm{Ng}$ et al., 1984; Crabtree et al., 2003; Crabtree and Chen, 2004]. The drift-compressional mode was found to be narrowly localized near the geomagnetic equator, in the region of maximum $\beta$ (Figure 19). This is consistent with the observational data on Pc5 compressional waves [Takahashi et al., 1987]. The mode eigenfrequency is [Mager et al., 2013]

$$
\Omega_{\mathrm{M}}=\frac{m}{L} \frac{\frac{2}{3} \frac{L_{\mathrm{b}}}{\beta_{\mathrm{eq}}} \Lambda_{N} V_{\mathrm{d}}+V_{n}^{*}+V_{T}^{*}}{1-\frac{V_{T}^{*}}{V_{\mathrm{d}}}} .
$$

Here, $L_{\mathrm{b}}$ is the particle trajectory length along the field line between the reflection points, $\beta_{\mathrm{eq}}$ is the plasma pressure to magnetic pressure ratio at the equator, $V_{\mathrm{d}}$ is the drift velocity in the inhomogeneous magnetic field, $V_{n}^{*}$ and $V_{T}^{*}$ are the diamagnetic drift velocities due to concentration and temperature gradients respectively. The values $\Lambda_{N}$ characterize the wave eigenfrequencies. For three main harmonics, they are equal to $\Lambda_{1}=0.5 / R_{\mathrm{eq}}$, $\Lambda_{2}=1.5 / R_{\text {eq }}, \Lambda_{3}=2.5 / R_{\text {eq }}$, where $R_{\text {eq }}$ is the field line curvature equatorial radius. The drift-compressional mode frequency is seen to linearly depend on the azimuthal wave number $m$. More detailed study of the structure and excitation conditions of the driftcompressional modes was carried out by Mager et al. [2013]. It was found that wave excitation due to drift resonance with energetic protons occurs when one of two conditions is met: either the plasma temperature increases with distance from Earth or an inverted distribution takes place in a certain region of energies: a bump on tail distribution function. The wave propagates westward, in the proton drift direction (negative wave numbers, $m<0)$. However, in the presence of hot electrons in plasma, the drift-compressional waves propagating in the electron drift direction (to the east, $m>0$ ) may exist [Kostarev and Mager, 2017].

\subsection{Coupling of Alfvén and drift- compressional modes}

Alfvén and drift-compressional modes are coupled due to the plasma and magnetic field inhomogeneity in a finite pressure plasma. The mode coupling effect on the wave generation conditions in a one-dimensional inhomogeneous magnetosphere model with straight field lines was studied by $\mathrm{Ng}$ and Patel [1983]; in the 


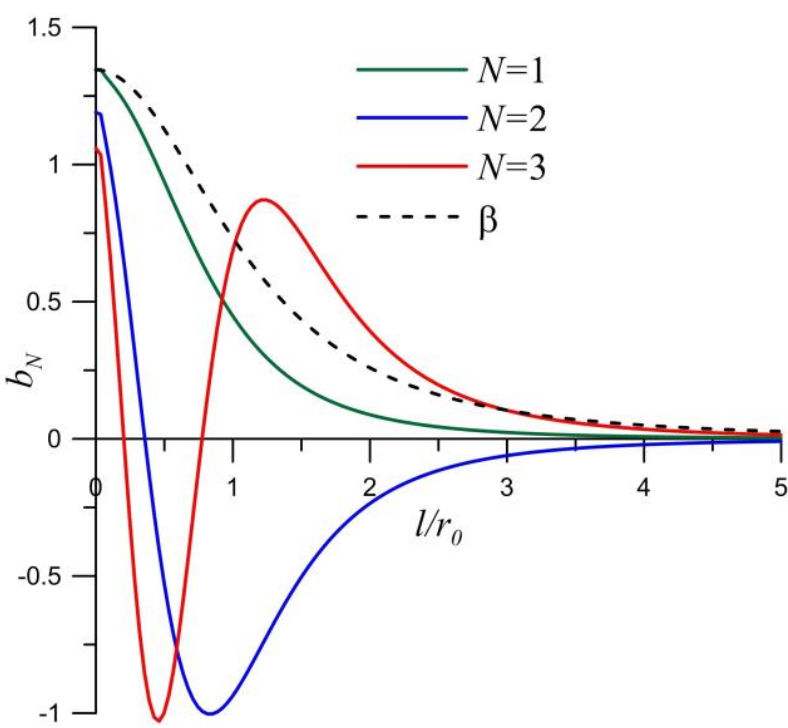

Figure 19. Parallel structure $b_{N}$ of the first three harmonics of the drift-compressional mode [Mager et al., 2013]. The dotted line shows the behavior of $\beta$ along a field line

cylindrical model, by Klimushkin et al. [2012]; and in the dipole-like (two-dimensionally inhomogeneous model), by Mager and Klimushkin [2017]. It has been found that for certain parameters the coupling gives rise to a new plasma instability, a kinetic analogy of ballooning instability in MHD.

As shown by Mager and Klimushkin [2017], the instability is possible when the wave frequency is much higher than the average drift frequency of hot protons. In this case, the wave behaves as follows (Figure 20). At small azimuthal wave numbers $m$, the poloidal Alfvén and drift compressional modes represent two separate oscillation branches. In this case, the Alfvén wave frequency $\Omega_{\mathrm{P}}$ exceeds the drift compressional mode frequency $\Omega_{\mathrm{M}}$. However, $\Omega_{\mathrm{M}}$ grows almost linearly with $m$, whereas $\Omega_{\mathrm{P}}$ slowly decreases with increasing $m$. At a certain critical azimuthal wave number value $m_{\mathrm{c}}$, frequencies of these two branches become equal, the branches merge and form two new modes, also known as drift-coupling modes. The real parts of the frequency for both coupling modes are the same, but the sign of the imaginary parts are opposite: one of the coupling modes is damped, the other is unstable. With a further increase in the azimuthal wave number, the stable and unstable coupling modes merge again, forming the Alfvén and drift-compressional modes. Nonetheless, the Alfvén wave frequency here is lower than that of the drift-compressional mode.

\subsection{Drift-mirror mode}

The drift-compressional mode is the simplest compressional mode in an inhomogeneous plasma since its existence requires only a finite plasma pressure and plasma inhomogeneity. Along with it, one more compressional mode is widely discussed in magnetospheric physics, the drift-mirror mode. Its existence requires plasma pressure anisotropy (inequality

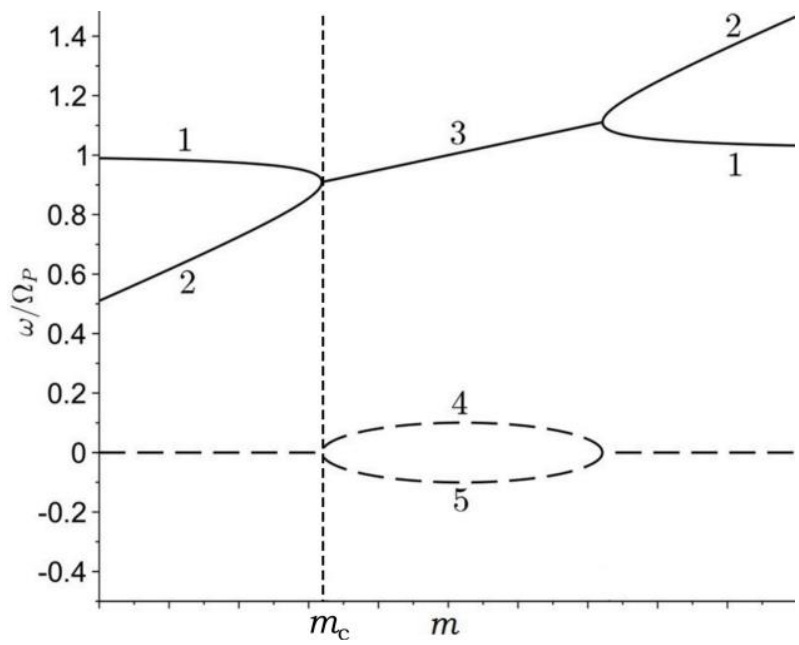

Figure 20. Frequency dependence of coupled Alfvén and drift-compressional modes on the azimuthal wave number [Mager and Klimushkin, 2017]: 1 is an Alfvén mode; 2 is a drift-compressional mode; 3 is a drift-coupling mode, the real part of the frequency; 4 and 5 , a drift-coupling mode, the imaginary part of the frequency; 4 is an increasing mode; 5 is a decreasing mode

of longitudinal and transverse pressures).

To study this mode, return to dispersion equation (95), but consider the opposite limit $k_{\|} v_{\|} \gg \omega, \omega_{\mathrm{d}}$, sometimes called the mirror approximation. Then, the resonance term in (94) is rewritten as $\left(\omega-k_{y} u_{\mathrm{d}}-k_{\|} v_{\|}\right)^{-1} \approx-\frac{i \pi}{k_{\|}} \delta\left(v_{\|}\right)$. In this limit, Equation (95) is reduced to the form

$$
\tau-i \frac{\omega-\omega_{*}}{\Gamma}=0 .
$$

Here,

$$
\begin{aligned}
& \tau=1+\beta_{\perp}\left(1-\frac{\beta_{\perp}}{\beta_{\|}}\right), \\
& \omega_{*}=\frac{k_{y} V_{\|}^{2}}{\omega_{\mathrm{c}}}\left[\frac{n^{\prime}}{n}+3 \frac{T_{\|}-T_{\perp}}{T_{\|}} \frac{B^{\prime}}{B}\right], \\
& \Gamma=\sqrt{\frac{2}{\pi}} \frac{T_{\|}}{T_{\perp}} \frac{k_{\|} V_{\|}}{\beta_{\perp}}
\end{aligned}
$$

The mode determined by dispersion equation (103) is called the drift-mirror mode. It was first obtained by Hasegawa [1969]. The real and imaginary parts of this mode frequency are

$$
\begin{aligned}
& \operatorname{Re} \omega=\omega_{*}, \\
& \gamma=-\tau \Gamma
\end{aligned}
$$

(the correct expression for the real part of the frequency was first obtained by Pokhotelov and Pilipenko [1976]). When the condition $\tau<0$ holds, a so-called mirror instability arises. Write down the instability-condition explicitly:

$$
\frac{\beta_{\perp}}{\beta_{\|}}>1+\frac{1}{\beta_{\perp}} .
$$


It is seen that for the development of this instability the transverse pressure must exceed the parallel. Moreover, the instability threshold decreases with increasing pressure. The finite electron pressure and the finite Larmor radius of ions significantly influence the mirror instability development [Pokhotelov et al., 2000; Pokhotelov et al., 2005; Klimushkin and Chen, 2006; Feygin and Khabazin, 2014].

For $\beta \sim 1$, coupling with the Alfvén mode greatly affects the mirror instability [Lin and Parks, 1978; Migliuolo, 1983; Pokhotelov et al., 1985; Woch et al., 1988]. In this case, the instability growth rate, instead of (108), takes the form

$$
\gamma=-\Gamma \tau+\Gamma \frac{\beta_{\perp}^{2}}{4} \frac{1}{k_{\|}^{2}}\left(\frac{P_{\perp}^{\prime}}{P_{\perp}}\right)^{2}\left(\sigma+\frac{\Gamma^{2} \tau^{2}}{k_{\|}^{2} v_{\mathrm{A}}^{2}}\right)^{-1}
$$

where $\sigma=1+1 / 2\left(\beta_{\perp}-\beta_{\|}\right)$[Klimushkin and Mager, 2012]. As seen from (110), when the coupling is taken into account, the mirror instability is possible even for positive $\tau$ value. If there are no cold electrons in the plasma, dispersion relation (103) is replaced by

$$
\omega^{2}=k_{\|}^{2} \frac{v_{i}^{2} \tau v_{\mathrm{A}}^{2}}{v_{\mathrm{S}}^{2}+\tau v_{\mathrm{A}}^{2}},
$$

where $v_{i}=V_{\| \mathrm{eh}} \sqrt{m_{\mathrm{e}} / m_{\mathrm{i}}}$ is the ion sound velocity, $V_{\| \text {eh }}$ is the parallel thermal velocity of electrons [Klimushkin and Kostarev, 2012]. In the isotropic pressure case $(\tau=1)$, this relation coincides with dispersion relation of slow magnetic sound (45). When $\tau<0$, the square of the frequency becomes negative, i.e. one of the dispersion equation roots corresponds to instability. This instability may be called quasi-hydrodynamic mirror instability, in contrast to the kinetic mirror by Hasegawa [1969]. The threshold of these two mirror instability types coincides $(\tau<0)$, but their character is much different. If the instability condition is not met, then the kinetic mirror mode is damped, while mode (111) oscillates, like a slow magnetoacoustic wave. Since there is always a certain fraction of cold electrons in the magnetosphere, the quasihydrodynamic mirror instability most likely cannot exist there, yet it can exist in the transition layer.

In contrast to the drift compressional mode, the theory of drift-mirror instability was developed only for a longitudinally homogeneous plasma. Several attempts have been made to extend this theory to the dipole-like magnetic field case [Pilipenko and Pokhotelov, 1977; Cheng and Lin, 1987; Cheng and Qian, 1994]. However, none of them dealt with the possibility of changing the sign of $\tau$ along the field line. Near the equator, the mirror instability condition $\tau<0$ is satisfied. Nonetheless, when moving along field lines, the value of $\tau$ inevitably passes through zero, then becomes positive, and rapidly tends to unity (Figure 21). Indeed, the plasma pressure is constant along the field line, while the magnetic pressure increases due to the convergence of field lines near the ionosphere. The properties of oscillation modes that may exist in this case have not been studied yet. Until this problem is being solved, it seems impossible to talk about the possibility of mirror mode existence in the magnetosphere.

\section{EXPERIMENTAL DATA ON SMALL-SCALE AZIMUTHAL WAVE GENERATION BY INSTABILITIES}

It is currently assumed that instabilities associated with the wave-particle energy transfer generate azimuthally small-scale ULF waves, mostly poloidal or compressional. Some evidence argues in favor of generation of azimuthally small-scale waves by energetic particles, especially protons. The evidence includes the statistical correlation between observations of these waves and intensifications of the ring current [Anderson, 1993; Yeoman et al., 2000] and the similarity between the spatial distribution of these waves and proton drift trajectories [Takahashi, 1996].

The shielding effect of the atmosphere prevents the electromagnetic field of these waves from penetration to Earth's surface (the exception is the so-called giant pulsations (Pgs), which have moderately large azimuthal wave numbers $m \simeq 20$ ). Standard techniques for observing azimuthally small-scale waves involve spacecraft and radars. The first examples of waves with $m>>1$ observed using these methods were given respectively in [Cummings et al., 1969] and [Allan et al., 1982; Walker et al., 1982]. Other methods for studying these waves include pulsations of the riometric absorption [Beharrell et al., 2010; Moiseev et al., 2020], the ionospheric total electron content measured with Global Navigation Satellite System receivers [Watson et al., 2016; Zhai et al., 2021], and optical data [Motoba et al., 2015; Baddeley et al., 2017].

\subsection{Poloidal Alfvén modes}

To determine the type of instability generating a wave in a particular case, it is necessary to answer the following questions: (1) whether the observed mode is Alfvén, drift compressional, or drift mirror; (2) whether this mode symmetric or antisymmetric about the equator; (3) which particles (protons or electrons) generate the wave; (4) what is the energy range of these particles; (5) whether it is drift or drift-bounce resonance; (6) what is the character of the instability: the inverted distribution function or its spatial gradient. Signatures of an Alfvén wave include relatively high frequency and a regular, quasi-sinusoidal form of pulsations since the frequency of an Alfvén wave is determined mainly by its magnetic shell $L$. Another observational aspect of the Alfvén mode is a small (although not necessarily evanescently small) compressional component of the magnetic field. The first example of a wave with the resolved question about

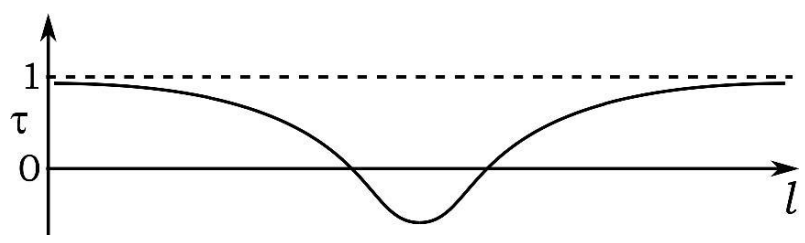

Figure 21. Parameter $\tau$ as a function of length along the field line $l$ 
the nature of the instability might have been found by Hughes et al. [1978]. The second harmonic $(N=2)$ of a standing wave was observed, which excludes the possibility of the drift resonance. The wave generation is likely to be related to the drift-bounce resonance with $K=1$. The proton distribution function $F(\varepsilon)$ featured a clear inverse segment at the 1 to $10 \mathrm{keV}$ energy range. A number of later works present other examples of the Alfvén waves with $N=2$ in the drift-bounce resonance with 1-10 keV protons with the inverse distribution function [Hughes et al., 1979; Wright et al., 2001; Baddeley et al., 2002; Liu et al., 2013; Takahashi et al., 2018b].

This mechanism does seem to be quite common. Statistical research by Baddeley et al. [2004] and Baddeley et al. [2005] show that in most cases the inverse instability of protons in the $10-45 \mathrm{keV}$ energy range is responsible for the generation of poloidal Alfvén waves with $m>>1$. The resonance is driftbounce, and Alfvén waves have $N=2$. The free energy of particles in this range exceeds $10^{10} \mathrm{~J}$. Besides, the populations of particles observed simultaneously with poloidal Alfvén waves have higher free energy than that in average conditions.

There are, however, cases not consistent with this scenario. A case where the fundamental harmonic was in the drift resonance with of $80 \mathrm{keV}$ protons is described by Mager et al. [2018]. The wave was generated by the inverse instability (Figure 22). It was observed in the region of the poloidal frequency maximum at the outer edge of the plasmapause. The oscillation is likely to be a superposition of several (at least two) harmonics of the transverse Alfvén resonator, which was discussed in Subsection 3.4. Of particular note is also the case from [Glassmeier et al., 1999]. A poloidal wave was observed simultaneously with an inverse distribution function of protons with energies of about $60 \mathrm{keV}$. The most interesting detail is that for such parameters drift-bounce resonance condition (20) gives a non-integer bounce harmonic number $K$. The authors attribute this to the asymmetry of the wave relative to the equator. This is possible if the boundary conditions at the southern and northern points of intersection of a field line with the ionosphere are contrastingly different. This assumption sparked a discussion [Mann and Chisham, 2000; Glassmeier, 2000], which is however beyond the scope of this review.

The inverse instability hypothesis is supported by the fact that poloidal Alfvén waves are often registered simultaneously with substorm-injected particles reaching an azimuth of the observation point [Chisham et al., 1992; Wright et al., 2001]. Indeed, the dispersion of drift velocities of particles entering the magnetosphere in a single injection is among the inverse energy distribution formation mechanisms, as it was mentioned in Subsection 4.1. However, we should be cautious in this case. Simultaneous onset of pulsations and injected particles may be a manifestation of a completely different wave generation mechanism: a moving source (Subsection 3.5). These two mechanisms are especially easy to confuse since the azimuthal wave number of an oscillation excited by a moving source also satisfy the drift resonance condition, although its physical meaning is different.

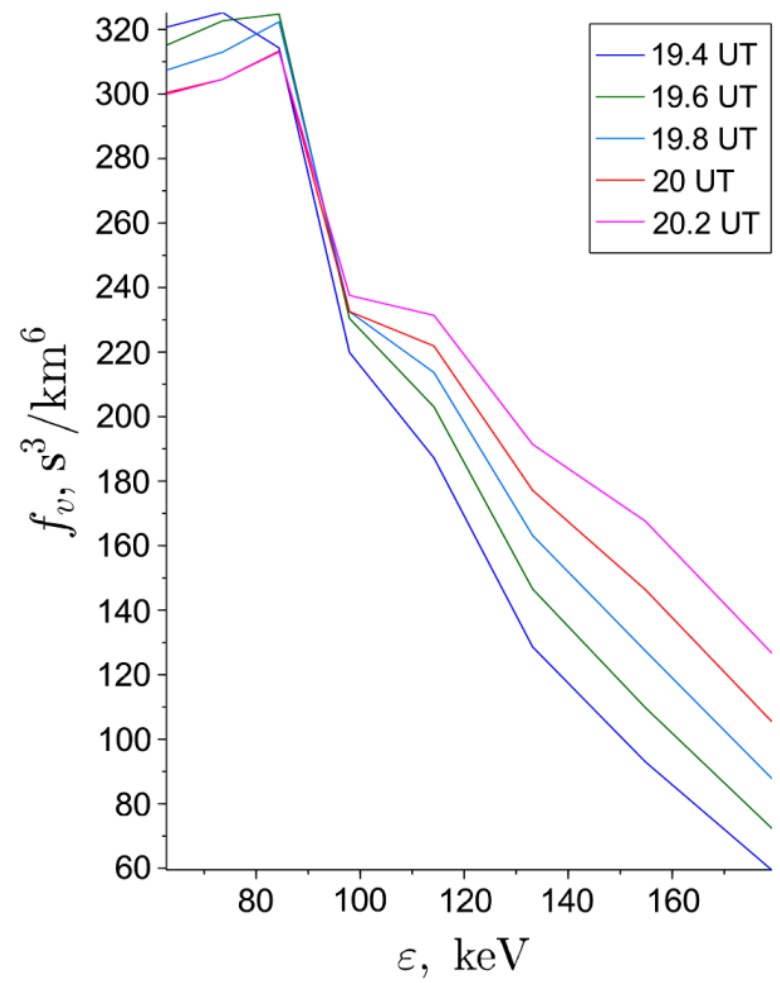

Figure 22. Inverse proton distribution function for a case described in [Mager et al., 2018]

A number of examples of Alfvén waves generated by the gradient instability have been found relatively recently. Dai et al. [2013] and Takahashi et al. [2018a] studied fundamental harmonics $(N=1)$ generated by drift resonance with protons with energies of about 90 and $140 \mathrm{keV}$ respectively. Min et al. [2017], Oimatsu et al. [2018], Mager [2021], Rubtsov et al. [2021] examined the second harmonic of standing waves $(N=2)$ that were in the drift-bounce resonance with protons of 80,120 180 , and $46 \mathrm{keV}$ respectively. In all these four cases, the waves propagated westward, in the proton drift direction, like the majority of azimuthal small-scale waves. However, an eastward-propagating wave $(N=2)$ was studied by Yamamoto et al. [2019]. Propagating in the electron drift direction, the wave was, nevertheless, in the drift-bounce resonance with the 10-30 keV proton population that formed a gradient instability. Finally, an interesting example of an Alfvén wave was examined by Wei et al. [2019]: both inverse and gradient instabilities were responsible for the mode excitation at different time intervals. A second harmonic was observed with drift-bounce resonance and resonance energy of protons of about $10 \mathrm{keV}$.

\subsection{Drift compressional modes}

As we mentioned above, drift compressional modes are the most typical compressional modes in magnetospheric plasma since their existence requires only a finite plasma pressure and plasma inhomogeneity. A distinctive feature of this mode is a large parallel component of the magnetic field (comparable to or greater than the transverse components). The total magnetic field and plasma pressure oscillate in antiphase (diamagnetism). Since 
the frequency of the drift compressional mode depends not only on the L-shell, but also on the azimuthal wave number, we should expect its spectrum to be wider than those of Alfvén waves. Its frequency can be significantly lower than the Alfvén frequency at the same magnetic shell. Such waves should be narrowly localized around the geomagnetic equator.

The storm time compressional Pc5 waves first distinguished by Barfield and McPherron [1972] fit these properties. Irregular Ps6 or Pi3 pulsations might also have this nature [Vaivads et al., 2001; Yagova et al., 2021]. The Pc5 pulsations have the longest periods of all ULF oscillations. They exhibit a diamagnetic property and often feature a non-sinusoidal profile with localization near the equator [Takahashi et al., 1987; Higuchi and Kokubun, 1988]. Thus, it seems reasonable to identify these waves with the drift compressional mode [Crabtree and Chen, 2004; Mager et al., 2013].

There are more compelling reasons for this interpretation. Chelpanov et al. [2016] studied a Pc5 pulsation with varying frequency and azimuthal wave number. The frequency depended on the $m$ number approximately linearly (Figure 23), as expected for the drift compressional modes. As shown by Chelpanov et al. [2018], such modes can be typical for the night-time magnetosphere: frequencies of Pc5 pulsations are generally several times lower than Alfvén frequencies at the same $L$-shells.

There is experimental evidence for the generation of the drift compressional modes by the gradient instability. As mentioned above, this requires an increase in the average energy of protons with distance from Earth. Such behavior was found for compressional Pc5 pulsations studied in [Rubtsov et al., 2018; Mager et al., 2019]. In both cases, frequencies of the observed waves were significantly lower than Alfvén frequencies at the magnetic shells where the oscillations were registered. This provides additional basis for identifying these waves as drift compressional modes.

The possibility of generating ULF waves due to the coupling of the Alfvén and drift compressional modes was shown by Mager et al. [2015]. These authors observed two modes with different frequencies and azimuthal wave numbers simultaneously, using a radar. When $|m|$ was below 30 , these modes with the same azimuthal wave number were discernible. The higher frequency almost did not depend on the $m$ value, whereas the lower one increased with $m$. As the azimuthal wave numbers exceeded 30, these two branches merged, and a single mode was observed. This behavior is peculiar for the coupled Alfvén and drift compressional modes (Subsection 4.4).

\subsection{Drift-mirror modes}

Early experimental studies of magnetospheric compressional waves, when the drift compressional mode was not yet known, usually dealt with the driftmirror mode [Lanzerotti et al., 1969; Woch et al., 1990]. However, for this mode, in addition to the finite pressure and plasma inhomogeneity, one more condition is required: the transverse plasma pressure should be significantly higher than the parallel one (the $\tau$ parameter should be negative). Indeed, several examples of Pc5 compressional waves satisfying this criterion have been found [Rae et al., 2007; Tian et al., 2020; Cooper et al., 2021]. Yet one should be cautious of identifying the observed waves with drift-mirror modes since the entire theory of these modes was developed for the case of longitudinally homogeneous plasma. A more realistic dipole case involves a problem of changing the sign of the $\tau$ parameter along a field line, which we discuss in the latter part of Subsection 4.5. The lack of theory that takes this factor into account limits further discussion of the drift-mirror mode existence in Earth's magnetosphere. On the other hand, pressure anisotropy can be an additional factor contributing to the drift compressional mode generation.

\section{MODULATION OF CHARGED PARTICLE FLUXES}

In the case of resonant interaction of waves with energetic particles, the distribution function of these particles is modulated (perturbed) in its rather narrow part near the resonance energy in a special way. The differential particle flux $J(\varepsilon, \alpha, \vec{r})$ is usually measured by satellites, i.e. the particle flux through a unit area at a given energy $\varepsilon$, pitch angle $\alpha$ and position in space $\vec{r}$. The differential flux is related to the velocitydistribution function (phase space distribution, PSD) $F(\vec{v}, \vec{r})=F(\varepsilon, \alpha, \vec{r})$ by simple relation

$$
J(\varepsilon, \alpha, \vec{r})=\frac{v^{2}}{M} F(\varepsilon, \alpha, \vec{r}) .
$$

Besides the differential flux $J$, the differential energy flux $J_{\varepsilon}=\varepsilon J$ and the omnidirectional differential flux, i.e. the flux integrated over the entire solid angle $\Omega$, are often used. In space physics, the following units of measurement of the listed quantities are usually applied: $\mathrm{s}^{3} / \mathrm{km}^{6}$ for the velocity distribution function $F$; $1 /\left(\mathrm{s} \cdot \mathrm{cm}^{2} \cdot \mathrm{sr} \cdot \mathrm{keV}\right)$ for the differential particle flux; and $\mathrm{keV} /\left(\mathrm{s} \cdot \mathrm{cm}^{2} \cdot \mathrm{sr} \cdot \mathrm{keV}\right)$ for the differential energy flux.

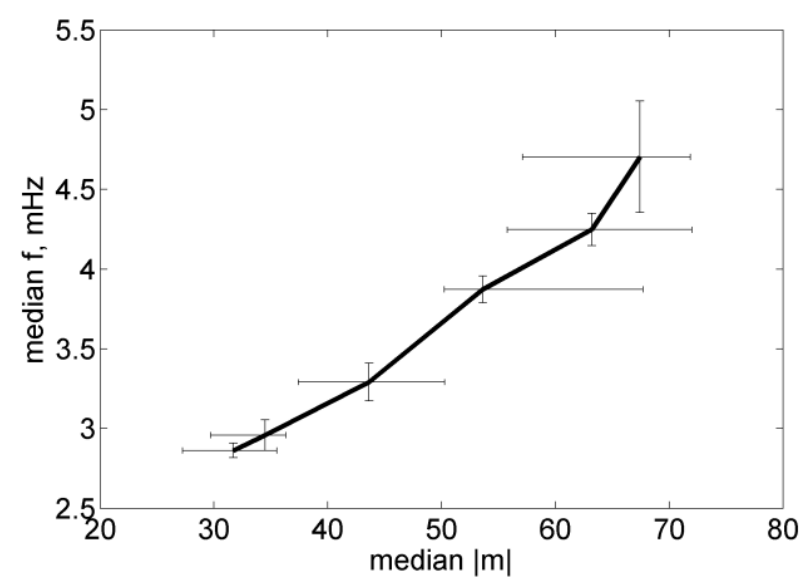

Figure 23. Frequency versus azimuthal wave number for the event described in [Chelpanov et al., 2016] 
Let $\delta F$ and $\delta J$ be the perturbations of the distribution function and flow caused by the particle-wave interaction, and $F$ and $J$ be their unperturbed values. In practice, according to satellite data, $\delta J$ and $J$ are separated from the observed flow by filtering as slow and fast components. To analyze the flux modulation by a wave, it is convenient to use the ratio $\delta J / J=\delta F / F$.

According to the theory developed by Chen and Hasegawa [1991], within the gyrokinetic framework the perturbation of the distribution function can be represented as

$$
\delta F=\delta F_{\mathrm{a}}+\delta K \exp \left(-\vec{k}_{\perp} \vec{\rho}\right),
$$

where the term with $\delta K$ is the resonant perturbation, $\delta F_{\text {a }}$ is the nonresonant (adiabatic) distribution function perturbation, and $\vec{\rho}$ is the particle gyroradius. In the vicinity of resonance energy $\varepsilon_{\mathrm{r}}$, the resonant perturbation significantly exceeds the adiabatic one, and we can assume that

$$
\delta F \approx \delta K \exp \left(-\overrightarrow{k_{\perp}} \vec{\rho}\right),
$$

where the term with $\delta K$ satisfies gyrokinetic equation

$$
\left[v_{\|} \frac{\partial}{\partial l}-i\left(\omega-m \omega_{\mathrm{d}}\right)\right] \delta K=i \delta S \hat{Q} F,
$$

where

$$
\delta S=m \omega_{\mathrm{d}} q \Phi+\omega \mu B_{\|},
$$

and

$$
\hat{Q} F=\frac{\partial F_{0}}{\partial \varepsilon}+\frac{m}{\omega} \frac{c}{q B_{\mathrm{eq}} L} \frac{\partial F}{\partial L} .
$$

Here, Equation (115) is written in approximation $k_{\perp} \rho \ll 1$, i.e. the transverse wavelength is considered to be much larger than the particle gyroradius. Besides, it is assumed that the parallel electric field of the wave is absent.

A complete solution of Equation (115) is given in [Chen and Hasegawa, 1991]. Let us present an approximate solution valid in the vicinity of the resonance, $\omega \approx m \bar{\omega}_{\mathrm{d}}+K \omega_{\mathrm{b}}$ :

$$
\delta K(\hat{\sigma})=\delta K_{\mathrm{s}}+\hat{\sigma} \delta K_{\mathrm{d}},
$$

where $\hat{\sigma}=v_{\|} /\left|v_{\|}\right|$,

$$
\begin{aligned}
& \delta K_{\mathrm{s}}=-\hat{Q} F \frac{\cos I_{l_{1}}^{l} \overline{\delta S \cos I_{l_{1}}^{l}}}{\omega-m \bar{\omega}_{\mathrm{d}}-K \omega_{\mathrm{b}}}, \\
& \delta K_{\mathrm{d}}=-i \hat{Q} F \frac{\sin I_{l_{1}}^{l} \overline{\delta S \cos I_{l_{1}}^{l}}}{\omega-m \bar{\omega}_{\mathrm{d}}-K \omega_{\mathrm{b}}},
\end{aligned}
$$

the bar denotes averaging over the bounce period

$$
\overline{(\ldots)}=\frac{2}{\tau_{\mathrm{b}}} \int_{l_{1}}^{l_{2}}(\ldots) \frac{d l}{\left|v_{\|}\right|},
$$

$l_{1}$ and $l_{2}$ are the reflection points of a particle during bounce motion,

$$
I_{l_{1}}^{l}=\int_{l_{1}}^{l} \frac{d l^{\prime}}{\left|v_{\|}\right|}\left(\omega-m \omega_{\mathrm{d}}\right) .
$$

Let us single out two main cases of wave-particle resonance: the drift resonance with the fundamental longitudinal harmonic of the wave, which has a distribution of the transverse potential $\Phi$ symmetric about the equator, and the drift-bounce resonance at $K= \pm 1$ with the second harmonic asymmetric about the equator.

\subsection{Drift resonance}

For the drift resonance with the fundamental harmonic, the particle flux modulation or the distribution function near the geomagnetic equator $(l=0)$ is determined by expression

$$
\frac{\delta J}{J}=\frac{\delta F}{F}=-\frac{\overline{\delta S}}{\omega-m \bar{\omega}_{\mathrm{d}}} \frac{\hat{Q} F}{F} \exp \left(-\vec{k}_{\perp} \vec{\rho}\right),
$$

It follows from this expression that the maximum amplitude of oscillations of the particle flux $\delta J$ is achieved at $m \bar{\omega}_{\mathrm{d}}=\omega$, i.e. at a certain resonant pair of values of energy $\varepsilon_{\text {res }}$ and pitch angle $\alpha_{\text {res }}$. This is because the angular drift velocity of the particles $\bar{\omega}_{\mathrm{d}}$ depends on the energy and the pitch angle of the particles (see (15)). Note that for fixed $\alpha=\alpha_{\text {res }}$, the $\delta J$ flux oscillations at energies above and below the resonant energy $\varepsilon_{\text {res }}$ occur in antiphase since, when passing through the resonant energy $\varepsilon_{\text {res }}$, the denominator $\omega-m \bar{\omega}_{\mathrm{d}}$ in expression (123) changes sign. Similarly, if we fix the energy $\varepsilon=\varepsilon_{\text {res }}$, the flux oscillation phase is changed by $180^{\circ}$ when passing through the resonant pitch angle $\alpha_{\text {res }}$

In practice, it is most useful to know the phase relationships between oscillations of the wave electric or magnetic field and oscillations of the particle flux at different energies and pitch angles. This allows us to determine the resonant energy of particles. Suppose that the wave parallel magnetic field is small or absent. This corresponds to Alfvén waves in most cases. Let the particles interacting with the wave be protons, then $\omega_{\mathrm{d}}<0$, and hence $m<0$. We also assume that $\omega=\omega_{0}+i \gamma$, where $\omega_{0}$ is the wave frequency, and $\gamma>0$ is the instability growth rate. Then, in the instability case the condition $\hat{Q} F>0$ holds (see Subsection 4.1). The azimuthal component of the Alfvén wave electric field is $E_{\mathrm{a}} \propto-i m \Phi$, hence

$$
\delta J \propto \frac{i E_{\mathrm{a}}}{\omega-m \bar{\omega}_{\mathrm{d}}} \exp \left(-\vec{k}_{\perp} \vec{\rho}\right) .
$$

Thus, the phase difference $\Delta \phi$ between $E_{\mathrm{a}}$ and $\delta J$ at a fixed pitch angle $\alpha$ varies depending on the particle energy as

$$
\Delta \phi= \begin{cases}\pi / 2+\vec{k}_{\perp} \vec{\rho} & \varepsilon>\varepsilon_{\mathrm{res}} \\ \vec{k}_{\perp} \vec{\rho} & \varepsilon=\varepsilon_{\mathrm{res}} \\ -\pi / 2+\vec{k}_{\perp} \vec{\rho} & \varepsilon<\varepsilon_{\mathrm{res}} .\end{cases}
$$

If we do not take into account the small correction due to the finite $\vec{k}_{\perp} \vec{\rho}$ value, at resonance energy $\varepsilon_{\text {res }}$ 
the particle flux oscillations are in phase with the wave electric field azimuthal component $E_{\mathrm{a}}$. This phase behavior is a hallmark of drift resonance. Knowing the phase relations between $E_{\mathrm{a}}$ and $\delta J$ (125), it is easy to find the same relations with the wave magnetic field radial component $B_{\mathrm{r}}$ and $\delta J$. At resonance energy $\varepsilon_{\text {res }}$, the phase difference between $B_{\mathrm{r}}$ and $\delta J$ is $\Delta \phi= \pm \pi / 2+\overrightarrow{k_{\perp}} \vec{\rho}$. The sign depends on the location of the magnetic field measurement relative to the magnetic equator since $B_{\mathrm{r}}$ is asymmetric about the equator, unlike $E_{\mathrm{a}}$. Note that in the case of the drift resonance with energetic electrons, phase relations (125) remain the same. However, for electrons $\omega_{\mathrm{d}}>0$, and therefore the drift resonance is possible for waves with $m>0$.

An example of the modulation of proton fluxes at the drift resonance with a wave, with the amplitude and phase behavior corresponding to the drift resonance, is given in Figure 24. Similar examples can also be found in [Dai et al., 2013; Rubtsov et al., 2018; Takahashi et al., 2018a].
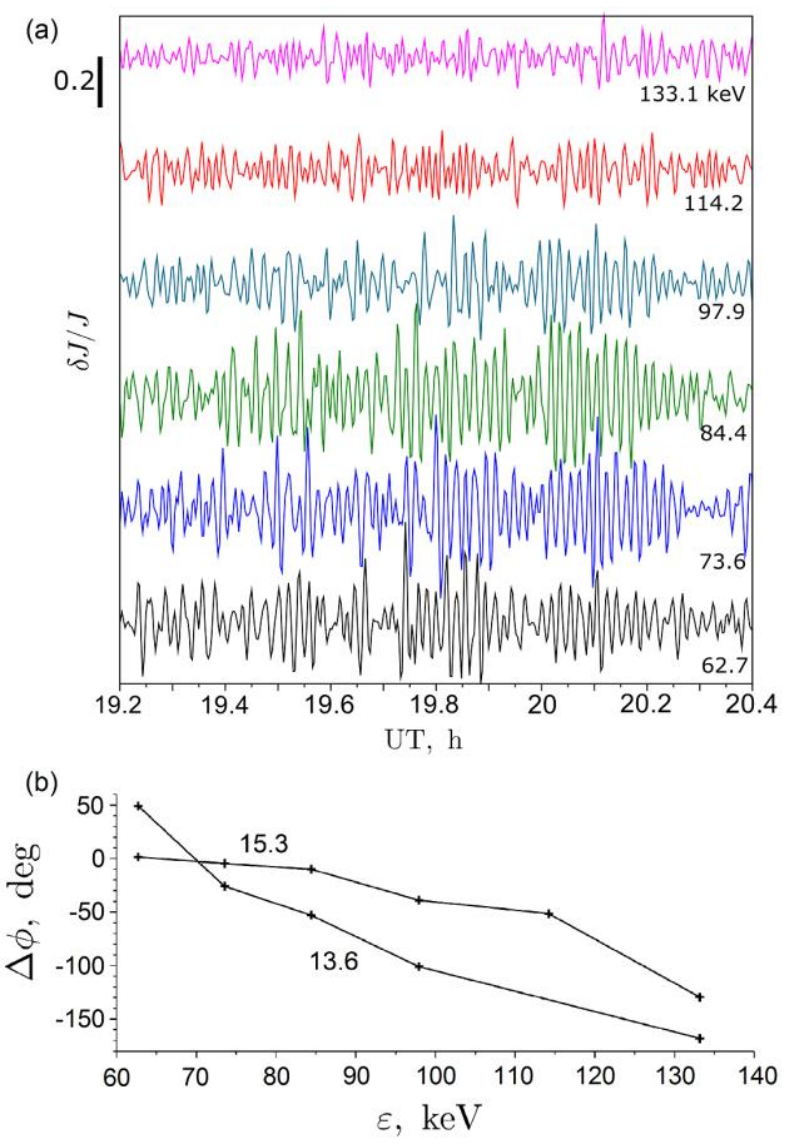

Figure 24. Oscillations of the proton flux $\delta J$ for different energies at a drift resonance with an Alfvén wave and the corresponding phase difference $\Delta \phi$ between $\delta J$ and the wave electric field azimuthal component $E_{\mathrm{a}}$ for the event described in [Mager et al., 2018]. For this event, the wave was a superposition of harmonics close in frequency (13.6 and 15.3 $\mathrm{MHz}$ ) of the transverse resonator for Alfvén waves (see Section 3.4)

\subsection{Drift-bounce resonance}

For the drift-bounce resonance at $K= \pm 1$ with the second harmonic, modulations of the particle flux or the distribution function near the geomagnetic equator $(l=0)$ are determined by expression [Chen and Hasegawa, 1994]

$$
\begin{aligned}
& \frac{\delta J}{J}=\frac{\delta F}{F}=i K \hat{\sigma} \frac{\overline{\delta S\left|\sin I_{0}^{l}\right|}}{\omega-m \bar{\omega}_{\mathrm{d}}-K \omega_{\mathrm{b}}} \times \\
& \times \frac{\hat{Q} F_{0}}{F_{0}} \exp \left(-\vec{k}_{\perp} \vec{\rho}\right),
\end{aligned}
$$

where the bar denotes averaging over the bounce period in the form

$$
\overline{(\ldots)}=\frac{4}{\tau_{\mathrm{b}}} \int_{0}^{l_{2}}(\ldots) \frac{d l}{\left|v_{\|}\right|} .
$$

As for the drift resonance, it follows from expression (126) that the maximum amplitude of the particle flux $\delta J$ oscillations is achieved at resonance, that is, at a given resonance pair of energy $\varepsilon_{\text {res }}$ and pitch angle $\alpha_{\text {res }}$. Besides, the phase of $\delta F$ oscillations changes by $180^{\circ}$ when passing through the resonant energy at a fixed pitch angle. The same happens when passing through the resonant pitch angle if we fix the particle energy. However, there are also significant differences from the drift resonance case. Since the sign of expression (126) depends on the sign of the parallel velocity of particles $\hat{\sigma}$, the particle flux oscillations with pitch angle $\alpha$ are in antiphase to the particle flux oscillations with a conjugate pitch angle $180^{\circ}-\alpha$. In addition, there are no particle flux oscillations with a pitch angle $\alpha=90^{\circ}$ since the second harmonic $\Phi$ and $B_{\|}$are equal to zero at the equator.

Let us find the phase relations between the particle flux oscillations and the wave magnetic field oscillations, namely, the radial component $B_{\mathrm{r}}$. It is more convenient to use a magnetic rather than electric field here since at the equator the electric field of the second harmonic is zero and changes sign, whereas the magnetic field has a maximum amplitude. As in the drift resonance case, we assume that the wave parallel magnetic field is absent, the particles are protons, $\omega_{\mathrm{d}}<0$, $m<0, \gamma>0, \hat{Q}>0$. Since $B_{\mathrm{r}} \propto m \partial \Phi / \partial l$,

$$
\delta J \propto-i K \hat{\sigma} \frac{B_{r}}{\omega-m \bar{\omega}_{\mathrm{d}}-K \omega_{\mathrm{d}}} \exp \left(-\vec{k}_{\perp} \vec{\rho}\right) .
$$

Thus, the phase difference $\Delta \phi$ between $B_{\mathrm{r}}$ and $\delta J$ at a fixed pitch angle $\alpha$ changes as follows depending on the particle energy

$$
\Delta \phi= \begin{cases}(K \hat{\sigma}+2) \pi / 2+\vec{k}_{\perp} \vec{\rho} & \varepsilon>\varepsilon_{\mathrm{res}} \\ (K \hat{\sigma}+1) \pi / 2+\vec{k}_{\perp} \vec{\rho} & \varepsilon=\varepsilon_{\mathrm{res}} . \\ K \hat{\sigma} \pi / 2+\vec{k}_{\perp} \vec{\rho} & \varepsilon<\varepsilon_{\mathrm{res}}\end{cases}
$$

It can be seen that if we do not take into account $\vec{k}_{\perp} \vec{\rho}$, at resonance energy $\varepsilon_{\text {res }}$ the particle flux oscillations are in phase or antiphase with the wave magnetic field 
radial component $B_{\mathrm{r}}$, depending on the type of driftbounce resonance $(K= \pm 1)$ and the direction of the parallel particle velocity ( $\hat{\sigma}=1$ for particles with $\alpha<90^{\circ}$ and $\hat{\sigma}=-1$ for particles with $\alpha>90^{\circ}$ ). Examples of the proton flux modulation at the drift-bounce resonance with a wave can be found in [Liu et al., 2013; Le et al., 2017; Min et al., 2017; Takahashi et al., 1990; Takahashi et al., 2018b; Wang et al., 2021]. The articles [Yang et al., 2011; Ren et al., 2016] provide examples of oxygen ion flux modulation. Pokhotelov et al. [2000] showed that the particle fluxes modulated in the driftbounce resonance can have an inverse effect on the same waves. It is the way how these authors explain the amplitude modulation of giant pulsations $(\mathrm{Pg})$.

\subsection{Finite gyroradius effect and determination of the wave vector transverse component}

The resonant perturbation by the wave of the particle distribution function (see (114)), and, consequently, of the flux, depends on the angle between the wave propagation direction, given by the transverse wave vector $\vec{k}_{\perp}$, and the direction of the radius vector $\vec{\rho}$ (gyroradius) drawn from the center of its rotation to the point where the particle is located. The gyroradius is usually small compared to the transverse wavelength. Nevertheless, the finite gyroradius effect can be used in practice to determine the transverse wave vector components $\vec{k}_{\perp}$, that is, to find the length and the propagation direction of a wave resonantly interacting with particles.

Let a satellite register particle fluxes with the same energies and pitch angles, i.e. with gyroradii equal in magnitude, but different in direction. Denote them by $\vec{\rho}_{1}$ and $\vec{\rho}_{2}$ (Figure 25), and $\left|\vec{\rho}_{1}\right|=\left|\vec{\rho}_{2}\right|=\rho$. This means that the satellite's detector registers particles whose gyration centers do not coincide but are at the same distance from the satellite in the plane transverse to the magnetic field (see Figure 25). As follows from Equation (114), the phase difference between oscillations of particle fluxes with different gyroradius directions is

$$
\triangle \phi_{12}=\phi_{1}-\phi_{2}=\vec{k}_{\perp} \vec{\rho}_{2}-\vec{k}_{\perp} \vec{\rho}_{1} .
$$

For example, if two particle fluxes with equal energies and pitch angles are known, but rotation centers of one particle flux (1) are located to the east of the satellite, and those of other flux (2) are to the west (see Figure 25),

$$
\triangle \phi_{12}=2 k_{\mathrm{a}} \rho \text {, }
$$

where $k_{\mathrm{a}}$ is the wave vector azimuthal component. If the measurements were carried out close to the geomagnetic equator, where $k_{\mathrm{a}}=m / L$, we can estimate the azimuthal wave number

$$
m=\frac{\Delta \phi_{12} L}{2 \rho} \text {. }
$$

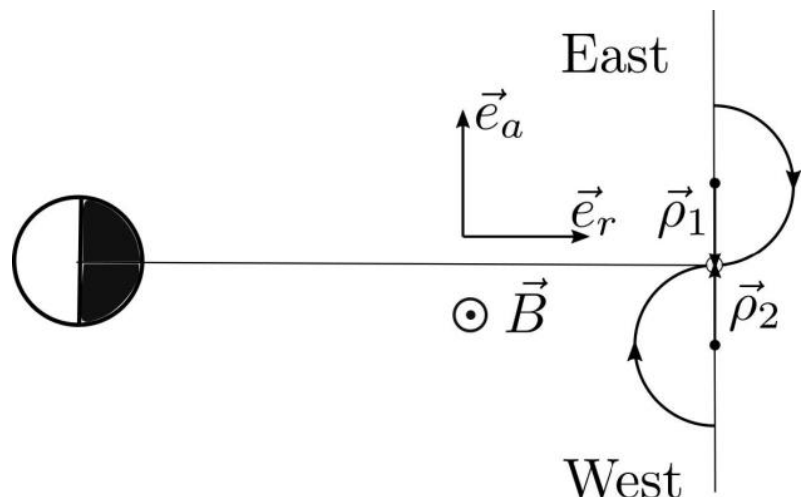

Figure 25. Diagram illustrating registration of protons by a satellite detector. Here, $\vec{e}_{\mathrm{a}}$ and $\vec{e}_{\mathrm{r}}$ are unit vectors in the direction of azimuthal and radial coordinates respectively

In the same way, it is possible to determine the wave vector radial component $k_{\mathrm{r}}$ if we find the phase difference of the particle fluxes with rotation centers located on the Earth-satellite line on the opposite sides relative to the satellite.

Examples of using this method for calculating the azimuthal wave number can be found in [Su et al., 1977; Lin et al., 1988; Min et al., 2017; Takahashi et al., 2018b].

\section{CONCLUSION}

Studies of the interaction between long-period ULF waves and particles date back to the mid-1960s [Dungey, 1964; Falthammar, 1965] and continued extensively in subsequent years. The important milestones in this field were the introduction of the concept of particle diffusion in a stochastic field of ULF waves [Falthammar, 1968; Brizard and Chan, 2001; Shprits et al., 2008]; revealing the role of these waves in the acceleration of ring current and radiation belt particles [Baker et al., 1987; Rostoker et al., 1998]; the elaboration of an idea of wave generation by plasma instabilities, caused by resonant energy transfer from high-energy particles [Mikhailovskii and Fridman, 1967; Hasegawa, 1969; Southwood et al., 1969; Mikhailovskii and Pokhotelov, 1975, 1976; Southwood, 1976; Hughes et al., 1978].

Both theory and experiment advanced significantly in the last decade. A remarkable progress was made in the theory of the ULF wave generation by plasma instabilities [Mager et al., 2013; Mager and Klimushkin, 2017; Yamakawa et al., 2020]. Evidence for the generation of azimuthally small-scale Alfvén waves by such instabilities was obtained from spacecraft observations [Dai et al., 2013; Liu et al., 2013; Min et al., 2017; Mager et al., 2018; Takahashi et al., 2018a, b; Oimatsu et al., 2018; Yamamoto et al., 2019; Wei et al., 2019]. The existence of drift compressional waves in the magnetosphere was confirmed [Chelpanov et al., 2016, 2018; Rubtsov et al., 2018; Mager et al., 2019]. The ability of ULF waves to efficiently accelerate particles in the ring current and radiation belts was demonstrated [Zong et al., 2009, 2012, 2017; Mann et al., 2013; Claudepierre et al., 2013; Foster et al., 2015; Hao et al., 2014, 2019; Ren et al., 2017; Ren et al., 
2019; Simms et al., 2021]. Understanding the structure of the radiation belts caused by diffusion across magnetic shells related to wave-particle resonance underwent significant advances [Ozeke et al., 2012, 2018; Mann et al., 2012; Su et al., 2015; Lejosne and Kollmann, 2020]. Methods of simulation of particle motion in the electromagnetic field of ULF waves were improved [Gubar', 2010; Degeling et al., 2019; Rankin et al., 2020].

In conclusion of this review, we note several unresolved issues in the theory of interaction of ULF waves and particles in the magnetosphere.

1. What is the role of the compressional component of the magnetic field and the parallel electric field of the wave in the energy exchange between the Alfvén wave and particles? It is currently assumed that in Equation (18) only the second term, the azimuthal component of the electric field, $E_{\mathrm{a}}$, is responsible for this exchange. The compressional component of the magnetic field $B_{\|}$ and the parallel electric field $E_{\|}$are considered negligible. This concept originates from one-fluid magnetohydrodynamics, where a parallel electric field is assumed to vanish identically, and the magnetic field of the Alfvén wave does not have the compressional component (unlike magnetic sound). However, as was discussed in Subsection 3.2, the Alfvén wave has a noticeable compressional component $B_{\|}$in an inhomogeneous plasma with curved field lines. Moreover, accounting for the kinetic effects and the field line curvature the Alfvén wave reveals a significant parallel electric field $E_{\|}$[Kostarev et al., 2021].

These fields can also contribute to energetic particle acceleration. However, these factors were not systematically taken into account.

2. The mechanisms of particle acceleration in radiation belts can be either resonant or nonresonant, and the resonant acceleration can be either difiusional or nondifiusional. The relative contribution of these mechanisms is unclear. The possibility of particle acceleration by azimuthally small-scale waves deserves special attention because these waves have a significant azimuthal component of the electric field and often feature a compressional part. Nonetheless, these waves do not meet the drift resonance condition. Thus, comprehensive studies of nonresonant mechanisms of particle acceleration by azimuthally small-scale waves are required. In addition, it is necessary to develop a more detailed theory of particle acceleration by nonstationary ULF waves with the finite extension in the azimuthal direction. Their precise frequency $w$ and azimuthal wave number $m$ are not defined in a strict sense. Therefore, in this case the distinction between resonant and nonresonant mechanisms blurs.

3. To date, the relative role of ULF and VLF waves (whistlers, chorus) in the acceleration of electrons in radiation belts remains unclear. There is both theoretical and experimental data on each of these mechanisms. At the same time, evidence is accumulated that the acceleration of electrons to relativistic energies requires the joint action of both mechanisms [O'Brien et al., 2003; Li et al., 2005; Simms et al., 2021]. For example,
Pilipenko et al. [2017] considered a two-stage process: first, electrons are accelerated by ULF waves and (due to diffusion) are transferred to the inner regions of the magnetosphere, where they undergo the action of VLF waves, which finally accelerate them to relativistic energies. Simms et al. [2021] have presented arguments in favor of the reverse sequence: first, particles are accelerated by VLF chorus, then the ULF waves carry them deep into the magnetosphere, accelerating about the required energies; at the same time, ULF waves have a stronger effect on the electron flux increase than UHF waves. Finally, in the magnetic pumping mechanism [Liu et al., 1999], VLF waves lead to pitchangular diffusion of particles, due to which the drift velocity of particles constantly changes and conditions for continuous energy transfer to particles from ULF waves are fulfilled. All these mechanisms, however, have so far been considered mainly at the qualitative level and need deeper theoretical study.

4. The theory of the wave generation by interaction with high-energy particles is developed for the case of stationary energetic particle distribution, which does not depend on the azimuthal coordinate. However, the real magnetosphere does not meet these conditions, first because the particle motion can be influenced by the electric convection field, which is axially asymmetric. Second, energetic particles can cluster in substorminjected clouds and move in the azimuthal direction, generating waves in accordance with the moving source theory (see Subsection 4.5). Aspects of wave generation under these conditions are far from clear.

5. The common identification of wave origin as external or internal may not fully be consistent with the available data. Hao et al. [2014]; Zong et al. [2017]; Hao et al. [2019] show that an impulse impact on the magnetosphere can lead to a generation of poloidal waves with $m>>1$, which subsequently participate in radiation belt electron acceleration. To explain this phenomenon, a mechanism for the generation of poloidal Alfvén waves at the plasmapause has been proposed [Zong et al., 2018]. However, the attribution of all these events to the plasmapause is questionable. Global Pc5 pulsations featuring practically the same amplitudes across large sections of Earth's surface during remarkably long time intervals (up to tens of hours) also worth mentioning here [Potapov et al., 2006]. Such waves exhibit a noticeable correlation with the solar wind velocity, which hints at their external sources. Nonetheless, there is data on their moderate to high azimuthal wave numbers [Potapov et al., 2011], widely thought as an indication of internal sources. In addition, global Pc5 pulsations play a significant role in magnetic storm development, which indicates their role in charged particle acceleration. On the other hand, statistics reveal that low-m toroidal waves are observed mainly in the auroral oval region [Kozyreva et al., 2016], although the generation of such waves and the formation of the oval seem to be unrelated. Could energetic particles be responsible for the generation of some low- $m$ toroidal waves at the auroral oval region?

6. The theory of drift compression waves in Earth's magnetosphere is far from complete. In particular, we 
do not know their structure across magnetic shells, the role of space plasma external currents in their generation, aspects that influence the coupling of these waves with the Alfvén mode. Without answering these questions, reliable identification of drift compression waves in the magnetosphere is impossible.

7. The role of pressure anisotropy in the magnetospheric wave generation seems to be poorly studied. Anisotropy is generally believed to be responsible for the drift-mirror mode generation. However, the theory of drift-mirror waves is developed only for longitudinally homogeneous plasma. As discussed in Subsection 4.5, the presence of a parallel magnetic field inhomogeneity can modify conditions for the mirror instability. At the same time, the pressure anisotropy is a natural result of the magnetospheric plasma convection. Taking it into account this factor seems to be fundamentally important for understanding the generation of magnetospheric waves due to transfer of energy from particles to waves.

The reported study was funded by RFBR, project number 20-15-50241. The authors are indebted to O.V. Mager and A.V. Rubtsov for discussion and comments.

\section{REFERENCES}

Allan W., Poulter E. M., Nielsen E. STARE observations of a Pc5 pulsation with large azimuthal wave number. J. Geophys. Res. 1982, vol. 87, pp. 6163-6172. DOI: 10.1029/ JA087iA08p06163.

Allison H.J., Shprits Y.Y. Local heating of radiation belt electrons to ultra-relativistic energies. Nature Communications. 2020, vol. 11, article id. 4533. DOI: 10.1038/s41467-020-18053-z.

Alperovich L.S., Fedorov E.N. Hydromagnetic Waves in the Magnetosphere and the Ionosphere. Springer, 2007. 426 p. DOI: 10.1007/978-1-4020-6637-5.

Anderson B. J. Statistical studies of Pc3-5 pulsations and their relevance for possible source mechanisms of ULF waves. Ann. Geophys. 1993, vol. 11, pp. 128-143.

Antonsen T.M., Jr. and. Lane B. Kinetic equations for low frequency instabilities in inhomogeneous plasmas. Phys. Fluid. 1980, vol. 23, pp. 1205-1214. DOI: 10.1063/1.863121.

Baddeley L.J., Yeoman T.K., Wright D.M., Davies J.A., Trattner K. J., and Roeder J. L. Morning sector drift-bounce resonance driven ULF waves observed in artificially-induced HF radar backscatter. Ann. Geophys. 2002, vol. 20, pp. 14871498. DOI: 10.5194/angeo-20-1487-2002.

Baddeley L.J., Yeoman T.K., Wright D.M., Trattner K.J., Kellet B.J. Statistical study of unstable particle populations in the global ring current and their relation to the generation of high m ULF waves. Ann. Geophys. 2004, vol. 22, pp. 42294241. DOI: 10.5194/angeo-22-4229-2004.

Baddeley L.J., Yeoman T.K., Wright D.M., Trattner K.J., Kellet B.J. On the coupling between unstable magnetospheric particle populations and resonant high $\mathrm{m}$ ULF wave signatures in the ionosphere. Ann. Geophys. 2005, vol. 23, pp. 567-577. DOI: 10.5194/angeo-23-567-2005.

Baddeley L.J., Lorentzen D.A., Partamies N., Denig M., Pilipenko V.A., Oksavik K., Chen X., Zhang Y. Equatorward propagating auroral arcs driven by ULF wave activity: Multipoint ground- and space-based observations in the dusk sector auroral oval. J. Geophys. Res.: Space Phys. 2017, vol. 122 (5), pp. 5591-5605. DOI: 10.1002/2016JA023427.

Baker D.N. Satellite Anomalies due to Space Storms. Space Storms and Space Weather Hazards, pp. 251-284, New
York, Springer, 2001. DOI: 10.1007/978-94-010-0983-6_11.

Baker D.N., Belian R.D., Higbie P.R., Klebesadel R.W., Blake J.B. Deep dielectric charging effects due to high-energy electrons in earth's outer magnetosphere. J. Electrostatics. 1987, vol. 20(1), pp. 3-19. DOI: 10.1016/03043886(87)90082-9.

Baker D.N., Pulkkinen T.I., Li X., Kanekal S.G., Ogilvie K.W., Lepping R.P., Blake J.B., Callis L.B., Rostoker G., Singer H.J., Reeves G.D. A strong CME-related magnetic cloud interaction with the Earth's magnetosphere: ISTP observations of rapid relativistic electron acceleration on May 15, 1997. Geophys. Res. Lett. 1998, vol. 25 (15), pp. 29752978. DOI: $10.1029 / 98$ GL01134.

Baker D.N., Hoxie V., Zhao H., Jaynes A.N., Kanekal S., Li X., Elkington S. Multiyear measurements of radiation belt electrons: acceleration, transport, and loss. J. Geophys. Res.: Space Phys. 2019. Vol. 124, no. 4. P. 2588-2602. DOI: 10.1029/2018JA026259.

Barfield J.N., Pherron R.L. Statistical characteristics of storm-associated Pc 5 micropulsations observed at the synchronous equatorial orbit. J. Geophys. Res. 1972, vol. 77, pp. 4720-4733. DOI: 10.1029/JA077i025p04720.

Beharrell M., Kavanagh A.J., Honary F. On the origin of high- $m$ magnetospheric waves. J. Geophys. Res.: Space Phys. 2010, 115 (A2), p. A02201. DOI: 10.1029/2009JA014709.

Brizard A.J., Chan A.A. Relativistic bounce-averaged quasilinear diffusion equation for low-frequency electromagnetic fluctuations. Physics of Plasmas. 2001, vol. 8, pp. 4762-4771. DOI: 10.1063/1.1408623.

Catto P.J., Tang W.M., Baldwin D.E. Generalized gyrokinetics. Plasma Physics. 1981, vol. 23, pp. 639-650. DOI: 10.1088/0032-1028/23/7/005.

Chelpanov M.A., Mager P.N., Klimushkin D.Yu., Berngardt O.I., Mager O.V. Experimental evidence of drift compressional waves in the magnetosphere: An Ekaterinburg coherent decameter radar case study. J. Geophys. Res.: Space Phys. 2016, vol. 121, pp. 1315-1326. DOI: 10.1002/ 2015JA022155.

Chelpanov M.A., Mager O.V., Mager P.N., Klimushkin D.Yu., and Berngardt O.I. Properties of frequency distribution of Pc5-range pulsations observed with the Ekaterinburg decameter radar in the nightside ionosphere. J. Atmos. SolarTerr. Phys. 2018, vol. 167, pp. 177-183. DOI: 10.1016/ j.jastp.2017.12.002.

Chelpanov M.A., Mager P.N., Klimushkin D.Yu., Mager O.V. Observing magnetospheric waves propagating in the direction of electron drift with Ekaterinburg Decameter Coherent Radar. Solar-Terr. Phys. 2019, vol. 5, pp. 51-57. DOI: $10.12737 /$ stp-51201907.

Chen L., Cowley S.C. On field line resonances of hydromagnetic Alfvén waves in dipole magnetic field. Geophys. Res. Lett. 1989, vol. 16, pp. 895-897. DOI: 10.1029/GL016i008p00895.

Chen L., Hasegawa A. A theory of long period magnetic pulsation. 1: Steady state excitation of a field line resonance. J. Geophys. Res. 1974, vol. 79, pp. 1024-1032. DOI: 10.1029/JA079i007p01024.

Chen L., Hasegawa A. Kinetic theory of geomagnetic pulsations: 1. Internal excitations by energetic particles. $J$. Geophys. Res. 1991, vol. 96, pp. 1503-1512. DOI: 10.1029/90JA02346.

Chen L., Hasegawa A. Kinetic theory of geomagnetic pulsations: 2. Ion flux modulations by transverse waves. $J$. Geophys. Res. 1994, vol. 99, pp. 179-182. DOI: 10.1029/93JA02774.

Cheng C.Z., Lin C.S. Eigenmode analysis of compressional waves in the magnetosphere. Geophys. Res. Lett. 1987, vol. 14 (8), pp. 884-887. DOI: 10.1029/GL014i008p00884.

Cheng C.Z., Qian Q. Theory of ballooning-mirror 
instabilities for anisotropic pressure plasmas in the magnetosphere. J. Geophys. Res. 1994, vol. 99 (A6), pp. 11193-11209. DOI: 10.1029/94JA00657.

Cheremnykh O.K., Parnowski A.S. The theory of ballooning perturbations in the inner magnetosphere of the Earth. Adv. Space Res. 2004, vol. 33, pp. 769-773. DOI 10.1016/S0273-1177(03)00642-2.

Chisham G., Orr D., Yeoman T.K. Observations of a giant pulsation across an extended array of ground magnetometers and on auroral radar. Planet. Space Sci. 1992, vol. 40, pp. 953-964. DOI: 10.1016/0032-0633(92)90135-B.

Choi J., Lee D.-H. On the persistent poloidal Alfvén waves. Geophys. Res. Lett. 2021, vol. 48 (12), pp. e2021GL092945. DOI: 10.1029/2021GL092945.

Claudepierre S.G., Mann I.R., Takahashi K., Fennell J.F., Hudson M.K., Blake J.B., et al. Van Allen Probes observation of localized drift resonance between poloidal mode ultra-low frequency waves and $60 \mathrm{keV}$ electrons. Geophys. Res. Lett. 2013, vol. 40, pp. 4491-4497. DOI: 10.1002/grl.50901.

Cooper M.B., Gerrard A.J., Lanzerotti L.J., Soto-Chavez A.R., Kim H., Kuzichev I.V., and Goodwin L.V. Mirror instabilities in the inner magnetosphere and their potential for localized ULF wave generation. J. Geophys. Res.: Space Phys. 2021, vol. 126(2), p. e2020JA028773. DOI: 10.1029/2020JA028773.

Crabtree C., Chen L. Finite gyroradius theory of drift compressional modes. Geophys. Res. Lett. 2004, vol. 31, pp. L17804. DOI: 10.1029/2004GL020660.

Crabtree C., Horton W., Wong H.V., van Dam J.W. Bounce-averaged stability of compressional modes in geotail flux tubes. J. Geophys. Res. 2003, vol. 108, pp. 1084. DOI: 10.1029/2002JA009555.

Cummings W.D., O’Sullivan R.J., Coleman P.J. Standing Alfvén waves in the magnetosphere. J. Geophys. Res. 1969, vol. 74, pp. 778-793. DOI: 10.1029/JA074i003p00778.

Da Silva L.A., Shi J., Alves L.R., Sibeck D., Marchezi J.P., Medeiros C., et al. High-energy electron flux enhancement pattern in the outer radiation belt in response to the Alfvénic fluctuations within high-speed solar wind stream: A statistical analysis. J. Geophys. Res.: Space Phys. 2021, vol. 126, no. 8. DOI: 10.1029/2021JA029363.

Dai L., Takahashi K., Wygant J.R., Chen L., Bonnell J., Cattell C.A., et al. Excitation of poloidal standing Alfvén waves through drift resonance wave-particle interaction. Geophys. Res. Lett. 2013, vol. 40, pp. 4127-4132. DOI: 10.1002/grl.50800

Degeling A.W., Rankin R., Kabin K., Marchand R., Mann I.R. The effect of ULF compressional modes and field line resonances on relativistic electron dynamics. Planet. Space Sci. 2007, vol. 55(6), pp. 731-742. DOI 10.1016/j.pss.2006.04.039.

Degeling A.W., Ozeke L.G., Rankin R., Mann I.R., and Kabin K. Drift resonant generation of peaked relativistic electron distributions by Pc 5 ULF waves. J. Geophys. Res.: Space Phys. 2008, vol. 113 (A2), pp. A02208. DOI 10.1029/2007JA012411.

Degeling A.W., Rankin R., Wang Y., Shi Q.Q., Zong Q.G. Alteration of particle drift resonance dynamics near poloidal mode field line resonance structures. J. Geophys. Res.: Space Phys. 2019, vol. 124 (9), pp. 7385-7401. DOI: 10.1029/2019JA026946.

Degtyarev V.I., Kharchenko I.P., Potapov A.S., Tsegmed B., Chudnenko S.E. Qualitative estimation of magnetic storm efficiency in producing relativistic electron flux in the Earth's outer radiation belt using geomagnetic pulsations data. $A d v$. Space Res. 2009, vol. 43, pp. 829-836. DOI 10.1016/j.asr.2008.07.004.

Demekhov A.G. Recent progress in understanding Pc1 pearl formation. J. Atmos. Solar-Terr. Phys. 2007, vol. 69, pp.
1599-1774. DOI: 10.1016/j.jastp.2007.01.014.

Demekhov A.G., Trakhtengerz V.Yu., Rycroft M., Nann D. Electron acceleration in the magnetosphere by whistler-mode waves of varying frequency. Geomagnetism and Aeronomy. 2006, vol. 46, iss. 6, pp. 711-716. DOI: 10.1134/S0016793206060053.

Drozdov A.Y., Allison H.J., Shprits Y.Y., Elkington S.R., Aseev N.A. A comparison of radial diffusion coefficients in 1D and 3D long-term radiation belt simulations. J. Geophys. Res.: Space Phys. 2021. Vol. 126, no. 8, article id. e28707. DOI: $10.1029 / 2020 J A 028707$.

Dungey J.W. Electrodynamics of the Outer Atmospheres. Pennsylvania State University, Ionosphere Research Laboratory, 1954. 52 p.

Dungey J.W. Effects of electromagnetic perturbations on particles trapped in the radiation belts. Space Sci. Rev. 1964, vol. 4, pp. 199-222. DOI: 10.1007/BF00173882.

Elkington S.R. A Review of ULF Interactions with Radiation Belt Electrons. Magnetospheric ULF Waves: Synthesis and New Directions. Geophys. Monograph Ser. 2006, vol. 169, pp. 177-194, Washington: American Geophysical Union Publ., DC, USA, 2006. DOI: 10.1029/169GM12.

Elkington S.R., Sarris T.E. The role of Pc-5 ULF waves in the radiation belts: Current understanding and open questions. Waves, Particles, and Storms in Geospace. 2016, pp. 80-101. Oxford University Press, 2016. DOI: 10.1093/acprof:oso/ 9780198705246.003.0005.

Elkington S.R., Hudson M.K., Chan A.A. Acceleration of relativistic electrons via drift-resonant interaction with toroidal-mode Pc-5 ULF oscillations. Geophys. Res. Lett. 1999, vol. 26 (21), pp. 3273-3276. DOI: 10.1029/ 1999GL003659.

Elkington S.R., Hudson M.K., Chan A.A. Resonant acceleration and diffusion of outer zone electrons in an asymmetric geomagnetic field. J. Geophys. Res. 2003, vol. 108 (A3), pp. 1116. DOI: 10.1029/2001JA009202.

Fälthammar C.-G. Effects of time-dependent electric fields on geomagnetically trapped radiation. J. Geophys. Res. 1965, vol. 70 (11), pp. 2503-2516. DOI: 10.1029/JZ070i011p02503.

Fälthammar C.-G. Radial diffusion by violation of the third adiabatic invariant. Earth's Particles and Fields. 1968, pp. $157-169$.

Fei Y., Chan A.A., Elkington S.R., Wiltberger M.J. Radial diffusion and mhd particle simulations of relativistic electron transport by ULF waves in the September 1998 storm. $J$. Geophys. Res.: Space Phys. 2006, vol. 111 (A12), pp. A12209. DOI: 10.1029/2005JA011211.

Feygin F. Z., Khabazin Yu. G. Slow drift mirror kinetic instability at a finite electron temperature in a nonmaxwellian space plasma. Geomagnetism and Aeronomy. 2014, vol. 54 (6), pp. 727-734. DOI: 10.1134/S0016793214060103.

Foster J.C., Wygant J.R., Hudson M.K., Boyd A.J., Baker D.N., Erickson P.J., and Spence H.E. Shock induced prompt relativistic electron acceleration in the inner magnetosphere. $J$. Geophys. Res.: Space Phys. 2015, vol. 120 (3), pp. 16611674. DOI: $10.1002 / 2014 J A 020642$.

Glassmeier K.-H. Reply to the comment by I.R. Mann and G. Chisham. Ann. Geophys. 2000, vol. 18, pp. 167-169. DOI: 10.1007/s00585-000-0167-y.

Glassmeier K.-H., Buchert S., Motschmann U., Korth A., Pedersen A. Concerning the generation of geomagnetic giant pulsations by drift-bounce resonance ring current instabilities. Ann. Geophys. 1999, vol. 17, pp. 338-350. DOI: 10.1007/s00585-999-0338-4.

Gubar' Yu. I. Drift resonance of relativistic electrons with ULF waves as a nonlinear resonance. Cosmic Res. 2010, vol. 48 (4), pp. 300-307. DOI: 10.1134/S0010952510040039.

Guglielmi A. and Potapov A. Frequency-modulated ULF waves in near-Earth space. Phys. Usp. 2021, vol. 64(5), pp. 
87-92. DOI: 10.3367/UFNe.2020.06.038777.

Guglielmi A.V., Zolotukhina N.A. Excitation of Alfvén oscillations of the magnetosphere by the asymmetric ring current. Issledovaniya po geomagnetizmu, aeronomii i fizike Solntsa [Research on Geomagnetism, Aeronomy and Solar Physics]. Nauka Publ., 1980, iss. 50, pp. 129-137. (In Russian),

Hamlin D.A., Karplus R., Vik R.C., Watson K.M. Mirror and azimuthal drift frequencies for geomagnetically trapped particles. J. Geophys. Res. 1961, vol. 66 (1) , pp. 1-4. DOI: 10.1029/JZ066i001p00001.

Hao Y.X., Zong Q.-G., Wang Y.F., Zhou X.-Z., Zhang H., Fu S.Y., et al. Interactions of energetic electrons with ULF waves triggered by interplanetary shock: Van Allen Probes observations in the magnetotail. J. Geophys. Res.: Space Phys. 2014, vol. 119, pp. 8262-8273. DOI: 10.1002/2014JA020023.

Hao Y.X., Zong Q.-G., Zhou X.-Z., Rankin R., Chen X.R., Liu Y., et al. Global-scale ULF waves associated with SSC accelerate magnetospheric ultrarelativistic electrons. $J$. Geophys. Res.: Space Phys. 2019, vol. 124 (3), pp. 15251538. DOI: 10.1029/2018JA026134.

Hasegawa A. Drift mirror instability of the magnetosphere. Phys. Fluids. 1969, vol. 12, pp. 2642-2650. DOI: $10.1063 / 1.1692407$.

Higuchi T., Kokubun S. Waveform and polarization of compressional $\mathrm{Pc}-5$ waves at geosynchronous orbit. $J$. Geophys. Res. 1988, vol. 93, pp. 14433-14443. DOI 10.1029/JA093iA12p14433.

Huba J.D., Drake J.F. Physical mechanism of waveparticle resonances in an inhomogeneous magnetic field. I - Linear theory. Phys. Fluids. 1981, vol. 24, pp.1650-1654 DOI: $10.1063 / 1.863588$

Huba J.D., Drake J.F. Physical mechanism of waveparticle resonances in a curved magnetic field. Phys. Fluids. 1982, vol. 25, pp. 1207-1210. DOI: 10.1063/1.863891.

Hudson M.K., Elkington S.R., Lyon J.G., Goodrich C.C., Rosenberg T.J. Simulation of Radiation Belt Dynamics Driven by Solar Wind Variations. Sun-Earth Plasma Connections. Geophys. Monograph Ser. 1999, vol. 109, pp. 171-182. Washington: American Geophysical Union Publ., DC, USA, 1999. DOI: $10.1029 / \mathrm{GM} 109$ p0171.

Hughes W.J., Southwood D.J., Mauk B., McPherron R.L., Barfield J.N. Alfvén waves generated by an inverted plasma energy distribution. Nature. 1978, vol. 275, pp. 43-45. DOI: 10.1038/275043a0.

Hughes W.J., McPherron R.L., Barfield J.N., Mauk B.H. A compressional Pc4 pulsation observed by three satellites in geostationary orbit near local midnight. Planet. Space Sci. 1979, vol. 27, pp. 821-840. DOI: 10.1016/00320633(79)90010-2.

James M.K., Yeoman T.K., Mager P.N., Klimushkin D.Yu. The spatio-temporal characteristics of ULF waves driven by substorm injected particles. J. Geophys. Res.: Space Phys. 2013, vol. 118, pp. 1737-1749. DOI: 10.1002/jgra.50131.

Karpman V.I., Meerson B.I., Mikhailovsky A.B., Pokhotelov O.A. The effects of bounce resonances on wave growth rates in the magnetosphere. Planet. Space Sci. 1977 , vol. 25, pp. 573-585. DOI: 10.1016/0032-0633(77)90064-2.

Klimushkin D.Yu. Method of description of the Alfvén and magnetosonic branches of inhomogeneous plasma oscillations. Plasma Phys. Rep. 1994, vol. 20, pp. 280-286.

Klimushkin D.Yu. Resonators for hydromagnetic waves in the magnetosphere. J. Geophys. Res. 1998, vol. 103, pp. 23692375. DOI: 10.1029/97JA02193.

Klimushkin D.Yu. The propagation of high-m Alfvén waves in the Earth's magnetosphere and their interaction with high-energy particles. J. Geophys. Res. 2000, vol. 105, pp. 23,303-23,310. DOI: 10.1029/1999JA000396.
Klimushkin D.Yu., Chen L. Eigenmode stability analysis of drift-mirror modes in nonuniform plasmas. Ann. Geophys. 2006, vol. 24 (10) , pp. 2435-2439. DOI: 10.5194/angeo-242435-2006.

Klimushkin D.Yu., Kostarev D.V. Two kinds of mirror modes in a nonzero electron-temperature plasma. Plasma Physics and Controlled Fusion. 2012, vol. 54 (9), pp. 092001. DOI: 10.1088/0741-3335/54/9/092001.

Klimushkin D.Yu., Mager P.N. The spatio-temporal structure of impulse-generated azimuthal small-scale Alfvén waves interacting with high-energy charged particles in the magnetosphere. Ann. Geophys. 2004, vol. 22, pp. 1053-1060. DOI: 10.5194/angeo-22-1053-2004.

Klimushkin D.Yu., Mager P.N. Spatial structure and stability of coupled Alfvén and drift compressional modes in non-uniform magnetosphere: Gyrokinetic treatment. Planet. Space Sci. 2011, vol. 59, pp. 1613-1620. DOI: 10.1016/j.pss.2011.07.010.

Klimushkin D.Yu., Mager P.N. Coupled Alfvén and driftmirror modes in non-uniform space plasmas: a gyrokinetic treatment. Plasma Physics and Controlled Fusion. 2012, vol. 54 (1), pp. 015006. DOI: 10.1088/0741-3335/54/1/015006.

Klimushkin D.Yu., Leonovich A.S., and Mazur V.A. On the propagation of transversally small-scale standing Alfvén waves in a three-dimensionally inhomogeneous magnetosphere. J. Geophys. Res. 1995, vol. 100, pp. $9527-$ 9534. DOI: 10.1029/94JA03233.

Klimushkin D.Yu., Mager P.N., Glassmeier K.-H. Toroidal and poloidal Alfvén waves with arbitrary azimuthal wave numbers in a finite pressure plasma in the Earth's magnetosphere. Ann. Geophys. 2004, vol. 22, pp. 267-288. DOI: 10.5194/angeo-22-267-2004.

Klimushkin D.Yu., Mager P.N., Pilipenko V.A. On the ballooning instability of the coupled Alfvén and drift compressional modes. Earth, Planets and Space. 2012, vol. 64, pp. 777-781. DOI: 10.5047/eps.2012.04.002.

Korablev L.V., Rudakov L.I. Instability of a plasma with an isotropic distribution function. Sov. Phys. JETP, Engl. Transl. 1968, vol. 27, pp. 439-440.

Kostarev D.V., Mager P.N. Drift-compression waves propagating in the direction of energetic electron drift in the magnetosphere. Solar-Terr. Phys. 2017, vol. 3, pp. 18-27. DOI: $10.12737 /$ stp-33201703.

Kostarev D.V., Mager P.N., Klimushkin D.Yu. Alfvén wave parallel electric field in the dipole model of the magnetosphere: gyrokinetic treatment. J. Geophys. Res.: Space Phys. 2021, vol. 126 (2), pp. e2020JA028611. DOI: 10.1029/2020JA028611.

Kozyreva O., Pilipenko V., Engebretson M.J., Yumoto K., Watermann J., Romanova N. In search of a new ULF wave index: Comparison of Pc5 power with dynamics of geostationary relativistic electrons. Planet. Space Sci. 2007, vol. 55 (6), pp. 755-769. DOI: 10.1016/j.pss.2006.03.013.

Kozyreva O.V., Pilipenko V.A., Engebretson M.J. Klimushkin D.Yu., Mager P.N Solar-Terr. Phys. 2016, vol. 2, pp. 35-45. DOI: $12737 / 16848$.

Lanzerotti L.J., Hasegawa A., Maclennan C.G. Drift mirror instability in the magnetosphere: Particle and field oscillations and electron heating. J. Geophys. Res. 1969, vol. 74 (24) , pp. 5565-5578. DOI: 10.1029/JA074i024p05565.

Le G., Chi P.J., Strangeway R.J., Russell C.T., Slavin J.A., Takahashi K., Singer H.J., et al. Global observations of magnetospheric high- $m$ poloidal waves during the 22 June 2015 magnetic storm. Geophys. Res. Lett. 2017, vol. 44, pp. 3456-3464. DOI: 10.1002/2017GL073048.

Lejosne S. Analytic expressions for radial diffusion. $J$. Geophys. Res.: Space Phys. 2019, vol. 124(6), pp. 4278-4294. DOI: 10.1029/2019JA026786. 
Lejosne S., Kollmann P. Radiation belt radial diffusion at Earth and beyond. Space Sci. Rev. 2020, vol. 216 (1), p. 19 DOI: $10.1007 / \mathrm{s} 11214-020-0642-6$.

Leonovich A.S., Mazur V.A. A theory of transverse smallscale standing Alfvén waves in an axially symmetric magnetosphere. Planet. Space Sci. 1993, 41, pp. 697-717. DOI: 10.1016/0032-0633(93)90055-7.

Leonovich A.S., Mazur V.A. Magnetospheric resonator for transverse-small-scale standing Alfvén waves. Planet. Space Sci. 1995, vol. 43, pp. 881-883, DOI: 10.1016/00320633(94)00206-7.

Leonovich A.S., Mazur V.A. Penetration to the Earth's surface of standing Alfvén waves excited by external currents in the ionosphere. Ann. Geophys. 1996, vol. 14, pp. 545-556. DOI: 10.1007/s00585-996-0545-1.

Leonovich A.S., Mazur V.A. Standing Alfvén waves in an axisymmetric magnetosphere excited by a non-stationary source. Ann. Geophys. 1998, vol. 16, pp. 914-920. DOI: 10.1007/s00585-998-0914-z.

Leonovich A.S., Mazur V.A. Lineynaya teoriya MGD kolebanii v magnitosfere [Linear theory of MHD oscillations in the magnetosphere]. Moscow, Fizmatlit, 2016. 480 p. (In Russian).

Leonovich A.S., Klimushkin D.Yu., Mager P.N. Experimental evidence for the existence of monochromatic transverse small-scale standing Alfvén waves with spatially dependent polarization. J. Geophys. Res.: Space Phys. 2015 , vol. 120, pp. 5443-5454. DOI: 10.1002/2015JA021044.

Li L., Cao J., and Zhou G. Combined acceleration of electrons by whistler-mode and compressional ULF turbulences near the geosynchronous orbit. J. Geophys. Res.: Space Phys. 2005, vol. 110 (A3), pp. A03203. DOI: 10.1029/2004JA010628.

Lifshits A.E., Fedorov E.N. Hydromagnetic oscillations of the magnetospheric-ionospheric resonator. Doklady AN SSSR [Reports of AS USSR]. 1986, vol. 287, pp. 90-94. (In Russian).

Lin C.S., Parks G.K. The coupling of Alfvén and compressional waves. J. Geophys. Res. 1978, vol. 83 (A6), pp. 2628-2636. DOI: 10.1029/JA083iA06p02628.

Lin N., McPherron R.L., Kivelson M.G., Williams D.J. An unambiguous determination of the propagation of a compressional Pc-5 wave. J. Geophys. Res. 1988, vol. 93 (A6), pp. 5601-5612. DOI: 10.1029/JA093iA06p05601.

Liu W.W., Rostoker G., Baker D. N. Internal acceleration of relativistic electrons by large amplitude ULF pulsations. $J$. Geophys. Res.: Space Phys. 1999, vol. 104 (A8), pp. 1739117407. DOI: 10.1029/1999JA900168.

Liu W., Cao J.B., Li X., Sarris T.E., Zong Q.-G., Hartinger M., et al. Poloidal ULF wave observed in the plasmasphere boundary layer. J. Geophys. Res.: Space Phys. 2013, vol. 118 (7), pp. 4298-4307. DOI: 10.1002/jgra.50427.

Longmire C. L. Elementary Plasma Physics. Interscience Publ. New York, London, 1963. 296 p.

Loto'aniu T.M., Mann I.R., Ozeke L.G., Chan A.A., Dent Z.C., Milling D.K. Radial diffusion of relativistic electrons into the radiation belt slot region during the 2003 Halloween geomagnetic storms. J. Geophys. Res.: Space Phys. 2006, vol 111 (A4), pp. A04218. DOI: 10.1029/2005JA011355.

Mager O.V. Alfvén waves generated through the driftbounce resonant instability in the ring current: A THEMIS multi-spacecraft case study. J. Geophys. Res.: Space Phys. 2021, vol. 126, iss. 11. e2021JA029241. DOI: 10.1029/2021 JA029241.

Mager O.V., Chelpanov M.A., Mager P.N., Klimushkin D.Yu., Berngardt O.I. Conjugate ionosphere-magnetosphere observations of a sub-Alfvénic compressional intermediate- $m$ wave: A case study using EKB Radar and Van Allen Probes. J. Geophys. Res.: Space Phys. 2019, vol. 124 (5), pp. 3276-
3290. DOI: $10.1029 / 2019 J A 026541$.

Mager P.N., Klimushkin D.Yu. Spatial localization and azimuthal wave numbers of Alfvén waves generated by driftbounce resonance in the magnetosphere. Ann. Geophys. 2005, vol. 23, pp. 3775-3784. DOI: 10.5194/angeo-23-3775-2005.

Mager P.N., Klimushkin D.Yu. Generation of Alfvén waves by a plasma inhomogeneity moving in the Earth's magnetosphere. Plasma Phys. Rep. 2007, vol. 33, pp. 391398. DOI: 10.1134/S1063780X07050042.

Mager P. N., Klimushkin D. Yu. Alfvén ship waves: high$\mathrm{m}$ ULF pulsations in the magnetosphere, generated by a moving plasma inhomogeneity. Ann. Geophys. 2008, vol. 26, pp. 1653-1663. DOI: 10.5194/angeo-26-1653-2008.

Mager P.N., Klimushkin D.Yu. Giant pulsations as modes of a transverse Alfvénic resonator on the plasmapause. Earth, Planets and Space. 2013, vol. 65, pp. 397-409. DOI: 10.5047/eps.2012.10.002.

Mager P.N., Klimushkin D.Yu. Non-resonant instability of coupled Alfvén and drift compressional modes in magnetospheric plasma. Plasma Physics and Controlled Fusion, vol. 59 (9) , pp. 095005, 2017. DOI: 10.1088/13616587/aa790c.

Mager P.N., Klimushkin D.Yu. The field line resonance in the three-dimensionally inhomogeneous magnetosphere: principal features. J. Geophys. Res.: Space Phys. 2021, vol. 126 (1) , p. e2020JA028455. DOI: 10.1029/2020JA028455.

Mager P.N., Klimushkin D.Yu., Kostarev D.V. Driftcompressional modes generated by inverted plasma distributions in the magnetosphere. J. Geophys. Res.: Space Phys. 2013, vol. 118, pp. 4915-4923. DOI: 10.1002/jgra.50471

Mager P.N., Berngardt O.I., Klimushkin D.Yu., Zolotukhina N.A., Mager O.V. First results of the high resolution multibeam ULF wave experiment at the Ekaterinburg SuperDARN Radar: Ionospheric signatures of coupled poloidal Alfvén and drift-compressional modes. $J$. Atmos. Solar-Terr. Phys. 2015, vol. 130-131, pp. 112-126. DOI: 10.1016/j.jastp.2015.05.017.

Mager P.N., Mikhailova O.S., Mager O.V., Klimushkin D.Yu. Eigenmodes of the Transverse Alfvénic resonator at the plasmapause: A Van Allen Probes case study. Geophys. Res. Lett. 2018, vol. 45, pp. 10,796-10,804. DOI: 10.1029/2018GL079596.

Mann I.R., Chisham G. Comment on "Concerning the generation of geomagnetic giant pulsations by drift-bounce resonance ring current instabilities" by K.-H. Glassmeier et al. Ann. Geophysicae, vol. 17, 338-350, (1999). Ann. Geophys. 2000, vol. 18, pp. 161-166. DOI: 10.1007/s00585-000-0161-4.

Mann I.R., Wright A.N. Finite lifetimes of ideal poloidal Alfvén waves. J. Geophys. Res. 1995, vol. 100, pp. $23677-$ 23686. DOI: $10.1029 / 95 J A 02689$.

Mann I.R., Murphy K.R., Ozeke L.G., Rae I.J., Milling D.K., Kale A.A., Honary F.F. The Role of Ultralow Frequency Waves in Radiation Belt Dynamics. Geophys. Monograph Ser. 2012, vol. 199, pp. 69-92. Washington: American Geophysical Union Publ., DC, USA, 2012. DOI: 10.1029/2012GM001349.

Mann I.R., Lee E.A., Claudepierre S.G., Fennell J.F., Degeling A., Rae I.J., et al. Discovery of the action of a geophysical synchrotron in the Earth's Van Allen radiation belts. Nature Communications. 2013, vol. 4, pp. 2795. DOI: 10.1038/ncomms3795.

Mathie R.A., Mann I.R.A correlation between extended intervals of ULF wave power and storm-time geosynchronous relativistic electron flux enhancements. Geophys. Res. Lett. 2000, vol. 27 (20), pp. 3261-3264. DOI: 10.1029/ 2000GL003822.

Mathie R.A., Mann I.R. On the solar wind control of Pc5 ULF pulsation power at mid-latitudes: Implications for $\mathrm{MeV}$ 
electron acceleration in the outer radiation belt. J. Geophys. Res.: Space Phys. 2001, vol. 106 (A12), pp. 29783-29796. DOI: $10.1029 / 2001 \mathrm{JA} 000002$.

Migliuolo S. High- $\beta$ theory of low-frequency magnetic pulsations. J. Geophys. Res. 1983, vol. 88 (A3), pp. 20652074. DOI: 10.1029/JA088iA03p02065.

Mikhailovskii A.B., Pokhotelov O.A. New mechanism for generation of geomagnetic pulsations by fast particles. Soviet J. Plasma Phys. 1975, vol. 1, pp. 786-792.

Mikhailovskii A.B., Pokhotelov O.A. Electromagnetic trapped-electron instability in the magnetosphere. Soviet $J$. Plasma Phys. 1976, vol. 2, pp. 928-935.

Mikhaŭlovskiǐ A.B., Fridman A.M. Drift waves in a finitepressure plasma. Soviet J. Experimental and Theoretical Phys. 1967, vol. 24, pp. 965-974.

Min K., Takahashi K., Ukhorskiy A.Y., Manweiler J.W., Spence H.E., Singer H.J., et al. Second harmonic poloidal waves observed by Van Allen Probes in the dusk-midnight sector. J. Geophys. Res.: Space Phys. 2017, vol. 122 (3), pp. 3013-3039. DOI: 10.1002/2016JA023770.

Moiseev A.V., Baishev D.G., Mullayarov V.A., Samsonov S.N., Uozumi T., Yoshikava A., et al. The development of compression long-period pulsations on the recovery phase of the magnetic storm on May 23, 2007. Cosmic Res. 2016, vol. 54, pp. 31-39. DOI: 10.1134/S0010952516010123.

Moiseev A.V., Starodubtsev S.A., Mishin V.V. Features of excitation and azimuthal and meridional propagation of long-period Pi3 oscillations of the geomagnetic field on December 8, 2017. Solar-Terr. Phys. 2020, vol. 6, pp. 46-59. DOI: $10.12737 /$ stp-63202007.

Motoba T., Takahashi K., Ukhorskiy A., Gkioulidou M., Mitchell D.G., Lanzerotti L.J., et al. Link between premidnight second harmonic poloidal waves and auroral undulations: Conjugate observations with a Van Allen Probe spacecraft and a THEMIS all-sky imager. J. Geophys. Res. 2015, vol. 120, pp. 1814-1831. DOI: 10.1002/2014JA020863.

Ng P.H., Patel V.L. The coupling of shear Alfvén and compressional waves in high- $m$ magnetospheric plasma. $J$. Geophys. Res. 1983, vol. 88 (A12), pp. 10035-10040. DOI: 10.1029/JA088iA12p10035.

Ng P.H., Patel V.L., Chen S. Drift compressional instability in the magnetosphere. J. Geophys. Res. 1984, vol. 89, pp. 10763-10769. DOI: 10.1029/JA089iA12p10763.

Northrop T.G. The Adiabatic Motion of Charged Particles. Interscience, New York, 1963.

O’Brien T.P., Lorentzen K.R., Mann I.R., Meredith N.P., Blake J.B., Fennell J.F., et al. Energization of relativistic electrons in the presence of ULF power and MeV microbursts: Evidence for dual ULF and VLF acceleration. J. Geophys. Res.: Space Phys. 2003, vol. 108 (A8), pp. 1329. DOI: 10.1029/2002JA009784.

Oimatsu S., Nose M., Takahashi K., Yamamoto K., Keika K., Kletzing C. A., et al. Van Allen probes observations of driftbounce resonance and energy transfer between energetic ring current protons and poloidal Pc4 wave. J. Geophys. Res.: Space Phys. 2018, vol. 123 (5), pp. 3421-3435. DOI: 10.1029/2017JA025087.

Ozeke L.G., Mann I.R., Murphy K.R., Rae I.J., Milling D.K., Elkington S. R., et al. ULF wave derived radiation belt radial diffusion coefficients. J. Geophys. Res. 2012, vol. 117, p. A04222. DOI: 10.1029/2011JA017463.

Ozeke L.G., Mann I.R., Murphy K.R., Rae J.I., and Milling D.K. Analytic expressions for ULF wave radiation belt radial diffusion coefficients. J. Geophys. Res.: Space Phys. 2014, vol. 119, pp. 1587-1605. DOI: 10.1002/ 2013JA019204.

Ozeke L.G., Mann I.R., Murphy K.R., Degeling A.W., Claudepierre S.G., Spence H.E. Explaining the apparent impenetrable barrier to ultra-relativistic electrons in the outer
Van Allen belt. Nature Communications. 2018, vol. 9, p. 1844. DOI: 10.1038/s41467-018-04162-3.

Pilipenko V., Kleimenova N., Kozyreva O., Engebretson M., Rasmussen O. Long-period magnetic activity during the may 15, 1997 storm. J. Atmos. Terr. Phys. 2001, vol. 63, pp. 489-501. DOI: 10.1016/S1364-6826(00)00189-9.

Pilipenko V.A., Pokhotelov O.A. Drift-mirror instability in a curved magnetic field. Geomagnetism and Aeronomy. 1977, vol. 17, pp. 161-163.

Pilipenko V.A., Pokhotelov O.A., Feigin F.Z. Influence of bounce resonances on excitation of Alfvén waves beyond the plasmasphere. Geomagnetism and Aeronomy. 1977, vol. 17, pp. 894-899.

Pilipenko V.A., Klimushkin D.Yu., Mager P.N., Engebretson M.J., Kozyreva O.V. Generation of resonant Alfvén waves in the auroral oval. Ann. Geophys. 2016, vol. 34 (2), pp. 241-248. DOI: 10.5194/angeo-34-241-2016.

Pilipenko V.A., Belakhovsky V.B., Samsonov S.N. On a possible acceleration mechanisms of electrons up to the relativistic energies in the Earth magnetosphere. Transactions Kola science centre RAS. 2017, vol. 8, pp. 24-30. (In Russian).

Pokhotelov O.A., Pilipenko V.A. Contribution to the theory of the drift-mirror instability of the magnetospheric plasma. Geomagnetism and Aeronomy. 1976, vol. 16, pp. 296-299.

Pokhotelov O.A., Pilipenko V.A., Amata E. Drift anisotropy instability of a finite-beta magnetospheric plasma. Planet. Space Sci. 1985, vol. 33, pp. 1229-1241. DOI: 10.1016/0032-0633(85)90001-7.

Pokhotelov O.A., Balikhin M.A., Alleyne H.S.-C.K., Onishchenko O.G. Mirror instability with finite electron temperature effects. J. Geophys. Res. 2000, vol. 105, pp. 2393-2402. DOI: 10.1029/1999JA900351.

Pokhotelov O.A., Khabazin Y.G., Mann I.R., Milling D.K., Shukla R.K., and Stenflo L. Giant pulsations: A nonlinear phenomenon. J. Geophys. Res.: Space Phys. 2000, vol. 105 (A5), pp. 10691-10702. DOI: 10.1029/1999JA900506.

Pokhotelov O.A., Balikhin M.A., Sagdeev R.Z., and Treumann R.A. Halo and mirror instabilities in the presence of finite larmor radius effects. J. Geophys. Res.: Space Phys. 2005, vol. 110 (A10), pp. A10206. DOI: 10.1029/ 2004JA010933.

Potapov A.S. ULF wave activity in high-speed streams of the solar wind: Impact on the magnetosphere. J. Geophys. Res.: Space Phys. 2013, vol. 118, pp. 6465-6477. DOI: 10.1002/2013JA019119.

Potapov A. Relativistic electrons of the outer radiation belt and methods of their forecast (Review). Solar-Terr. Phys. 2017, vol. 3 (1), pp. 57-72. DOI: 0.12737/article_ 58f9703837c248.84596315.

Potapov A., Guglielmi A., Tsegmed B., Kultima J. Global Pc5 event during 29-31 October 2003 magnetic storm. Adv. Space Res. 2006, vol. 38 (8), pp. 1582-1586. DOI: 10.1016/j.asr.2006.05.010.

Potapov A.S., Amata E., Polyushkina T.N., Coco I., Ryzhakova L.V. A case study of global ULF pulsations using data from space borne and ground-based magnetometers and a SuperDARN radar. Kosmichna Nauka i Tekhnologia. 2011, vol. 17 (6), pp. 54-67. DOI: 10.15407/knit2011.06.054.

Potapov A.S., Tsegmed B., Ryzhakova L.V. Relationship between the fluxes of relativistic electrons at geosynchronous orbit and the level of ULF activity on the Earth's surface and in the solar wind during the $23^{\text {rd }}$ solar activity cycle. Cosmic Res. 2012, vol. 50 (2), pp. 124-140. DOI: 10.1134/ S0010952512020086.

Potapov A.S., Polyushkina T.N., Pulyaev V.A. Observations of ULF waves in the solar corona and in the solar wind at the Earth's orbit. J. Atmos. Solar-Terr. Phys. 2013, vol. 102, pp. 235-242. DOI: 10.1016/j.jastp.2013.06.001. 
Radoski H.R. Highly asymmetric MHD resonances. The guided poloidal mode. J. Geophys. Res. 1967, vol. 72, pp 4026-4033. DOI: 10.1029/JZ072i015p04026.

Radoski H.R. A theory of latitude dependent geomagnetic micproulsations: the asymptotic fields. J. Geophys. Res. 1974 vol. 79, pp. 595-613. DOI: 10.1029/JA079i004p00595.

Rae I.J., Mann I.R., Watt C.E.J., Kistler L.M., Baumjohann W. Equator-S observations of drift mirror mode waves in the dawnside magnetosphere. J. Geophys. Res. 2007 , vol. 112 (A11), pp. A11203. DOI: 10.1029/2006JA012064.

Rankin R., Wang C.R., Wang Y.F., Zong Q.G., Zhou X.Z., Degeling A.W., et al. Ultra-Low-Frequency WaveParticle Interactions in Earth's Outer Radiation Belt. Geophys. Monograph Ser. 2020, vol. 248, pp. 189-205. Washington: American Geophysical Union Publ., DC, USA, 2020. DOI: 10.1002/9781119509592.ch11.

Ren J., Zong Q.G., Zhou X.Z., Rankin R., Wang Y.F. Interaction of ULF waves with different ion species: Pitch angle and phase space density implications. J. Geophys. Res.: Space Phys. 2016, vol. 121 (10) , pp. 9459-9472. DOI: 10.1002/2016JA022995.2016JA022995.

Ren J., Zong Q.G., Zhou X.Z., Rankin R., Wang Y.F., Gu S.J., and Zhu Y.F. Phase relationship between ULF waves and drift-bounce resonant ions: A statistical study. J. Geophys. Res.: Space Phys. 2017, vol. 122, pp. 7087-7096. DOI 10.1002/2016JA023848. 2016JA023848.

Ren J., Zong Q.G., Zhou X.Z., Spence H.E., Funsten H.O., Wygant J.R., Rankin R. Cold plasmaspheric electrons affected by ULF waves in the inner magnetosphere: A Van Allen Probes statistical study. J. Geophys. Res.: Space Phys. 2019 , vol. 124, pp. 7954-7965. DOI: 10.1029/2019JA027009.

Romanova N., Pilipenko V. ULF wave indices to characterize the solar wind-magnetosphere interaction and relativistic electron dynamics. Acta Geophysica. 2009, vol. 57 (1) , pp. 158-170. DOI: 10.2478/s11600-008-0064-4.

Romanova N., Pilipenko V., Yagova N.V., Belov A.V. Statistical correlation of the rate of failures on geosynchronous satellites with fluxes of energetic electrons and protons. Cosmic Res. 2005, vol. 43 (3), pp. 179-185. DOI: 10.1007/s10604-005-0032-6.

Rostoker G., Skone S., Baker D.N. On the origin of relativistic electrons in the magnetosphere associated with some geomagnetic storms. Geophys. Res. Lett. 1998, vol. 25 (19), pp. 3701-3704. DOI: 10.1029/98GL02801.

Rubtsov A.V., Agapitov O.V., Mager P.N., Klimushkin D.Yu., Mager O.V., Mozer F.S., Angelopoulos V. Drift resonance of compressional ULF waves and substorm-injected protons from multipoint THEMIS measurements. J. Geophys. Res.: Space Phys. 2018, vol. 123 (11) , pp. 9406-9419. DOI: 10.1029/2018JA025985.

Rubtsov A.V., Mikhailova O.S., Mager P.N., et al. Multispacecraft observation of the presubstorm long-lasting poloidal ULF wave. Geophys. Res. Lett. 2021, vol. 48, iss. 23. e2021GL096182. DOI: 10.1029/2021GL096182.

Saka O., Iijima T., Yamagishi H., Sato N., Baker D.N. Excitation of Pc-5 pulsations in the morning sector by a local injection of particles in the magnetosphere. J. Geophys. Res. 1992, vol. 97, pp. 10,693-10,701. DOI: 10.1029/92JA00441.

Saka O., Watanabe O., Baker D.N. A possible driving source for transient field line oscillations in the postmidnight sector at geosynchronous altitudes. J. Geophys. Res. 1996, vol 101, pp. 24,719-24,726. DOI: 10.1029/96JA02039.

Sandhu J.K., Rae I.J., Wygant J.R., Breneman A.W., Tian S., Watt C.E.J., et al. ULF wave driven radial diffusion during geomagnetic storms: A statistical analysis of Van Allen Probes observations. J. Geophys. Res.: Space Phys. 2021, vol 126 (4), p. e2020JA029024. DOI: 10.1029/2020JA029024.

Sarris T.E., Wright A.N., Li X. Observations and analysis of Alfvén wave phase mixing in the Earth's magnetosphere. $J$.
Geophys. Res.: Space Phys. 2009, vol. 114, p. A03218. DOI: 10.1029/2008JA013606.

Schulz M., Lanzerotti L.J. Particle diffusion in the radiation belts. Springer-Verlag, Berlin, Heidelberg, New York, 1974. 218 p.

Shprits Y.Y., Elkington S.R., Meredith N.P., Subbotin D.A. Review of modeling of losses and sources of relativistic electrons in the outer radiation belt. I: Radial transport. $J$. Atmos. Solar-Terr. Phys. 2008, vol. 70 (14) , pp. 1679-1693. DOI: $10.1016 / j . j a s t p .2008 .06 .008$.

Simms L.E., Engebretson M.J., Rodger C.J., Dimitrakoudis S., Mann I.R., Chi P.J. The combined influence of lower band chorus and ULF waves on radiation belt electron fluxes at individual 1-shells. J. Geophys. Res.: Space Phys. 2021, vol. 126 (5), p. e2020JA028755. DOI: 10.1029/2020JA028755.

Southwood D.J. Preservation of the second adiabatic invariant during cross- L diffusion. J. Geophys. Res. 1972, vol. 77 (7) , pp. 1123-1127. DOI: 10.1029/JA077i007p01123.

Southwood D.J. The behaviour of ULF waves and particles in the magnetosphere. Planet. Space Sci. 1973, vol. 21, pp. 53-65. DOI: 10.1016/0032-0633(73)90019-6.

Southwood D.J. Some features of field line resonances in the magnetosphere. Planet. Space Sci. 1974, vol. 22, pp. 483491. DOI: 10.1016/0032-0633(74)90078-6.

Southwood D.J. A general approach to low-frequency instability in the ring current plasma. J. Geophys. Res.: Space Phys. 1976, vol. 81, pp. 3340-3348. DOI: 10.1029/JA081i019p03340.

Southwood D.J. Low frequency pulsation generation by energetic particles. J. Geomagn. Geoelectricity. 1980, Suppl. II, vol. 32, pp. 75-88.

Southwood D.J., Kivelson M.G. Charged particle behavior in low-frequency geomagnetic pulsations. 1. Transverse waves. J. Geophys. Res. 1981, vol. 86 (A7), pp. 5643-5655. DOI: $10.1029 /$ JA086iA07p05643.

Southwood D.J., Kivelson.M.G. Charged particle behavior in low-frequency geomagnetic pulsations. 2. Graphical approach. J. Geophys. Res. 1982, vol. 87, pp. 1707-1710. DOI: 10.1029/JA087iA03p01707.

Southwood D. J., Dungey J. W., Etherington R. J. Bounce resonant interactions between pulsations and trapped particles. Planet. Space Sci. 1969, vol. 17, pp. 349-361. DOI: 10.1016/0032-0633(69)90068-3.

Su S.Y., Konradi A., Fritz T.A. On propagation direction of ring current proton ULF waves observed by ATS 6 at 6.6R $R_{\mathrm{E}}$. J. Geophys. Res. 1977, vol. 82(13), pp. 1859-1868. DOI: $10.1029 / J A 082 \mathrm{i} 013$ p01859.

Su Z., Zhu H., Xiao F., Zong Q. G., Zhou X. Z., Zheng H., et al. Ultra-low-frequency wave-driven diffusion of radiation belt relativistic electrons. Nature Communications. 2015, vol. 6, p. 10096. DOI: 10.1038/ncomms10096.

Tajiri M. Propagation of hydromagnetic waves in collisionless plasma. II. Kinetic approach. J. Physical Society of Japan. 1967, vol. 22 (6), pp. 1482-1494. DOI: 10.1143/JPSJ.22.1482.

Takahashi K. New observations, new theoretical results and controversies regarding Pc3-5 waves. Adv. Space Res. 1996, vol. 17 (10), pp. 63-71. DOI: 10.1016/02731177(95)00696-C

Takahashi K., Fennell J.F., Amata E., and Higbie P.R. Field-aligned structure of the storm time Pc5 wave of November 14-15, 1979. J. Geophys. Res.: Space Phys. 1987, vol. 92 (A6), pp. 5857-5864. DOI: 10.1029/JA092iA06p05857.

Takahashi K., McEntire R.W., Lui A.T.Y., Potemra T.A. Ion flux oscillations associated with a radially polarized transverse Pc5 magnetic pulsation. J. Geophys. Res. 1990, vol. 95, pp. 3717-3731. DOI: 10.1029/JA095iA04p03717. 
Takahashi K., Claudepierre S.G., Rankin R., Mann I., Smith C.W. Van Allen Probes Observation of a Fundamental Poloidal Standing Alfvén wave event related to giant pulsations. J. Geophys. Res.: Space Phys. 2018a, vol. 123, pp. 4574-4593. DOI: 10.1029/2017JA025139.

Takahashi K., Oimatsu S., Nose M., Min K., Claudepierre S.G., Chan A., et al. Van Allen Probes observations of second harmonic poloidal standing Alfvén waves. J. Geophys. Res.: Space Phys. 2018b, vol. 123, pp. 611-637. DOI: 10.1002/2017JA024869.

Tamao T. Interaction of energetic particles with HMwaves in the magnetosphere. Planet. Space Sci. 1984a, vol. 32, pp. 1371-1386. DOI: 10.1016/0032-0633(84)90080-1.

Tamao T. Magnetosphere - ionosphere interaction through hydromagnetic waves. Achievements of the International Magnetospheric Study (IMS). ESA Special Publ. 1984b, vol. 217, pp. 427-435.

Tian A., Xiao K., Degeling A.W., Shi Q., Park J.-S., Nowada M., Pitkänen T. Reconstruction of plasma structure with anisotropic pressure: Application to Pc5 compressional wave. Astrophys. J. 2020, vol. 889 (1), pp. 35. DOI: 10.3847/1538-4357/ab6296.

Trakhtengerts V.Y., Rycroft M.J. Whistler and Alfvén Mode Cyclotron Masers in Space. Cambridge University Press, 2008. 354 p.

Tsurutani B.T., Lakhina G.S. Some basic concepts of wave-particle interactions in collisionless plasmas. Rev. Geophys. 1997, vol. 35 (4), pp. 491-501. DOI: 10.1029/ 97RG02200.

Ukhorskiy A., Sitnov M.I. Radial transport in the outer radiation belt due to global magnetospheric compressions. $J$ Atmos. Solar-Terr. Phys. 2008, vol. 70 (14) , pp. 1714-1726. DOI: 10.1016/j.jastp.2008.07.018.

Ukhorskiy A.Y., Anderson B.J., Takahashi K., Tsyganenko N.A. Impact of ULF oscillations in solar wind dynamic pressure on the outer radiation belt electrons. Geophys. Res. Lett. 2006, vol. 33 (6), pp. L06111. DOI: 10.1029/2005GL024380.

Ukhorskiy A.Y., Sitnov M.I., Takahashi K., Anderson B.J. Radial transport of radiation belt electrons due to stormtime Pc5 waves. Ann. Geophys. 2009, vol. 27, pp. 2173-2181. DOI: 10.5194/angeo-27-2173-2009.

Vaivads A., Baumjohann W., Georgescu E., Haerendel G., Nakamura R., Lessard M. R., et al. Correlation studies of compressional Pc5 pulsations in space and Ps6 pulsations on the ground. J. Geophys. Res.: Space Phys. 2001, vol. 106 (A12), pp. 29797-29806. DOI: 10.1029/2001JA900042.

Vetoulis G., Chen L. Global structures of Alfvénballooning modes in magnetospheric plasmas. Geophys. Res. Lett. 1994, vol. 21, pp. 2091-2094. DOI: 10.1029/94GL01703.

Walker A. D. M., Greenwald R. A., Korth A., Kremser G. STARE and GEOS-2 observations of a storm time Pc5 ULF pulsation. J. Geophys. Res. 1982, vol. 87, pp. 9135-9146. DOI: 10.1029/JA087iA11p09135.

Wang B., Zhang H., Liu Z., Liu T., Li X., Angelopoulos $\mathrm{V}$. Energy modulations of magnetospheric ions induced by foreshock transient-driven ultralow-frequency waves. Geophys. Res. Lett. 2021, vol. 48 (10), e2021GL093913. DOI: 10.1029/2021GL093913.

Wang C., Rankin R., Zong Q. Fast damping of ultralow frequency waves excited by interplanetary shocks in the magnetosphere. J. Geophys. Res.: Space Phys. 2015, vol. 120 , pp. 2438-2451. DOI: 10.1002/2014JA020761.

Watson C., Jayachandran P.T., Singer H.J., Redmon R.J., Danskin D. GPS TEC response to Pc4 "giant pulsations". $J$. Geophys. Res.: Space Phys. 2016, vol. 121 (2), pp. 17221735. DOI: $10.1002 / 2015$ JA022253.

Wei C., Dai L., Duan S.-P., Wang C., Wang Y.-X.
Multiple satellites observation evidence: High- $m$ poloidal ULF waves with time-varying polarization states. Earth and Planetary Phys. 2019, vol. 3 (3), pp. 190-203. DOI: 10.26464/epp2019021.

Woch J., Kremser G., Korth A., Pokhotelov O.A., Pilipenko V.A. Curvature-driven drift mirror instability in the magnetosphere. Planet. Space Sci. 1988, vol. 36, pp. 383-393. DOI: 10.1016/0032-0633(88)90126-2.

Woch J., Kremser G., Korth A. A comprehensive investigation of compressional ULF waves observed in the ring current. J. Geophys. Res. 1990, vol. 95, pp. 15113-15132. DOI: 10.1029/JA095iA09p15113.

Wright D.M., Yeoman T.K., Rae I.J., Storey J., StocktonChalk A.B., Roeder J.L., Trattner K.J. Ground-based and Polar spacecraft observations of a giant $(\mathrm{Pg})$ pulsation and its associated source mechanism. J. Geophys. Res. 2001, vol. 106, pp. 10837-10852. DOI: 10.1029/2001JA900022.

Xia Z., Chen L., Zheng L., Chan A.A. Eigenmode analysis of compressional poloidal modes in a selfconsistent magnetic field. J. Geophys. Res.: Space Phys. 2017, vol. 122 (A11), pp. 10369-10381. DOI: 10.1002/2017JA024376.

Yagova N.V., Pilipenko V.A., Sakharov Y.A., Selivanov V.N. Spatial scale of geomagnetic Pc5/Pi3 pulsations as a factor of their efficiency in generation of geomagnetically induced currents. Earth, Planets and Space. 2021, vol. 73 (1), pp. 88. DOI: 10.1186/s40623-021-01407-2.

Yamakawa T., Seki K., Amano T., Takahashi N., Miyoshi Y. Excitation of internally driven ULF waves by the drift-bounce resonance with ring current ions based on the drift-kinetic simulation. J. Geophys. Res.: Space Phys. 2020, vol. 125 (11), p. e28231. DOI: 10.1029/2020JA028231.

Yamamoto K., Nose M., Keika K., Hartley D.P., Smith C.W., MacDowall R.J., et al. Eastward propagating second harmonic poloidal waves triggered by temporary outward gradient of proton phase space density: Van Allen Probe A observation. J. Geophys. Res.: Space Phys. 2019, vol. 124 (12), pp. 9904-9923. DOI: 10.1029/2019JA027158.

Yang B., Zong Q.-G., Fu S.Y., Li X., Korth A., Fu H.S., et al. The role of ULF waves interacting with oxygen ions at the outer ring current during storm times. J. Geophys. Res.: Space Phys. 2011, vol. 116 (A1), p. A01203. DOI: 10.1029/2010JA015683.

Yeoman T. K., Wright D. M., Chapman P.J., StocktonChalk A.B. High-latitude observations of ULF waves with large azimuthal wave numbers. J. Geophys. Res. 2000, vol. 105, pp. 5453-5462. DOI: 10.1029/1999JA005081.

Yeoman T.K., Klimushkin D.Yu., Mager P.N. Intermediate- $m$ ULF waves generated by substorm injection: a case study. Ann. Geophys. 2010, vol. 28, pp. 1499-1509. DOI: 10.5194/angeo-28-1499-2010.

Yeoman T.K., James M., Mager P.N., Klimushkin D.Yu. SuperDARN observations of high-m ULF waves with curved phase fronts and their interpretation in terms of transverse resonator theory. J. Geophys. Res. 2012, vol. 117, p. A06231. DOI: 10.1029/2012JA017668.

Zelenyi L.M., Veselovsky I.S. Plasma heliogeophysics. Vol. 2. Moscow, Fizmatlit, 2010. (In Russian). 560 p.

Zhai C., Shi X., Wang W., Hartinger M.D., Yao Y., Peng W., et al. Characterization of high-m ULF wave signatures in GPS TEC data. Geophys. Res. Lett. 2021, vol. 48 (14), p. e2021GL094282. DOI: 10.1029/2021GL094282.

Zhou X.-Z., Wang Z.-H., Zong Q.-G., Rankin R., Kivelson M.G., Chen X.-R., et al. Charged particle behavior in the growth and damping stages of ultralow frequency waves: Theory and Van Allen Probes observations. J. Geophys. Res.: Space Phys. 2016, vol. 121 (4), pp. 3254-3263. DOI: 10.1002/2016JA022447.

Zolotukhina N.A. On excitation of Alfvén waves in the magnetosphere by a moving source. Issledovaniya po 
geomagnetizmu, aeronomii $i$ fizike Solntsa [Research on Geomagnetism, Aeronomy and Solar Physics]. Nauka Publ., 1974, iss. 34, pp. 20-23. (In Russian).

Zolotukhina N.A., Mager P.N., Klimushkin D.Yu. Pc5 waves generated by substorm injection: a case study. Ann. Geophys. 2008, vol. 26, pp. 2053-2059. DOI: 10.5194/angeo26-2053-2008.

Zong Q.-G., Zhou X.-Z., Wang Y. F., Li X., Song P., Baker D. N., et al. Energetic electron response to ULF waves induced by interplanetary shocks in the outer radiation belt. $J$. Geophys. Res. 2009, vol. 114 (A10), pp. A10204. DOI: 10.1029/2009JA014393.

Zong Q.-G., Yuan C. J., Yang B., Wang C.R., Zhang X.Y. Fast acceleration of "killer" electrons and energetic ions by interplanetary shock stimulated ULF waves in the inner magnetosphere. Chinese Sci. Bull. 2011, vol. 56 (12), p. 1188. DOI: $10.1007 / \mathrm{s} 11434-010-4308-8$.

Zong Q.-G., Wang Y. F., Zhang H., Fu S. Y., Zhang H., Wang C. R., et al. Fast acceleration of inner magnetospheric hydrogen and oxygen ions by shock induced ULF waves. $J$. Geophys. Res. 2012, vol. 117 (A11), p. A11206. DOI: 10.1029/2012JA018024.

Zong Q.-G., Rankin R., Zhou X. The interaction of ultralow-frequency Pc3-5 waves with charged particles in Earth's magnetosphere. Rev. Modern Plasma Phys. 2017, vol. 1 (1), p. 10. DOI: $10.1007 / \mathrm{s} 41614-017-0011-4$.

Zong Q.-G., Leonovich A. S., Kozlov D. A. Resonant Alfvén waves excited by plasma tube/shock front interaction. Physics of Plasmas, vol. 25 (12), p. 122904, 2018. DOI: 10.1063/1.5063508.

\section{How to cite this article}

Klimushkin D.Yu., Mager P.N., Chelpanov M.A., Kostarev D.V. Interaction of the long-period ULF waves and charged particle in the magnetosphere: theory and observations (overview). Solar-Terrestrial Physics. 2021. Vol. 7. Iss. 4. P. 33-66. DOI: 10.12737/stp-74202105. 